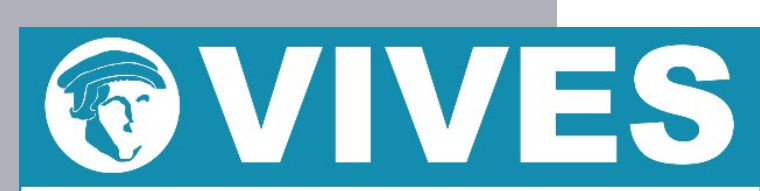

RESEARCH CENTER FOR REGIONAL ECONOMICS

\title{
Do Prices Rise faster than they Fall? Evidence from Scanner Data
}

\section{Kim In Kyung}

Nazarbayev University, Department of Economics

\section{Joep Konings}

KU Leuven VIVES, University of Liverpool Management School, Nazarbayev University and CEPR

\section{Lee Jinhyuk}

Korea University, Department of Economics 


\title{
Do prices rise faster than they fall? Evidence from scanner data*
}

\author{
In Kyung Kim ${ }^{\dagger} \quad$ Jozef Konings ${ }^{\ddagger} \quad$ Jinhyuk Lee ${ }^{\S}$
}

December 2020

\begin{abstract}
A stylized fact is that prices of goods tend to react and rise faster in response to an increase in costs of intermediate inputs, than they do when the costs of intermediate inputs decrease. This paper uses scanner data of retailers in an emerging economy, Kazakhstan, to analyze how large exchange rate shocks have an impact on consumer prices. We find, as in most of the literature, incomplete exchange rate pass through (ERPT) into consumer prices, even after 12 months of the initial shock. This ERPT, however, is heterogeneous and depends on the type of the product (imported versus domestic), the size of the shock, and the size of the retailer. In particular, ERPT is higher for imported products $(50 \%)$ than for domestic products (25\%); ERPT is also non-linear and higher for large retailers. Distinguishing the impacts of appreciation and depreciation reveals that ERPT is asymmetric and the direction of asymmetry is the opposite between imported and domestic products. This finding suggests that domestic producers may be keen on preserving the market share while foreign manufacturers may be more concerned with increasing markup.
\end{abstract}

\section{Keywords: exchange rate pass through, consumer prices, scanner data, inflation dynamics}

\section{JEL Classification Numbers: F37, L16, F1}

${ }^{*}$ We thank Dameli Baltabekova and Madina Kazhen for excellent research assistance, and other 58 students at Nazarbayev University for manual data collection. We are also grateful to seminar participants at Nazarbayev University. Financial support from the Seed Program for Korean Studies through the Ministry of Education of the Republic of Korea (AKS-2018-INC-2230011) and the Small Grant Program at Nazarbayev University (SHSS2018004) is gratefully acknowledged. The authors declare that they have no conflict of interest.

${ }^{\dagger}$ Department of Economics, Nazarbayev University, E-mail: in.kim@nu.edu.kz.

${ }^{\ddagger}$ GSB, Nazarbayev University and CEPR; ULMS; KU Leuven, E-mail: joep.konings@kuleuven. be

$\S$ Department of Economics, Korea University, E-mail: jinhyuklee@korea.ac.kr . 


\section{Introduction}

A stylized fact is that prices of goods tend to react and rise faster in response to an increase in costs of intermediate input costs, than they do when the costs of intermediate inputs decrease. This fact has been well documented in advanced economies, like the U.S., for industrial producer prices, agricultural prices and consumer prices (e.g. Peltzman (2000)). The degree of such asymmetry seems to be related to the complexity of the supply chain. For instance, when such a cost shock is filtered through a fragmented wholesale distribution system, the asymmetric response is larger. In contrast, when an individual decision maker like a single supermarket chain controls the pricing decision, less or even no asymmetry is found.

An important source of such cost shocks is the volatility of exchange rates as they play a key role in the global transmission of shocks via trade linkages. A vast literature has shown that exchange rate pass through (ERPT) into prices is incomplete, that is, a depreciation only results in a limited increase in prices. ${ }^{1}$ Most of the focus of the literature has been on explaining the 'disconnect' between exchange rates and prices, assuming exchange rate depreciations and appreciations pass on symmetrically. Furthermore, no distinction is made between small and large exchange rate shocks. Recent work for the U.S., however, has shown asymmetric responses to exchange rate shocks in BLS import prices (Kim, Lewis, and Vigfusson, 2018). The asymmetric nature of ERPT and potentially non-linear responses, may be even more relevant when there are large exchange rate fluctuations, which are more prevalent in emerging economies. Hence, this is particularly relevant for understanding the transmission mechanisms of monetary policy. The level and speed of price adjustment in response to international shocks matters for fine-turning monetary policy when inflation targeting is important. Knowing how ERPT takes place is an important component of monetary policy in most countries. When the response of consumer prices, which constitute an important part of core inflation, is stronger after a depreciation than after an appreciation, the level of monetary easing or restriction matters to reach a desired inflation target.

This paper analyses the asymmetric nature of ERPT in an emerging economy, Kazakhstan. To

\footnotetext{
${ }^{1}$ Various channels can explain this exchange rate disconnect: Gopinath and Rigobon (2008) focus on the role of local currency pricing; pricing to market (PTM) happens when firms choose the markup and optimally chose different prices depending on local market conditions as in Atkeson and Burstein (2008); Goldberg and Campa (2010) argue that local distribution costs are causing a wedge between consumer prices and import prices, and finally Amiti, Itskhoki, and Konings (2014) show that the largest exporters are also the largest importers and hence firms have a hedging mechanism allowing them to keep prices in destination markets stable when exchange rate shocks occur.
} 
this end, we use detailed scanner data on products sold in retailers (mostly supermarkets) covering the period from January 2014 to December 2016. This is particularly interesting as there has been no granular work on asymmetric responses in emerging economies, which typically experience larger exchange rate fluctuations. Furthermore, competition in markets is not comparable to competition in well- established market economies. Hence, not much is known whether such asymmetric responses are also prevalent in emerging market economies. Second, Kazakhstan is a particularly interesting case as it has experienced large exchange rate shocks, first with the devaluation in 2014 and second with the switch from a fixed exchange rate to a floating exchange rate system in August 2015. There have also been periods of an appreciation of the currency, the Tenge, which allows an analysis of asymmetric responses.

We make three contributions. First, we combine scanner data on consumer prices with a large unanticipated exchange rate shock to study exchange rate pass-through. Hence, we do not have to rely on aggregate consumer price indices, such as in Goldberg and Campa (2010), or on small exchange rate movements for which the underlying sources are unknown, like in Antoniades and Zaniboni (2016). Second, we use local projections to be able to analyze the impact of the exchange rate shock over time while accounting for the dynamics in the exchange rate after the shock, which the standard distributed lag models are not able to do. Third, we provide new evidence on the magnitude and speed of ERPT into consumer prices in an emerging economy, which typically experience more volatile exchange rate and higher inflation than developed economies. Furthermore, we document the extent of asymmetric responses of ERPT and show how these depend on the nature of the products (locally sourced or foreign sourced).

Our main findings are the following: While ERPT is incomplete, it is lower for domestically sourced products (around 25 percent) than for foreign sourced products (around 50 percent). We also find that while ERPT is asymmetric, the nature of the asymmetry is different and opposite for domestically sourced products than for foreign sourced ones. More specifically, the piecewise linear price reaction function is convex for imported products, but concave for domestic products. This finding suggests that domestic producers may be keen on preserving the market share, while foreign producers may be more concerned with increasing markup (Bussiere, 2013)). We reach the same conclusion when we differentiate between small and large exchange rate shocks. Estimation results also show large retailers tend to have larger ERPT compared to small/medium size retailers, 
suggesting that larger retailers may have lower local costs or lower markups, and therefore rely more on the exchange rate (Corsetti and Dedola, 2005; Hellerstein, 2008; Antoniades and Zaniboni, 2016).

We proceed as follows. In the next section, we describe recent exchange rate fluctuations in Kazakhstan, and introduce the data used for our empirical analysis. Section 3 discusses the empirical results, and Section 4 concludes the paper.

\section{Background and data}

\section{Exchange rate shocks}

Kazakhstan used to maintain a pegged exchange rate, tying its currency, Tenge, to a multi-currency basket of its three trading partners: US Dollar (70 percent), Chinese Yuan (20 percent), and Russian Ruble (10 percent). Facing an increase in the balance of payments deficit, on 11 February 2014 the National Bank of Kazakhstan declared to depreciate Tenge by almost 20 percent, and raised the exchange rate from around 155 Tenge per dollar to around 185 Tenge. The top left panel of Figure 1 shows that the exchange rates between Tenge and currencies of its other major trading partners such as EU also rose during the first half of 2014.

Meanwhile, according to the top right panel of Figure 1, the price of crude oil plunged by more than 40 percent in the middle of 2014. The rapid decline of oil prices due to the global economic recession led to the economic downturn of the Russian Federation (Russia, hereafter) as energy resources such as crude oil, petroleum, and natural gas account for almost 70 percent of its commodity exports. Sanctions that had been taken since March 2014 against Russia after its annexation of Crimea further aggravated its economy. Examples of sanctions imposed by EU and the US include asset freezing for persons and entities responsible for the crisis, ban on loans for major state-owned banks, and ban on provision of financial and insurance services to energy companies. The bottom panel of Figure 1 demonstrates that as a result, the real GDP growth of Russia had been below zero for seven consecutive quarters between 2014 and $2016 .{ }^{2}$ The economic slowdown of Russia resulted in a sharp depreciation of Ruble against US dollar, and it also kept weakened against Tenge throughout the second half of 2014, as can be seen in the top left panel of Figure 1.

\footnotetext{
${ }^{2}$ Real GDP growth is measured as real GDP relative to real GDP at the same quarter of the previous year.
} 
Figure 1: Trends

(a) Exchange rate

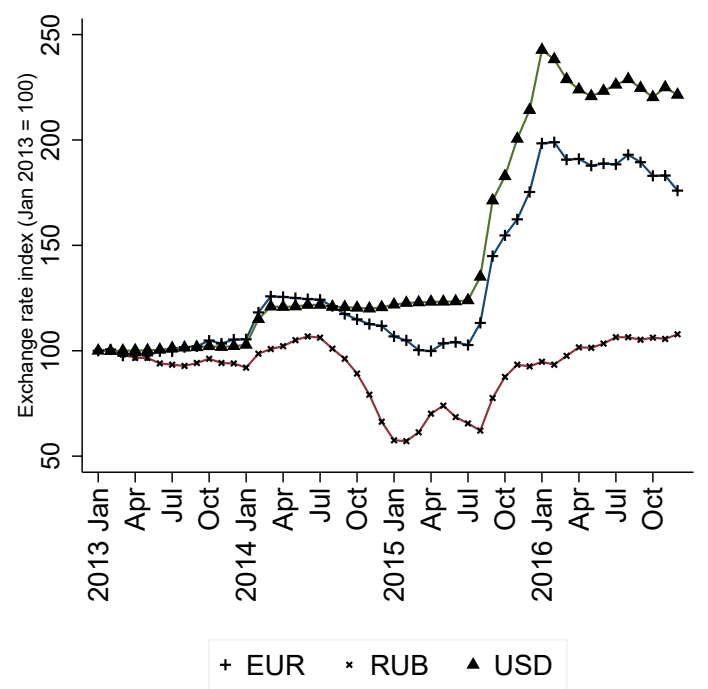

(b) Crude oil price

(c) Real GDP growth

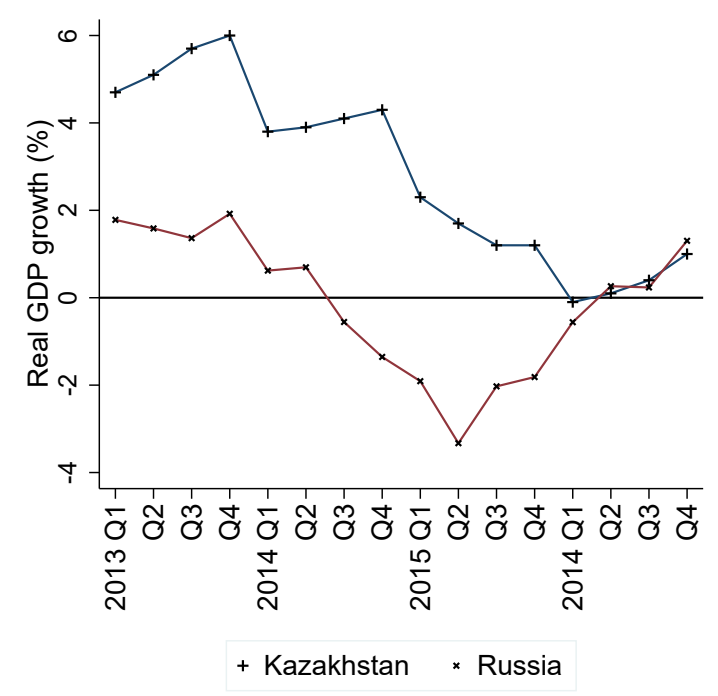

Note: Real GDP growth is measured as real GDP relative to real GDP at the same quarter of the previous year. Source: The Federal Reserve Bank of St. Louis and the National Bank of Kazakhstan

Like Russia, the economy of Kazakhstan was also vulnerable to the falling price of natural resources, its main export products. The bottom panel of Figure 1, for instance, shows that the real GDP growth of Kazakhstan in the second quarter declined from 5.1 percent in 2013 via 3.9 percent in 2016 to 1.7 percent in 2015. The sharp depreciation of Ruble described above imposed additional burden on the economy, especially on the supply side; domestic producers either had to cut the 
Table 1: Production countries

\begin{tabular}{lrrrr}
\hline \hline & \multicolumn{2}{c}{ Products } & \multicolumn{2}{c}{ Sales revenue } \\
\cline { 2 - 5 } Country & $\#$ & $\%$ & in Mil. Tenge & $\%$ \\
\hline & & & & \\
Domestic & & & & \\
Kazakhstan & 899 & 61.6 & $1,025,874$ & 80.2 \\
& & & & \\
Imported & & & & \\
Russia & 414 & 28.4 & 205,817 & 16.1 \\
Georgia & 6 & 0.4 & 14,961 & 1.2 \\
China & 31 & 2.1 & 12,849 & 1.0 \\
Germany & 38 & 2.6 & 6,262 & 0.5 \\
Belarus & 22 & 1.5 & 5,484 & 0.4 \\
Kyrgyzstan & 8 & 0.5 & 4,793 & 0.4 \\
Others & 41 & 2.8 & 2,840 & 0.2 \\
& & & & \\
Total & 1,459 & 100.0 & $1,278,880$ & 100.0 \\
& & & & \\
\hline \hline
\end{tabular}

price sufficiently or lost consumers to their competitors from Russia, the main trading partner of Kazakhstan. Consequently, on 19 August 2015 the National Bank of Kazakhstan announced the transition to freely floating exchange rate system, which was followed by a huge depreciation of Tenge over the second half of 2015, as the top left panel of Figure 1 demonstrates.

\section{Data}

The data come from Nielsen Kazakstan Retail Measurement Service and cover fast-moving consumer goods (FMCGs) in 23 product categories from January 2014 to December 2016. For each product in the data, we collect the production country information by manually checking the package or by contacting the producer or distributor of the product. ${ }^{3}$ After dropping 36 products whose production country could not be collected, ${ }^{4}$ the sample consists of 138,664 product-retailer category-month observations of sales figures as well as retail prices. It is unbalanced due to the introduction of new products and discontinuation of existing products. We complement the data with (i) monthly nominal exchange rate information available at the National Bank of Kazakhstan and (ii) monthly consumer price index for each production country obtained from the IMF.

Table 1 describes that while 60 percent of the 1,459 products in the sample are locally produced,

\footnotetext{
${ }^{3}$ Production country can be different from the product's country of origin. For example, an international brand typically operates regional offices and factories which produce and supply its products to their regions.

${ }^{4}$ The combined total sales of the excluded 36 products is less than 1 percent.
} 
majority of the imported products (414 out of 596) are from Russia, accounting for 16 percent of the total sales revenue. The large presence of Russian products in the data together with a large scale depreciation of Ruble against Tenge in 2014 followed by reversion of its value in the next two years provides an ideal environment to test the asymmetry of ERPT. This point can be also seen in Figure 2 which shows that the monthly exchange rate change is widely dispersed in the data. There are cases where the local currency lost 20 percent of its value or more against a foreign currency, whereas sometimes it gained 20 percent or more. The variation in the exchange rate is much larger than those exploited in the previous works (Antoniades and Zaniboni, 2016; Kim, Lewis, and Vigfusson, 2018).

In Table A1 in the appendix, we compare average unit prices of the two product groups, domestic and imported products, in each of the 23 product categories. ${ }^{5}$ In 17 out of 20 categories where products of both groups are observed, the average price is higher by $6-125$ percent for imported products compared to domestic ones. This suggests that in general imported products may have higher quality than domestic counterparts.

Figure A1 in the appendix presents price trends of local products and products imported from Russia in the 19 product categories, where Russian products are observed. According to the figure, the average unit prices of products imported from Russia tend to be higher than the prices of local products in most product categories. Also, the price responses of Russian products seem faster and greater to the depreciation of Tenge against Ruble that took place in the third quarter of 2015 than to the appreciation that occurred in the third quarter of the previous year. The average unit price jumps or plunge in several product categories such as 'Cheese curds' and 'Soft drinks'. Such abnormal price changes may be due to the change in the product composition as well as the exchange rate shock. Indeed, we obtain smoother price trends using only those products that are observed during the entire sample period, as Figure A2 in the appendix demonstrates. This observation motivates us to consider a balanced panel of products in the empirical analysis, and check the robustness of the empirical findings using the unbalanced panel.

\footnotetext{
${ }^{5}$ Unit price is the price per kilo or liter.
} 
Figure 2: Distribution of exchange rate change

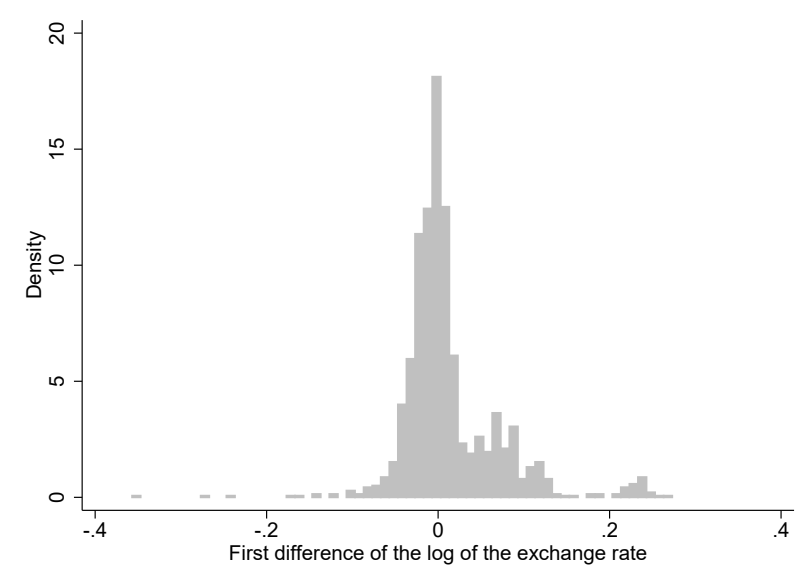

\section{$3 \quad$ Empirical analysis}

In this section, we measure the ERPTs by implementing the local projection method developed by Jordà (2005) on our panel data. We first conduct the analysis separately for imported products and domestic products. Then, leveraging the large scale swings in the exchange rates during the sample period, we further scrutinize the differential effects of appreciation and depreciation on the retail prices of imported and domestic products. We also examine whether the size of the exchange rate pass-though depends on the retailer size, and check the robustness of the empirical findings.

\subsection{Estimating the ERPTs for imported and domestic products}

We begin our analysis by estimating the ERPTs on the retail price of imported products. Our observations are for item (product-retailer category) $i$ at time (year-month) $t$ in the following specification:

$$
\Delta_{h} \ln p_{i, t+h}=\alpha_{i}^{h}+\beta^{h} \Delta \ln e r_{i, t}+\sum_{l=1}^{L} \gamma_{l}^{h} \Delta \ln e r_{i, t-l}+\sum_{l=1}^{L} \delta_{l}^{h} \Delta \ln p_{i, t-l}+\sum_{l=1}^{L} \lambda_{l}^{h} \mathbf{x}_{i, t-l}+\varepsilon_{i, t+h}
$$

where the dependent variable $\Delta_{h} \ln p_{i, t+h}$ is the change in the log price of imported item $i$ between $t-1$ and $t+h, h=0, \cdots, 12 . \alpha_{i}^{h}$ is the item fixed effect, and $\Delta \ln e r_{i, t}$ is the first difference of the $\log$ of the exchange rate between Tenge and the currency of the country that exports item $i$. Hence, its coefficient $\beta^{h}$ measures the price response at horizon $h$ to the exchange rate shock. Given that the price may not only be serially correlated, but also gradually adjust to the exchange rate shock, 
Figure 3: ERPT - imported products

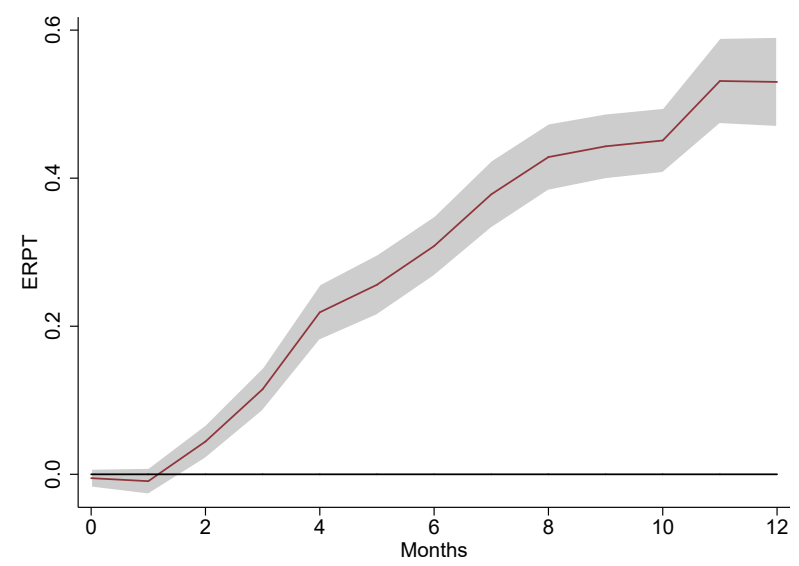

Note: The figure graphically present the estimates of the ERPTs for imported products up to $h=12$, that is, up to one year after the shock, for $L=5$ along with 95 percent confidence bands.

we include lagged first differences of the log exchange rate and the log price as regressors in the model. The vector $\mathbf{x}$ includes the first differences of the log of other control variables that may affect the retail price. We consider two such variables; (i) quantity sold, $q$, and (ii) consumer price index $(C P I)$ of the country that exports the item. The former controls for shifts in the retail price driven by changes in the demand condition, while the latter captures shifts in the marginal cost of the item. The model includes $L$ lags of the vector. ${ }^{6}$ Following previous literature (Klenow and Kryvtsov, 2008; Colicev, Hoste, and Konings, 2019), we consider three values of $L: 4,5$, and 6 . We correct standard errors for product-level clusters, allowing for arbitrary heteroskadasticity and any kind of correlation within a product.

Figure 3 graphically present the estimates of the ERPTs up to $h=12$, that is, up to one year after the shock, for $L=5$ along with 95 percent confidence bands. ${ }^{7}$ The results indicate that the retail price of an imported product does not respond instantaneously to the exchange rate shock, and the ERPT becomes positive and statistically significant only one or two months after the shock. Since then, the pass-through rises constantly and reaches 50 percent one year after the shock. ERPTs when including four and six lags of the control variables are presented in the top panel of Figure A4 in the appendix, and show a similar trend.

\footnotetext{
${ }^{6}$ In case of $h=0$, the dependent variable, $\ln p_{t}-\ln p_{t-1}$, would be correlated with the lagged variable $\ln q_{t-1}-$ $\ln q_{t-2}$. Therefore, for $h=0$ we include $\Delta \ln q_{i, t-l}$ for $l$ from 2 to $L$.

${ }^{7}$ The full estimation results of model (1) for $h=0,2, \cdots, 10,12$ and $L=5$ are reported in Table A2 in the appendix.
} 
Exchange rate shock can both directly and indirectly affect the retail price of domestically produced goods. The direct effect would be through the change in the price of imported ingredients or inputs. Also, as the price of competing imported goods in the same product category are affected by the exchange rate shock, there is also a strategic incentive for local producers to adjust prices of their products.

To quantify the effect of exchange rate change on the price of domestic products, we first construct a sales-weighted change in the exchange rate for a given product category and time:

$$
\ln \left(\frac{e r_{t}^{*}}{e r_{t-1}^{*}}\right)=\sum_{c=1}^{C} w_{c, t} \ln \left(\frac{e r_{c, t}}{e r_{c, t-1}}\right)
$$

or equivalently

$$
\Delta \ln e r_{t}^{*}=\sum_{c=1}^{C} w_{c, t} \Delta \ln e r_{c, t}
$$

where the set $\{1, \cdots, C\}$ includes all exporting countries in the product category. The weight $w_{c, t}$ for the exporting country $c$ at time $t$ is the sales (number of packages sold) proportion of the products imported from country $c$ among all imported products in the product category at time $t .^{8,9}$ Each panel of Figure A3 in the appendix presents the trend of the sales-weighted change in the exchange rate for each of the 20 product categories. For most product categories, the exchange rate had decreased from mid 2014 to early 2015. This is reasonable given that most of the imported products are from Russia and its currency had sharply depreciated during the same period as was shown in panel (a) of Figure 1. The exchange rate bounced back during the second half of 2015 following the regime change in the country's exchange rate policy, and became more stable afterwards.

In model (1) we replace the exchange rate, er, with the sales-weighted exchange rate, $e r^{*}$, constructed above, and examine the effect of exchange rate shock on the price of domestic goods. ${ }^{10}$ As expected, the ERPTs for domestic products when $L=5$ presented in Figure 4 are much smaller

\footnotetext{
${ }^{8}$ As an example, suppose there are three imported products in a given product category: two from Russia with combined sales of 100 packages and one from Germany with sales of 50 packages. If the month-to-month exchange rate change is 9 percent between Ruble and Tenge and -6 percent between Euro and Tenge, the sales-weighted change in the exchange rate would be 4 percent $\left(\frac{2}{3} \cdot 9-\frac{1}{3} \cdot 6\right)$ for all the domestic products in the product category.

${ }^{9}$ To address the concern that the exchange rate fluctuations may induce the weight to change over time, we also consider a fixed weight for a given exporting country, that is, the sales share of the country at beginning month of the sample (January 2014). Estimation results are qualitatively unaffected.

${ }^{10} \mathrm{CPI}$ for non-food goods are used in the analysis of the ERPT for domestic products.
} 
Figure 4: ERPT - domestic products

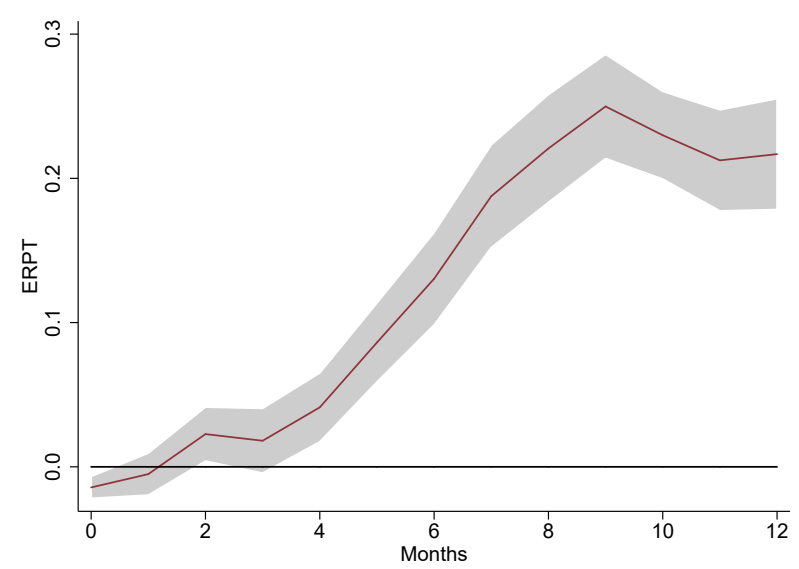

Note: The Figure graphically present the estimates of the ERPTs for domestic products up to $h=12$, that is, up to one year after the shock, for $L=5$ along with 95 percent confidence bands.

in magnitude compared to those of imported products shown in Figure $3 .^{11}$ The pass-through reaches its maximum level of 25 percent after nine months and remains stable thereafter, which is half of the maximum pass-through of the imported product, 50 percent. The result does not change when four and six lags of the control variables are included as can be seen in the bottom panel of Figure A4 in the appendix.

\subsection{Separating the impacts of appreciation and depreciation}

The retail price may react differently to the exchange rate shock, depending on whether the local currency (Tenge) is depreciated or appreciated against the foreign currency. Therefore, now we allow for the asymmetry in the exchange rate pass-through. Specifically, we define the binary variable $d_{i, t}$ that is equal to one if Tenge is depreciated against the currency of the country exporting item $i$ between $t-1$ and $t$, and interact it with $\Delta \ln e r_{i, t}$ :

$$
\begin{aligned}
\Delta_{h} \ln p_{i, t+h} & =\alpha_{i}^{h}+\beta_{1}^{h} \Delta \ln e r_{i, t}+\beta_{2}^{h} \Delta \ln e r_{i, t} * d_{i, t}+\sum_{l=1}^{L} \gamma_{l}^{h} \Delta \ln e r_{i, t-l}+\sum_{l=1}^{L} \delta_{l}^{h} \Delta \ln p_{i, t-l} \\
& +\sum_{l=1}^{L} \lambda_{l}^{h} \mathbf{x}_{i, t-l}+\varepsilon_{i, t+h} .
\end{aligned}
$$

\footnotetext{
${ }^{11}$ Table A3 in the appendix present the full estimation results for $h=0,2, \cdots, 10,12$ and $L=5$.
} 
Figure 5: Asymmetric ERPT

(a) Imported products

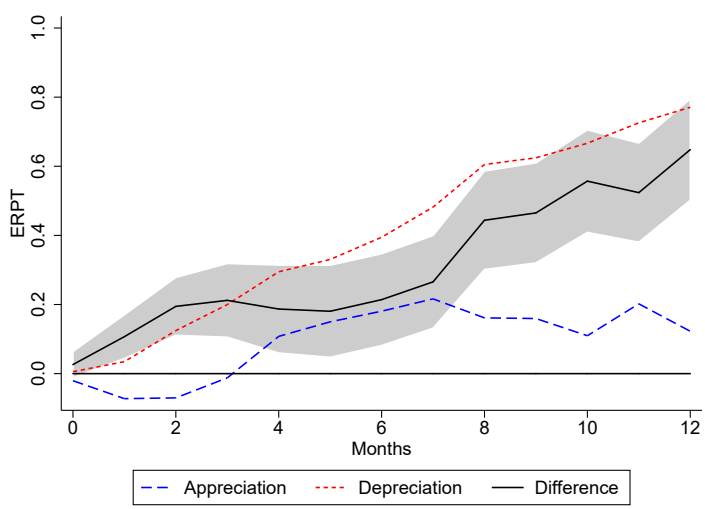

(b) Domestic products

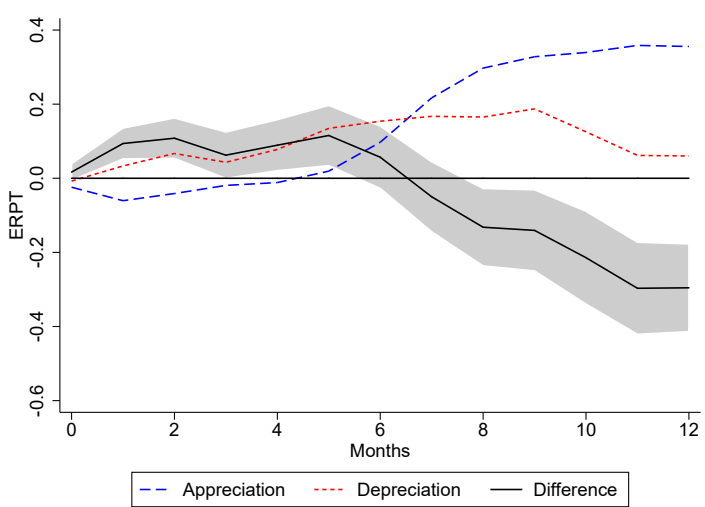

Note: The two panels of the Figure present the estimated cumulative price responses under depreciation and appreciation when $L=5$, and the gap between them along with 95 percent confidence bands.

In this specification, the price responses of imported products to appreciation and depreciation are captured by $\beta_{1}^{h}$ and $\beta_{1}^{h}+\beta_{2}^{h}$, respectively. Therefore, $\kappa_{2}^{h}$ measures the degree of asymmetry in exchange rate pass-through.

The estimated cumulative price response of imported products to the depreciation and appreciation when $L=5$ and the gap between them are presented in the left panel of Figure 5 along with 95 percent confidence bands. ${ }^{12}$ The results yield a sharp contrast between the price responses under depreciation and appreciation of Tenge. The pass-through does not exceed 20 percent after appreciation of Tenge. In case of depreciation of Tenge, however, pass-through consistently increases over time and reaches almost 80 percent one year after the shock. Consequently, there is a persistent gap between the two price responses.

Next, we separate the effect of depreciation on the price of a domestic good from the effect of appreciation using model (2), but replacing er with $e r^{*}$. The estimated cumulative price response of the locally produced goods to the positive and negative exchange rate shocks are presented in the right panel of Figure $5 .{ }^{13}$ The results reveal that price adjustment process is completed within

\footnotetext{
${ }^{12}$ See Table A4 in the appendix for the full estimation results of model (2) for $h=0,2, \cdots, 10,12$ and $L=5$. Also, see the top panel of Figure A5 in the appendix for the price responses of imported products to appreciation and depreciation when $L=4$ and 6 .

${ }^{13}$ The full estimation results for $h=0,2, \cdots, 10,12$ and $L=5$ are presented in Table A5 in the appendix. Also, see the bottom panel of Figure A5 in the appendix for the price responses of domestic products to appreciation and depreciation when $L=4$ and 6 .
} 
Figure 6: Asymmetric Price Reaction Function

(a) Imported products

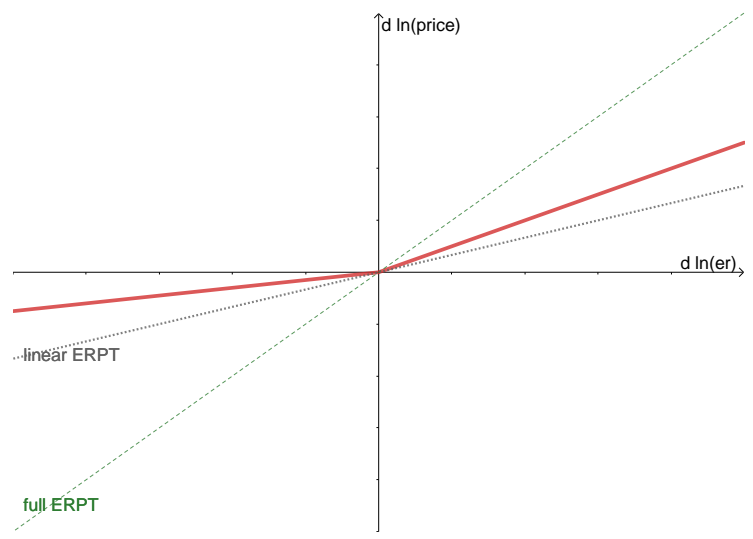

(b) Domestic products

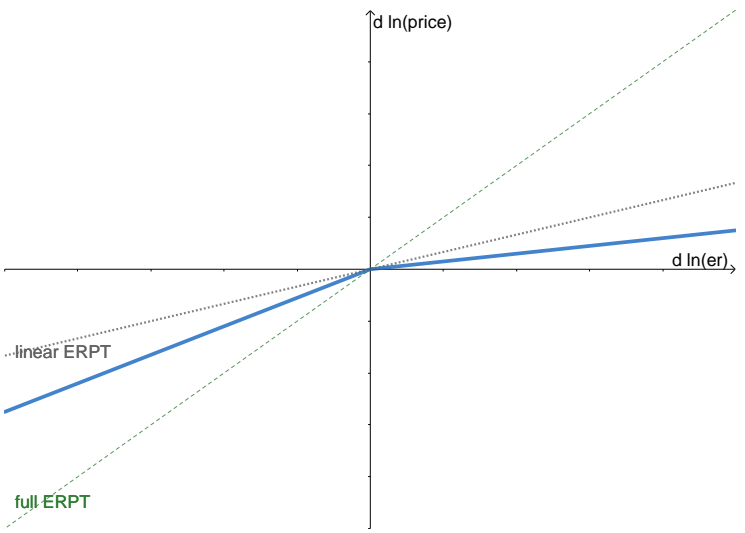

few months after the depreciation of Tenge, while the effect of appreciation is slower but larger; the long-run pass-through is almost 40 percent in case of appreciation of Tenge, while it is always below 20 percent in case of depreciation. Consequently, the gap between the two price responses is positive in the shorter horizons, but become negative on longer horizons.

Hence, we find that the features of exchange rate pass-through for domestic products are the opposite of those for imported products; whereas the pass-through effect is much larger under depreciation than under appreciation for imported products, appreciation passes through much more than depreciation for domestic products. These findings suggest that the piecewise linear price reaction function is convex for imported products, but concave for domestic products, as can be seen in Figure 6. According to Bussierre (2013), convex price reaction function implies producers are more prone to increase their markup, while concavity implies they are more concerned with their competitiveness. Hence, domestic producers may be keen on preserving the market share, while foreign producers may be more concerned with increasing markup. This is consistent with the observation from Table A1 in the appendix that prices are higher for imported products, which suggests that they may have higher quality than domestic goods.

Now, we examine differential effects of the exchange rate shock based on its size. Given that the exchange rate in Kazakhstan shows much wider fluctuations than those observed in developed countries (Figure 2), the usual threshold of 3 percent change for defining large exchange rate shock in the previous literature (Pollard and Coughlin, 2004; Kim, Lewis, and Vigfusson, 2018) may not 
be applicable to our case. Instead, we define more than 10 percent changes in the exchange rate, which is rarely observed in any developed economy, as a large exchange rate shock in the following specification:

$$
\begin{aligned}
\Delta_{h} \ln p_{i, t+h} & =\alpha_{i}^{h}+\beta_{1}^{h} \Delta \ln e r_{i, t}+\beta_{2}^{h} \Delta \ln e r_{i, t} * d_{i, t}+\beta_{3}^{h} \Delta \ln e r_{i, t} * \text { Over } 10_{i}+\beta_{4}^{h} \Delta \ln e r_{i, t} * d_{i, t} * \text { Over } 10_{i} \\
& +\sum_{l=1}^{L} \gamma_{l}^{h} \Delta \ln e r_{i, t-l}+\sum_{l=1}^{L} \delta_{l}^{h} \Delta \ln p_{i, t-l}+\sum_{l=1}^{L} \lambda_{l}^{h} \mathbf{x}_{i, t-l}+\varepsilon_{i, t+h}
\end{aligned}
$$

where a binary variable Over $10_{i}$ that equals one if the absolute value of the exchange rate change exceeds 10 percent. The price responses to small appreciation (less than 10 percent decrease in the exchange rate) and small depreciation (less than 10 percent increase in the exchange rate) are measured by $\beta_{1}^{h}$ and $\beta_{1}^{h}+\beta_{2}^{h}$, respectively, while the price responses to large appreciation and large depreciation in large retailers are captured by $\beta_{1}^{h}+\beta_{3}^{h}$ and $\beta_{1}^{h}+\beta_{2}^{h}+\beta_{3}^{h}+\beta_{4}^{h}$, respectively.

Estimates reported in Figure 7 suggest that while the price response is still asymmetric, it is also non-linear. ${ }^{14}$ According to the left panel of the figure, the price response of an imported good is smaller when the exchange rate shock is large regardless of whether it is appreciation or depreciation. This is the opposite of Pollard and Coughlin (2004), who find that higher exchange rate pass-throughs are associated with larger exchange rate shocks. Given our definition of the 'large' exchange rate shock, that is, the month-to-month exchange rate change that exceeds 10 percent, the results suggest that producers of imported goods are slow to adjust prices when the exchange rate shock is truly large.

The right panel of Figure 7 shows that in case of appreciation, the price response of a domestic product is larger when the size of the exchange rate shock is small. Also, the pass-through of domestic goods tends to be higher than that of imported goods regardless of the size of appreciation, implying that the prices of domestic goods tend to decrease more than imported goods when the Tenge appreciates. In case of depreciation, while the price response of a domestic product is even negative when the size of the exchange rate shock is small, the pass-through is much lower compared to the pass-through for imported goods for both small and large depreciations. These findings are consistent with domestic producers' interest in market share.

\footnotetext{
${ }^{14}$ The full estimation results for $h=0,2, \cdots, 10,12$ and $L=5$ are presented in Tables A6 and A7 in the appendix for imported products and for domestic products, respectively.
} 
Figure 7: Large vs small exchange rate shock

(a) Imported products

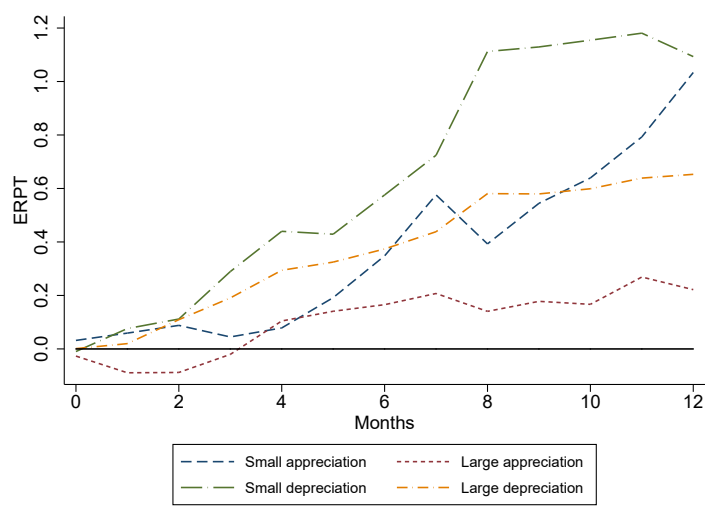

(b) Domestic products

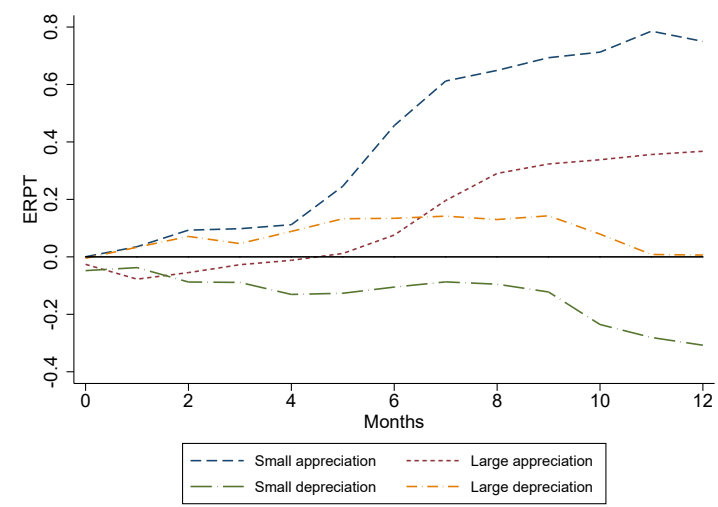

Note: The two panels of the Figure present the estimates of large and small asymmetric ERPTs when $L=5$.

\subsection{Retailer size and ERPT}

The price impact of exchange rate shock may vary depending on the retailer size (Antoniades and Zaniboni, 2016). Here, we examine the relationship between the retailer size and exchange rate pass-through. To do so, we classify the retailers in the data into two groups: (i) large retailers and (ii) small/memdium retailers. ${ }^{15}$ Approximately 20 percent of the observations are from large retailers. Then, we define the binary variable $\operatorname{Large}_{i}$ that is equal to one if item (product-retailer category) $i$ is sold at a large retailer and zero otherwise, and interact it with $e r_{i, t}$ :

$$
\begin{aligned}
\Delta_{h} \ln p_{i, t+h} & =\alpha_{i}^{h}+\kappa_{1}^{h} \Delta \ln e r_{i, t}+\kappa_{2}^{h} \Delta \ln e r_{i, t} * \operatorname{Large}_{i}+\sum_{l=1}^{L} \gamma_{l}^{h} \Delta \ln e r_{i, t-l}+\sum_{l=1}^{L} \delta_{l}^{h} \Delta \ln p_{i, t-l} \\
& +\sum_{l=1}^{L} \lambda_{l}^{h} \mathbf{x}_{i, t-l}+\varepsilon_{i, t+h},
\end{aligned}
$$

where the impact of the exchange rate shock on the price of imported products at small/medium retailers and large retailers are captured by $\kappa_{1}^{h}$ and $\kappa_{1}^{h}+\kappa_{2}^{h}$, respectively.

The left panel of Figure 8 presents the estimates of ERPTs over time when $L=5$ for the two retailer types and the difference between them along with 95 percent confidence bands. ${ }^{16}$ The results show that the impact of the exchange rate shock is different between the two types of

\footnotetext{
15 "Urban Large Food \& Mixed Stores" and "Super/ Large Mixed stores" are classified as large retailers.

${ }^{16}$ Table A8 in the appendix presents the full estimation results of model (4) for $h=0,2, \cdots, 10,12$ and $L=5$. Also, estimates of the ERPTs for imported products when $L=4$ and 6 are presented in the top panel of Figure A6.
} 
Figure 8: ERPT and the retailer size

(a) Imported products

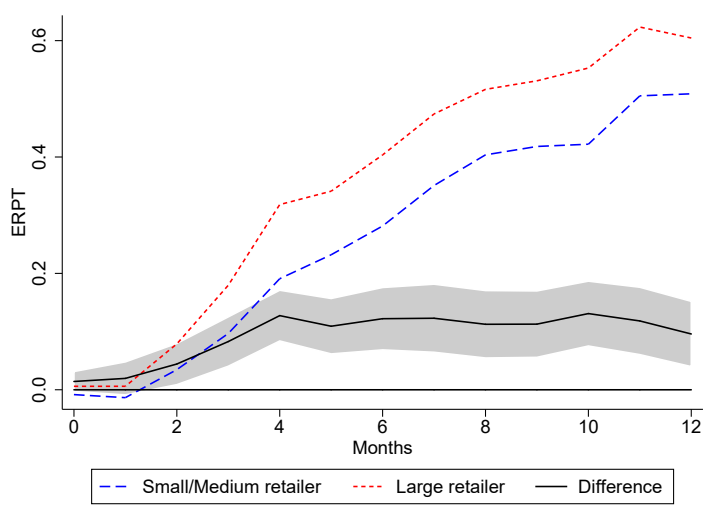

(b) Domestic products

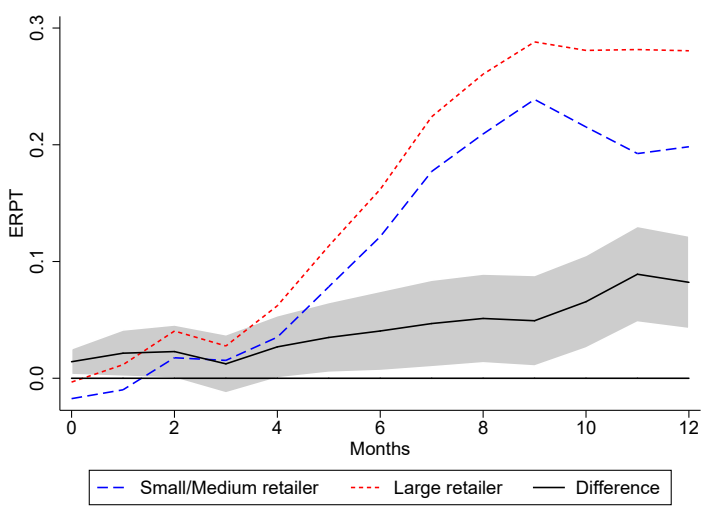

Note: The two panels of the Figure present the estimates of the ERPTs when $L=5$ at large and small/medium retailers, and the gap between them along with 95 percent confidence bands.

retailers. Four months after the shock and thereafter, for example, the pass-through is around 10 percent point higher for a large retailer compared to a small/medium retailer. This is consistent with the findings of the previous works (Corsetti and Dedola, 2005; Hellerstein, 2008; Antoniades and Zaniboni, 2016) and suggest that large retailers may have lower local costs or lower markups, which induces their retail prices to rely more on the exchange rate.

To compare the size of the exchange rate pass-through for domestic products in the two types of retailer, we use the sales-weighted exchange rate, $e r^{*}$, instead of the exchange rate, $e r$, in model (4). Estimates of the ERPTs over time for domestic products sold at the two retailer types are presented in the right panel of Figure 8. Consistent with the case of imported products, the price impact of the exchange rate shock is larger for a large retailer by $5-8$ percent points eight months after the shock and thereafter. ${ }^{17}$

Finally, we differentiate the price impacts of appreciation and depreciation in small/medium

\footnotetext{
${ }^{17}$ The full estimation results for $h=0,2, \cdots, 10,12$ and $L=5$ are presented in Table A9 in the appendix. Also, estimates of the ERPTs for domestic products when $L=4$ and 6 are presented in the bottom panel of Figure A6 in the appendix.
} 
and large retailers:

$$
\begin{aligned}
\Delta_{h} \ln p_{i, t+h} & =\alpha_{i}^{h}+\kappa_{1}^{h} \Delta \ln e r_{i, t}+\kappa_{2}^{h} \Delta \ln e r_{i, t} * d_{i, t}+\kappa_{3}^{h} \Delta \ln e r_{i, t} * \operatorname{Large}_{i}+\kappa_{4}^{h} \Delta \ln \mathrm{er}_{i, t} * d_{i, t} * \text { Large }_{i} \\
& +\sum_{l=1}^{L} \gamma_{l}^{h} \Delta \ln e r_{i, t-l}+\sum_{l=1}^{L} \delta_{l}^{h} \Delta \ln p_{i, t-l}+\sum_{l=1}^{L} \lambda_{l}^{h} \mathbf{x}_{i, t-l}+\varepsilon_{i, t+h}
\end{aligned}
$$

where the price responses to appreciation and depreciation in small/medium retailers are measured by $\kappa_{1}^{h}$ and $\kappa_{1}^{h}+\kappa_{2}^{h}$, respectively, while the price responses to appreciation and depreciation in large retailers are captured by $\kappa_{1}^{h}+\kappa_{3}^{h}$ and $\kappa_{1}^{h}+\kappa_{2}^{h}+\kappa_{3}^{h}+\kappa_{4}^{h}$, respectively.

Estimates of the price responses of imported products and domestic products when $L=5$ are presented in Figure 9. ${ }^{18}$ Two observations are noteworthy. First, for both retailer groups the retail price change is larger (i) under depreciation than under appreciation in case of imported goods (the left panel), (ii) under appreciation than under depreciation in case of domestic goods (the right panel). Therefore, our main findings that (i) the pass-through is asymmetric and (ii) the direction of asymmetry is the opposite between imported and domestic products hold regardless of the retailer size. Second, Figure 9 reveals that the mechanism for the large retailers' high responsiveness to the exchange rate shock shown in Figure 8 is different between imported and domestic products. That is, for imported goods large retailers respond more to the exchange rate shock than small/medium retailers when the exchange rate rises (depreciation), while the degree of pass-through is similar between the two retailer groups when the exchange rate falls (appreciation). For domestic goods, however, large retailers respond more to an appreciations than small/medium retailers, while the price response to a depreciation is similar in magnitude between them.

\section{Robustness}

We check the robustness of our findings in two ways. First, note that by estimating standard errors clustered by product, we assume that the residuals are arbitrarily correlated within a product, but uncorrelated among products. Here, we relax this assumption and estimate standard errors using Driscoll and Kraay (1998)'s nonparametric covariance matrix estimator, which correct for very general forms of cross-sectional and temporal correlation.

\footnotetext{
${ }^{18}$ The full estimation results for $h=0,2, \cdots, 10,12$ and $L=5$ are reported in Tables A10 and A11 in the appendix. Also, Figure A7 presents the estimates of the asymmetric ERPTs when $L=4$ and 6 at large and small/medium retailers.
} 
Figure 9: Asymmetric ERPT and the retailer size

(a) Imported products

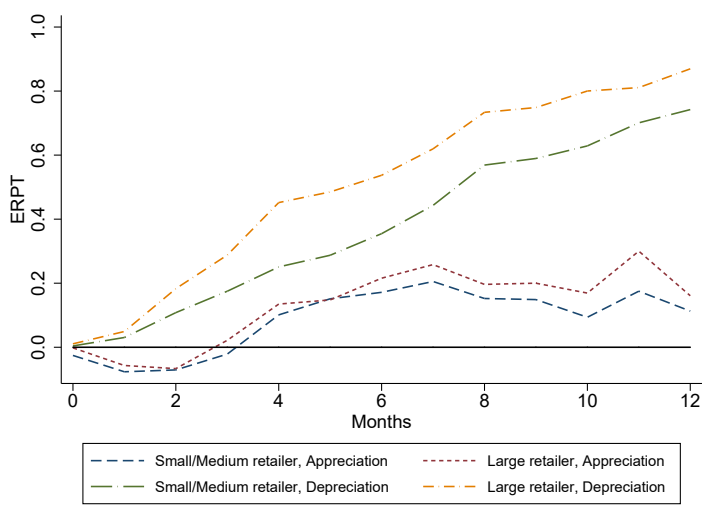

(b) Domestic products

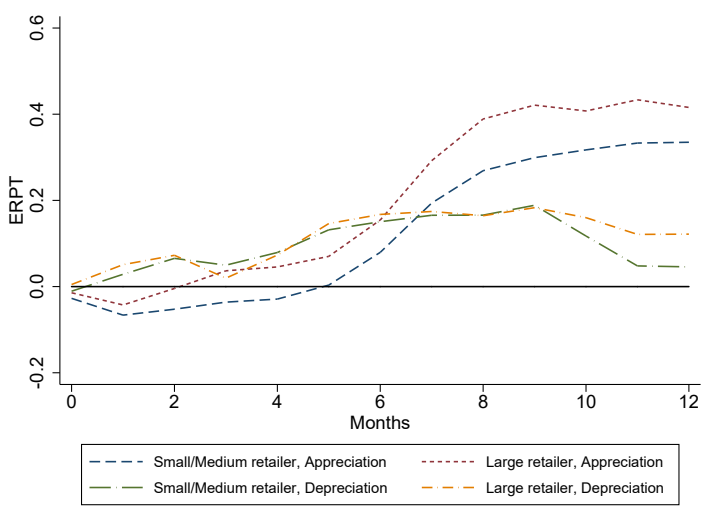

Note: The two panels of the Figure present the estimates of the asymmetric ERPTs at large and small/medium retailers when $L=5$.

Figures A8 and A9 in the appendix graphically present estimated price responses for imported products and for domestic products, respectively. The 95 percent confidence intervals are wider than those in Figures 3 - 5, and hence suggest that Driscoll and Kraay (1998) standard errors are larger than those clustered by product. We argue that Driscoll and Kraay (1998)'s approach may overestimate the standard errors in our case. This is because unobserved common shocks may induce correlation among products in the same category only, while residuals are unlikely to be correlated between product categories. Although the small number of product categories in our data prevents us from clustering by product category, it would yield standard errors larger than those clustered by product but smaller than those under Driscoll and Kraay (1998)'s approach.

Second, we use unbalanced panel of products, adding to our data the products that entered or exited the market during the sample period. Comparing estimated price responses in Figures 3 - 5 with those reported in Figures A10 and A11 in the appendix reveals that using either unbalanced panel and balanced panel leads to estimation results that are qualitatively the same. Therefore, the product replacement bias (Nakamura and Steinsson, 2012) may not be quantitatively important in our case. 


\section{Conclusion}

This papers analyzed ERPT into consumer prices in Kazakhstan between January 2014 and December 2016. This is a particularly interesting period as in August 2015 the exchange rate regime switched from a fixed to a floating system, leading to a large depreciation initially, but to a substantial appreciation later on. To this end, we used a unique scanner data set of more than 1,400 consumer products covering 28 retailer types, including supermarkets and open venues. We found incomplete and heterogeneous pass-through.

In particular, we found higher ERPT for foreign sourced consumer products than for domestic ones. We also found asymmetric pass-through: A depreciation led to higher ERPT than an appreciation for foreign products. But for domestic products a depreciation led to less ERPT than an appreciation. We also provide evidence for non-linear responses. Models taking into account strategic complementarities and heterogeneous responses to shocks in view of competition may provide an intuition for these results. More detailed data, however, on the nature of the rivals and competition at the detailed retailer level are required for this and may provide more detailed insights in the various channels explaining the patterns we documented in this paper.

While the literature has not spent much time documenting asymmetries in ERPT, it is clear that this has potentially important policy implications, especially for high inflation countries, such

as Kazakhstan. In particular, for inflation targeting, these non-linear and asymmetric kind of responses to exchange rate shocks matter. Also, the nature of product composition plays a role, which can have an impact on the welfare impact of shocks for consumers. 


\section{References}

Amiti, M., O. Itskhoki, and J. Konings (2014): "Importers, exporters, and exchange rate disconnect," The American Economic Review, 104(7), 1942-1978.

Antoniades, A., And N. Zaniboni (2016): "Exchange rate pass-through into retail prices," International Economic Review, 57(4), 1425-1447.

AtKeson, A., And A. Burstein (2008): "Pricing-to-market, trade costs, and international relative prices," American Economic Review, 98(5), 1998-2031.

Bussiere, M. (2013): "Exchange rate pass-through to trade prices: The role of nonlinearities and asymmetries," Oxford Bulletin of Economics and Statistics, 75(5), 731-758.

Colicev, A., J. Hoste, And J. Konings (2019): "Exchange Rate Pass-through after a Large Depreciation," Discussion paper.

Corsetti, G., and L. Dedola (2005): "A macroeconomic model of international price discrimination," Journal of International Economics, 67(1), 129-155.

Driscoll, J. C., AND A. C. KraAy (1998): "Consistent covariance matrix estimation with spatially dependent panel data," Review of economics and statistics, 80(4), 549-560.

Goldberg, L. S., And J. M. CAmpa (2010): "The sensitivity of the CPI to exchange rates: Distribution margins, imported inputs, and trade exposure," The Review of Economics and Statistics, 92(2), 392-407.

Gopinath, G., and R. Rigobon (2008): "Sticky borders," The Quarterly Journal of Economics, $123(2), 531-575$.

Hellerstein, R. (2008): "Who bears the cost of a change in the exchange rate? Pass-through accounting for the case of beer," Journal of international economics, 76(1), 14-32.

JoRDÀ, Ò. (2005): "Estimation and inference of impulse responses by local projections," American economic review, 95(1), 161-182.

Kim, M., L. Lewis, And R. Vigfusson (2018): "Asymmetries and non-linearities in exchange rate pass-through," in Inflation: Drivers and Dynamics 2019 conference at the Federal Reserve Bank of Cleveland.

Klenow, P. J., And O. Kryvtsov (2008): "State-dependent or time-dependent pricing: Does it matter for recent US inflation?," The Quarterly Journal of Economics, 123(3), 863-904.

NAKAmura, E., And J. Steinsson (2012): "Lost in transit: product replacement bias and pricing to market," American Economic Review, 102(7), 3277-3316.

Peltzman, S. (2000): "Prices rise faster than they fall," Journal of political economy, 108(3), $466-502$.

Pollard, P., and C. Coughlin (2004): "Size matters: asymmetric exchange rate pass-through at the industry level," Discussion paper, Federal Reserve Bank of St. Louis. 


\section{Appendix}

\subsection{Additional Tables and Figures}

Table A1: Price comparison between domestic and imported products

\begin{tabular}{lrrr}
\hline \hline & \multicolumn{2}{c}{ Average unit price } & Difference $(\%)$ \\
\cline { 2 - 3 } Product category & 73.95 & & \\
\cline { 2 - 3 } Baby diapers & 337.62 & 439.92 & \\
Beer & $1,406.40$ & $1,630.83$ & 26.31 \\
Cheese curds & 12.79 & 13.66 & 14.78 \\
Cigarette & $1,161.09$ & $1,325.85$ & 6.56 \\
Cultured milk & 278.96 & 401.88 & 13.25 \\
Dessert \& pudding & 506.53 & 630.70 & 36.11 \\
Draught beer & 506.53 & 630.70 & 21.84 \\
Drink yogurt refrigerate & 481.65 & 870.95 & 21.84 \\
Energy drink & 491.09 & 686.16 & 57.56 \\
Evaporated milk & 194.89 & 330.83 & 33.14 \\
Fresh refrigerated & 234.73 & 259.78 & 51.71 \\
Fruit \& vegetable juice & $1,701.93$ & $2,261.45$ & 10.13 \\
Glazed curds & 219.49 & & 28.23 \\
Ice tea & 740.05 & 388.00 & -62.42 \\
Juice with milk additive & 246.19 & 301.41 & 20.17 \\
Liquid milk products & 112.38 & 494.21 & 125.90 \\
Mineral water & 877.40 & $1,534.98$ & 54.52 \\
Noodles dry with extra flavouring & 415.16 & 409.48 & -1.38 \\
Pasta dry & 178.83 & 190.77 & 6.46 \\
Soft drinks & 878.64 & 977.37 & 10.64 \\
Sour cream & & $4,503.50$ & \\
Toothpastes & 751.03 & 683.73 & \\
Yogurt spoonable & 0 & \\
\hline \hline
\end{tabular}

Note: Unit price is the price per kilo or liter. Difference (\%) is calculated as $\frac{\text { Imported unit price-Domestic unit price }}{\text { (Imported unit price+Domestic unit price)/2 }} * 100$. 
Table A2: ERPTs for imported products with $L=5$

\begin{tabular}{|c|c|c|c|c|c|c|c|}
\hline \multirow[b]{2}{*}{ Variable } & \multicolumn{7}{|c|}{ Horizon (h) } \\
\hline & 0 & 2 & 4 & 6 & 8 & 10 & 12 \\
\hline$\Delta \ln e r$ & $\begin{array}{l}-0.005 \\
(0.006)\end{array}$ & $\begin{array}{c}0.044^{* * *} \\
(0.012)\end{array}$ & $\begin{array}{c}0.219 * * * \\
(0.019)\end{array}$ & $\begin{array}{c}0.308^{* * *} * \\
(0.021)\end{array}$ & $\begin{array}{c}0.429 * * * \\
(0.023)\end{array}$ & $\begin{array}{c}0.451^{* * *} \\
(0.022)\end{array}$ & $\begin{array}{c}0.530 * * * \\
(0.031)\end{array}$ \\
\hline $\operatorname{lag} 1$ & $\begin{array}{c}0.034^{* * * *} \\
(0.009)\end{array}$ & $\begin{array}{c}0.149^{* * *} \\
(0.011)\end{array}$ & $\begin{array}{c}0.200^{* * *} * \\
(0.013)\end{array}$ & $\begin{array}{c}0.278^{* * *} \\
(0.014)\end{array}$ & $\begin{array}{c}0.275^{* * *} \\
(0.015)\end{array}$ & $\begin{array}{c}0.288^{* * *} \\
(0.018)\end{array}$ & $\begin{array}{c}0.165^{* * *} \\
(0.012)\end{array}$ \\
\hline $\operatorname{lag} 2$ & $\begin{array}{c}0.104^{* * *} \\
(0.010)\end{array}$ & $\begin{array}{c}0.223^{* * *} \\
(0.012)\end{array}$ & $\begin{array}{c}0.245^{* * *} \\
(0.011)\end{array}$ & $\begin{array}{c}0.289^{* * *} \\
(0.013)\end{array}$ & $\begin{array}{c}0.292^{* * *} \\
(0.014)\end{array}$ & $\begin{array}{c}0.236^{* * *} \\
(0.016)\end{array}$ & $\begin{array}{c}0.050^{* *} \\
(0.020)\end{array}$ \\
\hline $\operatorname{lag} 3$ & $\begin{array}{c}0.052^{* * *} \\
(0.008)\end{array}$ & $\begin{array}{c}0.109^{* * *} \\
(0.010)\end{array}$ & $\begin{array}{c}0.202^{* * *} \\
(0.013)\end{array}$ & $\begin{array}{c}0.235^{* * *} * \\
(0.012)\end{array}$ & $\begin{array}{c}0.297^{* * *} \\
(0.016)\end{array}$ & $\begin{array}{c}0.285^{* * *} \\
(0.014)\end{array}$ & $\begin{array}{c}0.202^{* * *} \\
(0.014)\end{array}$ \\
\hline $\operatorname{lag} 4$ & $\begin{array}{c}0.054^{* * *} \\
(0.008)\end{array}$ & $\begin{array}{c}0.094^{* * *} \\
(0.010)\end{array}$ & $\begin{array}{c}0.139 * * * \\
(0.009)\end{array}$ & $\begin{array}{c}0.218^{* * *} \\
(0.011)\end{array}$ & $\begin{array}{c}0.212^{* * *} \\
(0.013)\end{array}$ & $\begin{array}{c}0.219^{* * *} \\
(0.014)\end{array}$ & $\begin{array}{c}0.199 * * * \\
(0.032)\end{array}$ \\
\hline $\operatorname{lag} 5$ & $\begin{array}{c}0.057^{* * *} * \\
(0.008)\end{array}$ & $\begin{array}{c}0.152^{* * *} \\
(0.013)\end{array}$ & $\begin{array}{c}0.189^{* * *} * \\
(0.013)\end{array}$ & $\begin{array}{c}0.222^{* * *} * \\
(0.015)\end{array}$ & $\begin{array}{c}0.201^{* * *} \\
(0.016)\end{array}$ & $\begin{array}{c}0.059^{* * *} \\
(0.013)\end{array}$ & $\begin{array}{c}-0.462^{* * *} \\
(0.056)\end{array}$ \\
\hline \multicolumn{8}{|l|}{$\Delta \ln p$} \\
\hline $\operatorname{lag} 1$ & $\begin{array}{c}-0.214^{* * *} \\
(0.017)\end{array}$ & $\begin{array}{c}-0.349 * * * \\
(0.018)\end{array}$ & $\begin{array}{c}-0.422^{* * *} \\
(0.020)\end{array}$ & $\begin{array}{c}-0.594^{* * *} \\
(0.021)\end{array}$ & $\begin{array}{c}-0.716^{* * *} \\
(0.020)\end{array}$ & $\begin{array}{c}-0.800 * * * \\
(0.023)\end{array}$ & $\begin{array}{c}-0.822^{* * *} \\
(0.024)\end{array}$ \\
\hline $\operatorname{lag} 2$ & $\begin{array}{c}-0.129^{* * *} \\
(0.014)\end{array}$ & $\begin{array}{c}-0.214^{* * *} \\
(0.016)\end{array}$ & $\begin{array}{c}-0.343^{* * *} \\
(0.017)\end{array}$ & $\begin{array}{c}-0.543^{* * *} \\
(0.019)\end{array}$ & $\begin{array}{c}-0.654^{* * *} \\
(0.019)\end{array}$ & $\begin{array}{c}-0.748^{* * *} \\
(0.025)\end{array}$ & $\begin{array}{c}-0.766^{* * *} \\
(0.026)\end{array}$ \\
\hline $\operatorname{lag} 3$ & $\begin{array}{c}-0.060^{* * *} \\
(0.011)\end{array}$ & $\begin{array}{c}-0.138^{* * *} \\
(0.016)\end{array}$ & $\begin{array}{c}-0.309^{* * *} \\
(0.018)\end{array}$ & $\begin{array}{c}-0.479 * * * \\
(0.018)\end{array}$ & $\begin{array}{c}-0.609^{* * *} \\
(0.020)\end{array}$ & $\begin{array}{c}-0.705^{* * *} \\
(0.023)\end{array}$ & $\begin{array}{c}-0.758^{* * *} \\
(0.031)\end{array}$ \\
\hline $\operatorname{lag} 4$ & $\begin{array}{c}-0.027^{* *} \\
(0.011)\end{array}$ & $\begin{array}{c}-0.121^{* * *} \\
(0.015)\end{array}$ & $\begin{array}{c}-0.286^{* * * *} \\
(0.018)\end{array}$ & $\begin{array}{c}-0.408^{* * * *} \\
(0.018)\end{array}$ & $\begin{array}{c}-0.545^{* * *} \\
(0.019)\end{array}$ & $\begin{array}{c}-0.665^{* * *} \\
(0.023)\end{array}$ & $\begin{array}{c}-0.769 * * * \\
(0.034)\end{array}$ \\
\hline $\operatorname{lag} 5$ & $\begin{array}{l}-0.013 \\
(0.010)\end{array}$ & $\begin{array}{c}-0.123^{* * *} \\
(0.014)\end{array}$ & $\begin{array}{c}-0.243^{* * *} \\
(0.016)\end{array}$ & $\begin{array}{c}-0.358^{* * *} \\
(0.017)\end{array}$ & $\begin{array}{c}-0.463^{* * *} \\
(0.018)\end{array}$ & $\begin{array}{c}-0.558^{* * *} \\
(0.024)\end{array}$ & $\begin{array}{c}-0.656^{* * *} \\
(0.037)\end{array}$ \\
\hline \multicolumn{8}{|l|}{$\Delta \ln q$} \\
\hline $\operatorname{lag} 1$ & & $\begin{array}{l}-0.002 \\
(0.002)\end{array}$ & $\begin{array}{c}-0.004^{* *} \\
(0.002)\end{array}$ & $\begin{array}{c}-0.004^{*} \\
(0.002)\end{array}$ & $\begin{array}{l}-0.003 \\
(0.002)\end{array}$ & $\begin{array}{l}-0.000 \\
(0.002)\end{array}$ & $\begin{array}{c}0.003 \\
(0.002)\end{array}$ \\
\hline $\operatorname{lag} 2$ & $\begin{array}{c}-0.002^{*} \\
(0.001)\end{array}$ & $\begin{array}{l}-0.002 \\
(0.002)\end{array}$ & $\begin{array}{l}-0.003 \\
(0.002)\end{array}$ & $\begin{array}{l}-0.002 \\
(0.002)\end{array}$ & $\begin{array}{l}-0.003 \\
(0.002)\end{array}$ & $\begin{array}{c}0.001 \\
(0.002)\end{array}$ & $\begin{array}{c}0.004 \\
(0.002)\end{array}$ \\
\hline $\operatorname{lag} 3$ & $\begin{array}{l}-0.000 \\
(0.001)\end{array}$ & $\begin{array}{l}-0.002 \\
(0.002)\end{array}$ & $\begin{array}{l}-0.001 \\
(0.002)\end{array}$ & $\begin{array}{l}-0.001 \\
(0.002)\end{array}$ & $\begin{array}{c}0.001 \\
(0.002)\end{array}$ & $\begin{array}{c}0.004 \\
(0.002)\end{array}$ & $\begin{array}{c}0.003 \\
(0.002)\end{array}$ \\
\hline $\operatorname{lag} 4$ & $\begin{array}{l}-0.000 \\
(0.001)\end{array}$ & $\begin{array}{l}-0.001 \\
(0.002)\end{array}$ & $\begin{array}{c}-0.000 \\
(0.002)\end{array}$ & $\begin{array}{c}-0.002 \\
(0.002)\end{array}$ & $\begin{array}{c}0.002 \\
(0.002)\end{array}$ & $\begin{array}{c}0.002 \\
(0.003)\end{array}$ & $\begin{array}{c}0.008^{* * *} \\
(0.002)\end{array}$ \\
\hline $\operatorname{lag} 5$ & $\begin{array}{l}-0.001 \\
(0.001)\end{array}$ & $\begin{array}{l}-0.001 \\
(0.001)\end{array}$ & $\begin{array}{l}-0.002 \\
(0.002)\end{array}$ & $\begin{array}{c}0.000 \\
(0.002)\end{array}$ & $\begin{array}{c}0.001 \\
(0.002)\end{array}$ & $\begin{array}{l}-0.000 \\
(0.002)\end{array}$ & $\begin{array}{c}0.003 \\
(0.002)\end{array}$ \\
\hline \multicolumn{8}{|l|}{$\Delta \ln C P I$} \\
\hline $\operatorname{lag} 1$ & $\begin{array}{c}0.807^{* * *} \\
(0.091)\end{array}$ & $\begin{array}{c}1.013^{* * *} \\
(0.156)\end{array}$ & $\begin{array}{c}1.444^{* * *} \\
(0.168)\end{array}$ & $\begin{array}{c}1.872^{* * *} \\
(0.201)\end{array}$ & $\begin{array}{c}2.779^{* * *} \\
(0.255)\end{array}$ & $\begin{array}{c}3.645^{* * *} \\
(0.380)\end{array}$ & $\begin{array}{c}2.292^{* * * *} \\
(0.218)\end{array}$ \\
\hline $\operatorname{lag} 2$ & $\begin{array}{l}-0.121 \\
(0.092)\end{array}$ & $\begin{array}{l}-0.139 \\
(0.121)\end{array}$ & $\begin{array}{c}-0.504^{* * *} \\
(0.120)\end{array}$ & $\begin{array}{c}-0.327^{* * * *} \\
(0.106)\end{array}$ & $\begin{array}{l}-0.065 \\
(0.168)\end{array}$ & $\begin{array}{l}-0.162 \\
(0.141)\end{array}$ & $\begin{array}{c}-0.991 * * * \\
(0.279)\end{array}$ \\
\hline $\operatorname{lag} 3$ & $\begin{array}{l}-0.029 \\
(0.103)\end{array}$ & $\begin{array}{l}-0.022 \\
(0.119)\end{array}$ & $\begin{array}{c}-0.405^{* * *} \\
(0.142)\end{array}$ & $\begin{array}{c}-0.369 * * * \\
(0.139)\end{array}$ & $\begin{array}{l}-0.156 \\
(0.107)\end{array}$ & $\begin{array}{c}0.118 \\
(0.118)\end{array}$ & $\begin{array}{c}-0.664^{* * *} \\
(0.131)\end{array}$ \\
\hline $\operatorname{lag} 4$ & $\begin{array}{c}0.109 \\
(0.091)\end{array}$ & $\begin{array}{c}-0.539 * * * \\
(0.123)\end{array}$ & $\begin{array}{c}-0.741^{* * *} \\
(0.145)\end{array}$ & $\begin{array}{c}-0.795^{* * *} \\
(0.167)\end{array}$ & $\begin{array}{c}-0.955^{* * * *} \\
(0.168)\end{array}$ & $\begin{array}{c}-1.235^{* * * *} \\
(0.171)\end{array}$ & $\begin{array}{c}-2.759 * * * \\
(0.363)\end{array}$ \\
\hline $\operatorname{lag} 5$ & $\begin{array}{c}-0.802^{* * *} \\
(0.085)\end{array}$ & $\begin{array}{c}-1.343^{* * *} \\
(0.131)\end{array}$ & $\begin{array}{c}-1.018^{* * *} \\
(0.164)\end{array}$ & $\begin{array}{l}-0.241 \\
(0.213)\end{array}$ & $\begin{array}{c}0.962^{* * *} \\
(0.222)\end{array}$ & $\begin{array}{c}1.226^{* * *} \\
(0.241)\end{array}$ & $\begin{array}{c}0.597^{* * *} \\
(0.191)\end{array}$ \\
\hline $\begin{array}{l}\text { Fixed effects } \\
\text { Item }\end{array}$ & Yes & Yes & Yes & Yes & Yes & Yes & Yes \\
\hline$R$-squared & 0.092 & 0.216 & 0.336 & 0.434 & 0.490 & 0.509 & 0.528 \\
\hline Observations & 25,740 & 24,024 & 22,308 & 20,592 & 18,876 & 17,160 & 15,444 \\
\hline
\end{tabular}

Note: The table presents the full estimation results of model (1) for $h=0,2, \cdots, 10,12$ and $L=5$. In case of $h=0$, the dependent variable, $\ln p_{t}-\ln p_{t-1}$, would be correlated with the lagged variable $\ln q_{t-1}-\ln q_{t-2}$. Therefore, for $h=0$ the model includes $\Delta \ln q_{i, t-l}$ for $l$ from 2 to $L$. Robust standard errors (clustered by product) are in parentheses. The notation $* * *$ indicates significance at $1 \%$ level, ${ }^{* *}$ at $5 \%$ level, ${ }^{*}$ at $10 \%$ level. 
Table A3: ERPTs for domestic products $L=5$

\begin{tabular}{|c|c|c|c|c|c|c|c|}
\hline \multirow[b]{2}{*}{ Variable } & \multicolumn{7}{|c|}{ Horizon (h) } \\
\hline & 0 & 2 & 4 & 6 & 8 & 10 & 12 \\
\hline$\Delta \ln e r^{*}$ & $\begin{array}{c}-0.014^{* * *} \\
(0.004)\end{array}$ & $\begin{array}{c}0.023^{* *} \\
(0.010)\end{array}$ & $\begin{array}{c}0.041^{* * *} \\
(0.012)\end{array}$ & $\begin{array}{c}0.130^{* * *} \\
(0.016)\end{array}$ & $\begin{array}{c}0.221^{* * *} \\
(0.019)\end{array}$ & $\begin{array}{c}0.230^{* * *} \\
(0.016)\end{array}$ & $\begin{array}{c}0.217^{* * *} \\
(0.020)\end{array}$ \\
\hline $\operatorname{lag} 1$ & $\begin{array}{c}0.024^{* * *} \\
(0.006)\end{array}$ & $\begin{array}{c}0.033^{* * *} \\
(0.006)\end{array}$ & $\begin{array}{c}0.099 * * * \\
(0.007)\end{array}$ & $\begin{array}{c}0.135^{* * *} \\
(0.008)\end{array}$ & $\begin{array}{c}0.119 * * * \\
(0.007)\end{array}$ & $\begin{array}{c}0.087 * * * \\
(0.009)\end{array}$ & $\begin{array}{c}0.028^{* * *} \\
(0.007)\end{array}$ \\
\hline $\operatorname{lag} 2$ & $\begin{array}{c}-0.030 * * * \\
(0.006)\end{array}$ & $\begin{array}{c}-0.025^{* * *} \\
(0.009)\end{array}$ & $\begin{array}{c}-0.041^{* * *} \\
(0.010)\end{array}$ & $\begin{array}{c}0.011 \\
(0.011)\end{array}$ & $\begin{array}{c}-0.042^{* *} \\
(0.016)\end{array}$ & $\begin{array}{c}-0.024^{* *} \\
(0.012)\end{array}$ & $\begin{array}{c}-0.025^{*} \\
(0.015)\end{array}$ \\
\hline $\operatorname{lag} 3$ & $\begin{array}{c}0.002 \\
(0.006)\end{array}$ & $\begin{array}{c}-0.016^{*} \\
(0.009)\end{array}$ & $\begin{array}{c}0.044^{* * *} \\
(0.012)\end{array}$ & $\begin{array}{c}0.100 * * * \\
(0.014)\end{array}$ & $\begin{array}{c}0.193 * * * \\
(0.020)\end{array}$ & $\begin{array}{c}0.219 * * * \\
(0.017)\end{array}$ & $\begin{array}{c}0.031^{* *} \\
(0.014)\end{array}$ \\
\hline $\operatorname{lag} 4$ & $\begin{array}{c}-0.015^{* *} \\
(0.007)\end{array}$ & $\begin{array}{c}0.024^{* * *} \\
(0.008)\end{array}$ & $\begin{array}{c}0.109 * * * \\
(0.009)\end{array}$ & $\begin{array}{c}0.156^{* * *} \\
(0.012)\end{array}$ & $\begin{array}{c}0.212^{* * *} \\
(0.015)\end{array}$ & $\begin{array}{c}0.075^{* * *} \\
(0.010)\end{array}$ & $\begin{array}{c}0.097^{* * *} \\
(0.015)\end{array}$ \\
\hline $\operatorname{lag} 5$ & $\begin{array}{c}0.025 * * * \\
(0.005)\end{array}$ & $\begin{array}{c}0.109^{* * *} \\
(0.010)\end{array}$ & $\begin{array}{c}0.136^{* * *} \\
(0.013)\end{array}$ & $\begin{array}{c}0.136^{* * *} \\
(0.018)\end{array}$ & $\begin{array}{c}-0.043^{* *} \\
(0.021)\end{array}$ & $\begin{array}{c}-0.145^{* * *} \\
(0.015)\end{array}$ & $\begin{array}{c}-0.217 * * * \\
(0.026)\end{array}$ \\
\hline $\begin{array}{l}\Delta \ln p \\
\quad \operatorname{lag} 1\end{array}$ & $-0.132^{* * *}$ & $-0.294^{* * *}$ & $-0.362^{* * *}$ & $-0.479^{* * *}$ & $-0.580^{* * *}$ & $-0.689^{* * *}$ & $-0.729 * * *$ \\
\hline $\operatorname{lag} 2$ & $\begin{array}{c}(0.016) \\
-0.126^{* * *} \\
(0.012)\end{array}$ & $\begin{array}{c}(0.021) \\
-0.216^{* * *} \\
(0.017)\end{array}$ & $\begin{array}{c}(0.022) \\
-0.335^{* * *} \\
(0.020)\end{array}$ & $\begin{array}{c}(0.021) \\
-0.439 * * * \\
(0.020)\end{array}$ & $\begin{array}{c}(0.021) \\
-0.557^{* * *} \\
(0.023)\end{array}$ & $\begin{array}{c}(0.023) \\
-0.614^{* * *} \\
(0.028)\end{array}$ & $\begin{array}{c}(0.025) \\
-0.701 * * * \\
(0.029)\end{array}$ \\
\hline $\operatorname{lag} 3$ & $\begin{array}{c}-0.075^{* * *} \\
(0.008)\end{array}$ & $\begin{array}{c}-0.162^{* * *} \\
(0.015)\end{array}$ & $\begin{array}{c}-0.276^{* * *} \\
(0.017)\end{array}$ & $\begin{array}{c}-0.393^{* * *} * \\
(0.019)\end{array}$ & $\begin{array}{c}-0.487^{* * *} \\
(0.023)\end{array}$ & $\begin{array}{c}-0.542^{* * *} \\
(0.027)\end{array}$ & $\begin{array}{c}-0.600^{* * * *} \\
(0.034)\end{array}$ \\
\hline $\operatorname{lag} 4$ & $\begin{array}{c}-0.035^{* * *} \\
(0.009)\end{array}$ & $\begin{array}{c}-0.137^{* * * *} \\
(0.015)\end{array}$ & $\begin{array}{c}-0.222^{* * * *} \\
(0.016)\end{array}$ & $\begin{array}{c}-0.327^{* * * *} \\
(0.019)\end{array}$ & $\begin{array}{c}-0.403^{* * *} \\
(0.025)\end{array}$ & $\begin{array}{c}-0.502^{* * * *} \\
(0.029)\end{array}$ & $\begin{array}{c}-0.560^{* * * *} \\
(0.034)\end{array}$ \\
\hline $\operatorname{lag} 5$ & $\begin{array}{c}-0.046^{* * *} \\
(0.009)\end{array}$ & $\begin{array}{c}-0.128^{* * *} \\
(0.013)\end{array}$ & $\begin{array}{c}-0.214^{* * *} \\
(0.016)\end{array}$ & $\begin{array}{c}-0.291^{* * *} \\
(0.020)\end{array}$ & $\begin{array}{c}-0.358^{* * *} \\
(0.025)\end{array}$ & $\begin{array}{c}-0.406^{* * *} \\
(0.030)\end{array}$ & $\begin{array}{c}-0.449^{* * *} \\
(0.032)\end{array}$ \\
\hline$\Delta \ln q$ & & & & & & & \\
\hline $\operatorname{lag} 1$ & & $\begin{array}{c}0.004^{* * *} \\
(0.001)\end{array}$ & $\begin{array}{c}0.003^{* *} \\
(0.001)\end{array}$ & $\begin{array}{c}0.002 \\
(0.002)\end{array}$ & $\begin{array}{c}0.002 \\
(0.002)\end{array}$ & $\begin{array}{c}0.006^{* * *} \\
(0.002)\end{array}$ & $\begin{array}{c}0.008^{* * *} \\
(0.003)\end{array}$ \\
\hline $\operatorname{lag} 2$ & $\begin{array}{c}0.003^{* * *} \\
(0.001)\end{array}$ & $\begin{array}{c}0.003^{* *} \\
(0.001)\end{array}$ & $\begin{array}{c}0.002 \\
(0.001)\end{array}$ & $\begin{array}{l}-0.000 \\
(0.002)\end{array}$ & $\begin{array}{c}0.002 \\
(0.002)\end{array}$ & $\begin{array}{c}0.006^{* *} \\
(0.003)\end{array}$ & $\begin{array}{c}0.009 * * * \\
(0.003)\end{array}$ \\
\hline $\operatorname{lag} 3$ & $\begin{array}{c}0.001 \\
(0.001)\end{array}$ & $\begin{array}{c}0.001 \\
(0.001)\end{array}$ & $\begin{array}{l}-0.000 \\
(0.002)\end{array}$ & $\begin{array}{l}-0.002 \\
(0.002)\end{array}$ & $\begin{array}{c}0.001 \\
(0.002)\end{array}$ & $\begin{array}{c}0.004 \\
(0.003)\end{array}$ & $\begin{array}{c}0.008^{* *} \\
(0.003)\end{array}$ \\
\hline $\operatorname{lag} 4$ & $\begin{array}{c}0.000 \\
(0.001)\end{array}$ & $\begin{array}{c}0.001 \\
(0.001)\end{array}$ & $\begin{array}{l}-0.002 \\
(0.002)\end{array}$ & $\begin{array}{l}-0.000 \\
(0.002)\end{array}$ & $\begin{array}{c}0.000 \\
(0.003)\end{array}$ & $\begin{array}{c}0.004 \\
(0.003)\end{array}$ & $\begin{array}{l}0.006^{*} \\
(0.003)\end{array}$ \\
\hline $\operatorname{lag} 5$ & $\begin{array}{c}0.000 \\
(0.001)\end{array}$ & $\begin{array}{l}-0.001 \\
(0.001)\end{array}$ & $\begin{array}{c}-0.003^{*} \\
(0.001)\end{array}$ & $\begin{array}{c}0.001 \\
(0.002)\end{array}$ & $\begin{array}{c}0.001 \\
(0.002)\end{array}$ & $\begin{array}{l}0.004^{*} \\
(0.003)\end{array}$ & $\begin{array}{c}0.002 \\
(0.003)\end{array}$ \\
\hline$\Delta \ln C P I$ & & & & & & & \\
\hline $\operatorname{lag} 1$ & $\begin{array}{c}0.652^{* * *} \\
(0.044)\end{array}$ & $\begin{array}{c}0.873^{* * *} \\
(0.062)\end{array}$ & $\begin{array}{c}1.446^{* * *} \\
(0.086)\end{array}$ & $\begin{array}{c}0.890^{* * *} \\
(0.096)\end{array}$ & $\begin{array}{c}0.725^{* * *} \\
(0.126)\end{array}$ & $\begin{array}{c}0.177^{* *} \\
(0.088)\end{array}$ & $\begin{array}{c}0.043 \\
(0.098)\end{array}$ \\
\hline $\operatorname{lag} 2$ & $\begin{array}{c}-0.320^{* * *} \\
(0.053)\end{array}$ & $\begin{array}{c}0.421^{* * *} \\
(0.060)\end{array}$ & $\begin{array}{c}0.063 \\
(0.071)\end{array}$ & $\begin{array}{c}-0.380^{* * * *} \\
(0.078)\end{array}$ & $\begin{array}{c}-0.733^{* * *} \\
(0.092)\end{array}$ & $\begin{array}{c}-0.873^{* * * *} \\
(0.083)\end{array}$ & $\begin{array}{c}0.087 \\
(0.072)\end{array}$ \\
\hline $\operatorname{lag} 3$ & $\begin{array}{c}0.631^{* * *} \\
(0.060)\end{array}$ & $\begin{array}{c}0.575^{* * *} * \\
(0.057)\end{array}$ & $\begin{array}{c}0.218^{* * *} \\
(0.077)\end{array}$ & $\begin{array}{c}-0.187^{* *} \\
(0.082)\end{array}$ & $\begin{array}{c}-0.614^{* * *} \\
(0.097)\end{array}$ & $\begin{array}{c}0.069 \\
(0.076)\end{array}$ & $\begin{array}{c}1.861^{* * *} \\
(0.280)\end{array}$ \\
\hline $\operatorname{lag} 4$ & $\begin{array}{l}-0.074 \\
(0.049)\end{array}$ & $\begin{array}{c}-0.143^{* *} \\
(0.062)\end{array}$ & $\begin{array}{c}-0.652^{* * *} \\
(0.076)\end{array}$ & $\begin{array}{c}-0.490^{* * *} \\
(0.092)\end{array}$ & $\begin{array}{c}0.406^{* * *} \\
(0.104)\end{array}$ & $\begin{array}{c}1.263^{* * *} \\
(0.118)\end{array}$ & $\begin{array}{c}-2.803^{* * *} \\
(0.423)\end{array}$ \\
\hline $\operatorname{lag} 5$ & $\begin{array}{c}0.281^{* * *} \\
(0.035)\end{array}$ & $\begin{array}{l}-0.032 \\
(0.058)\end{array}$ & $\begin{array}{c}0.043 \\
(0.071)\end{array}$ & $\begin{array}{l}-0.145^{*} \\
(0.084)\end{array}$ & $\begin{array}{c}0.003 \\
(0.097)\end{array}$ & $\begin{array}{c}-1.952^{* * *} \\
(0.392)\end{array}$ & $\begin{array}{c}-1.134^{* * *} \\
(0.300)\end{array}$ \\
\hline $\begin{array}{l}\text { Fixed effects } \\
\text { Item }\end{array}$ & Yes & Yes & Yes & Yes & Yes & Yes & Yes \\
\hline$R$-squared & 0 & 0 . & . & 0 . & 0.261 & 0.281 & 0.306 \\
\hline Observations & 45,507 & 42,473 & 39,439 & 36,405 & 33,371 & 30,337 & 27,303 \\
\hline
\end{tabular}

Note: The table presents the full estimation results of model (1) using the sales-weighted exchange $r a t e, ~ e r^{*}$, for $h=0,2, \cdots, 10,12$ and $L=5$. Robust standard errors (clustered by product) are in parentheses. The notation ${ }^{* * *}$ indicates significance at $1 \%$ level, ${ }^{* *}$ at $5 \%$ level, ${ }^{*}$ at $10 \%$ level. 
Table A4: Asymmetric ERPTs for imported products with $L=5$

\begin{tabular}{|c|c|c|c|c|c|c|c|}
\hline \multirow[b]{2}{*}{ Variable } & \multicolumn{7}{|c|}{ Horizon (h) } \\
\hline & 0 & 2 & 4 & 6 & 8 & 10 & 12 \\
\hline$\Delta \ln e r$ & $\begin{array}{l}-0.020^{*} \\
(0.012)\end{array}$ & $\begin{array}{c}-0.070 * * * \\
(0.023)\end{array}$ & $\begin{array}{c}0.108 * * * \\
(0.040)\end{array}$ & $\begin{array}{c}0.181 * * * \\
(0.043)\end{array}$ & $\begin{array}{c}0.161 * * * \\
(0.046)\end{array}$ & $\begin{array}{c}0.110^{* *} \\
(0.046)\end{array}$ & $\begin{array}{c}0.123^{* * *} \\
(0.042)\end{array}$ \\
\hline $\operatorname{lag} 1$ & $\begin{array}{c}0.037^{* * *} \\
(0.010)\end{array}$ & $\begin{array}{c}0.166^{* * *} \\
(0.014)\end{array}$ & $\begin{array}{c}0.221 * * * \\
(0.016)\end{array}$ & $\begin{array}{c}0.299 * * * \\
(0.018)\end{array}$ & $\begin{array}{c}0.321 * * * \\
(0.020)\end{array}$ & $\begin{array}{c}0.349^{* * *} * \\
(0.024)\end{array}$ & $\begin{array}{c}0.260^{* * *} \\
(0.022)\end{array}$ \\
\hline $\operatorname{lag} 2$ & $\begin{array}{c}0.104^{* * *} \\
(0.010)\end{array}$ & $\begin{array}{c}0.223^{* * *} \\
(0.012)\end{array}$ & $\begin{array}{c}0.247^{* * *} * \\
(0.012)\end{array}$ & $\begin{array}{c}0.294^{* * *} \\
(0.014)\end{array}$ & $\begin{array}{c}0.299^{* * *} \\
(0.015)\end{array}$ & $\begin{array}{c}0.244^{* * *} \\
(0.017)\end{array}$ & $\begin{array}{c}0.075^{* * *} \\
(0.018)\end{array}$ \\
\hline $\operatorname{lag} 3$ & $\begin{array}{c}0.056^{* * *} \\
(0.009)\end{array}$ & $\begin{array}{c}0.137^{* * *} \\
(0.013)\end{array}$ & $\begin{array}{c}0.227^{* * *} \\
(0.017)\end{array}$ & $\begin{array}{c}0.267 * * * \\
(0.016)\end{array}$ & $\begin{array}{c}0.370 * * * \\
(0.021)\end{array}$ & $\begin{array}{c}0.378^{* * *} * \\
(0.021)\end{array}$ & $\begin{array}{c}0.306^{* * *} \\
(0.022)\end{array}$ \\
\hline $\operatorname{lag} 4$ & $\begin{array}{c}0.055^{* * *} \\
(0.008)\end{array}$ & $\begin{array}{c}0.104^{* * *} \\
(0.010)\end{array}$ & $\begin{array}{c}0.151^{* * *} \\
(0.011)\end{array}$ & $\begin{array}{c}0.230^{* * *} \\
(0.012)\end{array}$ & $\begin{array}{c}0.236^{* * *} \\
(0.015)\end{array}$ & $\begin{array}{c}0.255^{* * *} \\
(0.019)\end{array}$ & $\begin{array}{c}0.297^{* * *} \\
(0.046)\end{array}$ \\
\hline $\operatorname{lag} 5$ & $\begin{array}{c}0.057^{* * *} \\
(0.009)\end{array}$ & $\begin{array}{c}0.149^{* * *} \\
(0.013)\end{array}$ & $\begin{array}{c}0.189^{* * *} \\
(0.013)\end{array}$ & $\begin{array}{c}0.228^{* * *} \\
(0.015)\end{array}$ & $\begin{array}{c}0.217^{* * *} \\
(0.016)\end{array}$ & $\begin{array}{c}0.072^{* * * *} \\
(0.014)\end{array}$ & $\begin{array}{c}-0.424^{* * *} \\
(0.047)\end{array}$ \\
\hline$\Delta \ln e r \times d$ & $\begin{array}{c}0.026 \\
(0.019)\end{array}$ & $\begin{array}{c}0.195^{* * *} \\
(0.043)\end{array}$ & $\begin{array}{c}0.187^{* * *} \\
(0.065)\end{array}$ & $\begin{array}{c}0.214^{* * *} \\
(0.068)\end{array}$ & $\begin{array}{c}0.444^{* * *} \\
(0.073)\end{array}$ & $\begin{array}{c}0.557^{* * *} \\
(0.076)\end{array}$ & $\begin{array}{c}0.648^{* * *} \\
(0.075)\end{array}$ \\
\hline \multicolumn{8}{|l|}{$\Delta \ln p$} \\
\hline $\operatorname{lag} 1$ & $\begin{array}{c}-0.213^{* * *} \\
(0.018)\end{array}$ & $\begin{array}{c}-0.344^{* * *} \\
(0.018)\end{array}$ & $\begin{array}{c}-0.417^{* * *} \\
(0.020)\end{array}$ & $\begin{array}{c}-0.588^{* * *} \\
(0.021)\end{array}$ & $\begin{array}{c}-0.708^{* * *} \\
(0.020)\end{array}$ & $\begin{array}{c}-0.793^{* * *} \\
(0.023)\end{array}$ & $\begin{array}{c}-0.804^{* * *} \\
(0.024)\end{array}$ \\
\hline $\operatorname{lag} 2$ & $\begin{array}{c}-0.127^{* * *} \\
(0.014)\end{array}$ & $\begin{array}{c}-0.205^{* * *} \\
(0.016)\end{array}$ & $\begin{array}{c}-0.332^{* * *} \\
(0.017)\end{array}$ & $\begin{array}{c}-0.532^{* * *} \\
(0.019)\end{array}$ & $\begin{array}{c}-0.636^{* * *} \\
(0.019)\end{array}$ & $\begin{array}{c}-0.729^{* * *} \\
(0.025)\end{array}$ & $\begin{array}{c}-0.736^{* * * *} \\
(0.026)\end{array}$ \\
\hline $\operatorname{lag} 3$ & $\begin{array}{c}-0.058^{* * *} \\
(0.011)\end{array}$ & $\begin{array}{c}-0.125^{* * *} \\
(0.016)\end{array}$ & $\begin{array}{c}-0.295^{* * *} \\
(0.019)\end{array}$ & $\begin{array}{c}-0.464^{* * *} \\
(0.018)\end{array}$ & $\begin{array}{c}-0.587^{* * *} \\
(0.020)\end{array}$ & $\begin{array}{c}-0.680^{* * *} \\
(0.023)\end{array}$ & $\begin{array}{c}-0.721^{* * * *} \\
(0.030)\end{array}$ \\
\hline $\operatorname{lag} 4$ & $\begin{array}{c}-0.025^{* *} \\
(0.011)\end{array}$ & $\begin{array}{c}-0.106^{* * *} \\
(0.015)\end{array}$ & $\begin{array}{c}-0.270^{* * *} \\
(0.018)\end{array}$ & $\begin{array}{c}-0.391^{* * *} \\
(0.018)\end{array}$ & $\begin{array}{c}-0.516^{* * *} \\
(0.018)\end{array}$ & $\begin{array}{c}-0.631^{* * *} \\
(0.022)\end{array}$ & $\begin{array}{c}-0.724^{* * * *} \\
(0.034)\end{array}$ \\
\hline $\operatorname{lag} 5$ & $\begin{array}{l}-0.011 \\
(0.010)\end{array}$ & $\begin{array}{c}-0.107^{* * * *} \\
(0.014)\end{array}$ & $\begin{array}{c}-0.226^{* * * *} \\
(0.016)\end{array}$ & $\begin{array}{c}-0.340^{* * * *} \\
(0.017)\end{array}$ & $\begin{array}{c}-0.434^{* * * *} \\
(0.018)\end{array}$ & $\begin{array}{c}-0.522^{* * *} \\
(0.024)\end{array}$ & $\begin{array}{c}-0.611^{* * *} \\
(0.037)\end{array}$ \\
\hline \multicolumn{8}{|l|}{$\Delta \ln q$} \\
\hline $\operatorname{lag} 1$ & & $\begin{array}{l}-0.002 \\
(0.002)\end{array}$ & $\begin{array}{c}-0.004^{*} \\
(0.002)\end{array}$ & $\begin{array}{l}-0.003 \\
(0.002)\end{array}$ & $\begin{array}{l}-0.003 \\
(0.002)\end{array}$ & $\begin{array}{c}0.000 \\
(0.002)\end{array}$ & $\begin{array}{l}0.004^{*} \\
(0.002)\end{array}$ \\
\hline $\operatorname{lag} 2$ & $\begin{array}{l}-0.002 \\
(0.001)\end{array}$ & $\begin{array}{l}-0.001 \\
(0.002)\end{array}$ & $\begin{array}{l}-0.002 \\
(0.002)\end{array}$ & $\begin{array}{l}-0.001 \\
(0.002)\end{array}$ & $\begin{array}{l}-0.001 \\
(0.002)\end{array}$ & $\begin{array}{c}0.003 \\
(0.002)\end{array}$ & $\begin{array}{c}0.006^{* *} \\
(0.002)\end{array}$ \\
\hline $\operatorname{lag} 3$ & $\begin{array}{l}-0.000 \\
(0.001)\end{array}$ & $\begin{array}{l}-0.001 \\
(0.002)\end{array}$ & $\begin{array}{l}-0.001 \\
(0.002)\end{array}$ & $\begin{array}{l}-0.001 \\
(0.002)\end{array}$ & $\begin{array}{c}0.002 \\
(0.002)\end{array}$ & $\begin{array}{c}0.005^{* *} \\
(0.003)\end{array}$ & $\begin{array}{c}0.005^{* *} \\
(0.002)\end{array}$ \\
\hline $\operatorname{lag} 4$ & $\begin{array}{c}0.000 \\
(0.001)\end{array}$ & $\begin{array}{l}-0.000 \\
(0.002)\end{array}$ & $\begin{array}{c}0.001 \\
(0.002)\end{array}$ & $\begin{array}{c}-0.001 \\
(0.002)\end{array}$ & $\begin{array}{c}0.003 \\
(0.002)\end{array}$ & $\begin{array}{c}0.004 \\
(0.003)\end{array}$ & $\begin{array}{c}0.011^{* * *} \\
(0.002)\end{array}$ \\
\hline $\operatorname{lag} 5$ & $\begin{array}{c}-0.001 \\
(0.001)\end{array}$ & $\begin{array}{c}0.000 \\
(0.001)\end{array}$ & $\begin{array}{l}-0.001 \\
(0.002)\end{array}$ & $\begin{array}{c}0.001 \\
(0.002)\end{array}$ & $\begin{array}{c}0.003 \\
(0.002)\end{array}$ & $\begin{array}{c}0.002 \\
(0.002)\end{array}$ & $\begin{array}{c}0.006^{* * *} \\
(0.002)\end{array}$ \\
\hline \multicolumn{8}{|l|}{$\Delta \ln C P I$} \\
\hline $\operatorname{lag} 1$ & $\begin{array}{c}0.805^{* * *} \\
(0.090)\end{array}$ & $\begin{array}{c}1.027^{* * *} \\
(0.158)\end{array}$ & $\begin{array}{c}1.536^{* * *} \\
(0.194)\end{array}$ & $\begin{array}{c}2.008^{* * *} \\
(0.234)\end{array}$ & $\begin{array}{c}3.122^{* * *} \\
(0.306)\end{array}$ & $\begin{array}{c}4.103^{* * *} \\
(0.441)\end{array}$ & $\begin{array}{c}3.164 * * * \\
(0.347)\end{array}$ \\
\hline $\operatorname{lag} 2$ & $\begin{array}{c}-0.093 \\
(0.093)\end{array}$ & $\begin{array}{c}0.063 \\
(0.131)\end{array}$ & $\begin{array}{c}-0.284^{*} \\
(0.152)\end{array}$ & $\begin{array}{l}-0.049 \\
(0.129)\end{array}$ & $\begin{array}{c}0.581^{* * *} \\
(0.195)\end{array}$ & $\begin{array}{c}0.726^{* * *} \\
(0.171)\end{array}$ & $\begin{array}{c}0.116 \\
(0.208)\end{array}$ \\
\hline $\operatorname{lag} 3$ & $\begin{array}{l}-0.048 \\
(0.104)\end{array}$ & $\begin{array}{l}-0.110 \\
(0.130)\end{array}$ & $\begin{array}{c}-0.513^{* * *} \\
(0.154)\end{array}$ & $\begin{array}{c}-0.482^{* * *} \\
(0.157)\end{array}$ & $\begin{array}{c}-0.347^{* * *} \\
(0.120)\end{array}$ & $\begin{array}{l}-0.175 \\
(0.145)\end{array}$ & $\begin{array}{c}-0.873^{* * *} \\
(0.134)\end{array}$ \\
\hline $\operatorname{lag} 4$ & $\begin{array}{c}0.126 \\
(0.090)\end{array}$ & $\begin{array}{c}-0.403^{* * *} \\
(0.121)\end{array}$ & $\begin{array}{c}-0.578^{* * *} \\
(0.142)\end{array}$ & $\begin{array}{c}-0.568^{* * *} \\
(0.169)\end{array}$ & $\begin{array}{c}-0.482^{* * *} \\
(0.166)\end{array}$ & $\begin{array}{c}-0.600^{* * *} \\
(0.161)\end{array}$ & $\begin{array}{c}-1.808^{* * * *} \\
(0.288)\end{array}$ \\
\hline $\operatorname{lag} 5$ & $\begin{array}{c}-0.819^{* * * *} \\
(0.089)\end{array}$ & $\begin{array}{c}-1.458^{* * * *} \\
(0.137)\end{array}$ & $\begin{array}{c}-1.079^{* * *} \\
(0.165)\end{array}$ & $\begin{array}{l}-0.303 \\
(0.210)\end{array}$ & $\begin{array}{c}0.854^{* * *} \\
(0.218)\end{array}$ & $\begin{array}{c}1.059^{* * *} \\
(0.228)\end{array}$ & $\begin{array}{l}0.348^{*} \\
(0.207)\end{array}$ \\
\hline $\begin{array}{l}\text { Fixed effects } \\
\text { Item }\end{array}$ & Yes & Yes & Yes & Yes & Yes & Yes & Yes \\
\hline$R$-squared & 0.092 & 0.219 & 0.338 & 0.436 & 0.496 & 0.518 & 0.541 \\
\hline Observations & 25,740 & 24,024 & 22,308 & 20,592 & 18,876 & 17,160 & 15,444 \\
\hline
\end{tabular}

Note: The table presents the full estimation results of model (2) for $h=0,2, \cdots, 10,12$ and $L=5$. Robust standard errors (clustered by product) are in parentheses. The notation $* * *$ indicates significance at $1 \%$ level, ${ }^{* *}$ at $5 \%$ level, $*$ at $10 \%$ level. 
Table A5: Asymmetric ERPTs for domestic products with $L=5$

\begin{tabular}{|c|c|c|c|c|c|c|c|}
\hline \multirow[b]{2}{*}{ Variable } & \multicolumn{7}{|c|}{ Horizon (h) } \\
\hline & 0 & 2 & 4 & 6 & 8 & 10 & 12 \\
\hline$\Delta \ln e r^{*}$ & $\begin{array}{c}-0.024^{* * *} \\
(0.008)\end{array}$ & $\begin{array}{c}-0.041^{* *} \\
(0.018)\end{array}$ & $\begin{array}{l}-0.012 \\
(0.023)\end{array}$ & $\begin{array}{c}0.097 * * * \\
(0.027)\end{array}$ & $\begin{array}{c}0.297^{* * *} \\
(0.032)\end{array}$ & $\begin{array}{c}0.340^{* * *} \\
(0.035)\end{array}$ & $\begin{array}{c}0.356^{* * *} \\
(0.031)\end{array}$ \\
\hline lag 1 & $\begin{array}{c}0.025^{* * *} * \\
(0.006)\end{array}$ & $\begin{array}{c}0.040^{* * *} \\
(0.007)\end{array}$ & $\begin{array}{c}0.106^{* * *} \\
(0.007)\end{array}$ & $\begin{array}{c}0.138^{* * *} \\
(0.009)\end{array}$ & $\begin{array}{c}0.110^{* * *} \\
(0.009)\end{array}$ & $\begin{array}{c}0.079 * * * \\
(0.010)\end{array}$ & $\begin{array}{c}0.023^{* * *} \\
(0.007)\end{array}$ \\
\hline $\operatorname{lag} 2$ & $\begin{array}{c}-0.030^{* * * *} \\
(0.006)\end{array}$ & $\begin{array}{c}-0.030^{* * *} \\
(0.010)\end{array}$ & $\begin{array}{c}-0.047^{* * *} \\
(0.011)\end{array}$ & $\begin{array}{c}0.006 \\
(0.013)\end{array}$ & $\begin{array}{l}-0.024 \\
(0.021)\end{array}$ & $\begin{array}{c}0.034 \\
(0.023)\end{array}$ & $\begin{array}{c}0.036 \\
(0.024)\end{array}$ \\
\hline $\operatorname{lag} 3$ & $\begin{array}{c}0.005 \\
(0.007)\end{array}$ & $\begin{array}{l}-0.001 \\
(0.009)\end{array}$ & $\begin{array}{c}0.055^{* * *} * \\
(0.013)\end{array}$ & $\begin{array}{c}0.109 * * * \\
(0.017)\end{array}$ & $\begin{array}{c}0.170^{* * *} \\
(0.025)\end{array}$ & $\begin{array}{c}0.160^{* * *} \\
(0.025)\end{array}$ & $\begin{array}{l}-0.031 \\
(0.021)\end{array}$ \\
\hline $\operatorname{lag} 4$ & $\begin{array}{c}-0.014^{* *} \\
(0.007)\end{array}$ & $\begin{array}{c}0.026^{* * *} \\
(0.008)\end{array}$ & $\begin{array}{c}0.113^{* * *} \\
(0.009)\end{array}$ & $\begin{array}{c}0.157^{* * *} \\
(0.012)\end{array}$ & $\begin{array}{c}0.210^{* * * *} \\
(0.015)\end{array}$ & $\begin{array}{c}0.066^{* * *} \\
(0.010)\end{array}$ & $\begin{array}{c}0.055^{* * * *} \\
(0.018)\end{array}$ \\
\hline $\operatorname{lag} 5$ & $\begin{array}{c}0.024^{* * *} \\
(0.005)\end{array}$ & $\begin{array}{c}0.101^{* * *} \\
(0.010)\end{array}$ & $\begin{array}{c}0.126^{* * *} \\
(0.015)\end{array}$ & $\begin{array}{c}0.130^{* * *} \\
(0.020)\end{array}$ & $\begin{array}{l}-0.024 \\
(0.024)\end{array}$ & $\begin{array}{c}-0.098^{* * *} \\
(0.021)\end{array}$ & $\begin{array}{c}-0.140 * * * \\
(0.036)\end{array}$ \\
\hline$\Delta \ln e r^{*} \times d$ & $\begin{array}{c}0.017 \\
(0.012)\end{array}$ & $\begin{array}{c}0.108^{* * *} \\
(0.028)\end{array}$ & $\begin{array}{c}0.089^{* *} \\
(0.035)\end{array}$ & $\begin{array}{c}0.057 \\
(0.043)\end{array}$ & $\begin{array}{c}-0.132^{* *} \\
(0.053)\end{array}$ & $\begin{array}{c}-0.214^{* * *} \\
(0.064)\end{array}$ & $\begin{array}{c}-0.296^{* * *} \\
(0.060)\end{array}$ \\
\hline \multicolumn{8}{|l|}{$\Delta \ln p$} \\
\hline $\operatorname{lag} 1$ & $\begin{array}{c}-0.131^{* * *} \\
(0.016)\end{array}$ & $\begin{array}{c}-0.290 * * * \\
(0.021)\end{array}$ & $\begin{array}{c}-0.358^{* * *} \\
(0.022)\end{array}$ & $\begin{array}{c}-0.477^{* * * *} \\
(0.021)\end{array}$ & $\begin{array}{c}-0.584^{* * *} \\
(0.021)\end{array}$ & $\begin{array}{c}-0.695^{* * * *} \\
(0.023)\end{array}$ & $\begin{array}{c}-0.736^{* * *} \\
(0.025)\end{array}$ \\
\hline $\operatorname{lag} 2$ & $\begin{array}{c}-0.126^{* * * *} \\
(0.012)\end{array}$ & $\begin{array}{c}-0.212^{* * *} \\
(0.017)\end{array}$ & $\begin{array}{c}-0.331^{* * *} \\
(0.020)\end{array}$ & $\begin{array}{c}-0.436^{* * *} \\
(0.020)\end{array}$ & $\begin{array}{c}-0.562^{* * *} \\
(0.023)\end{array}$ & $\begin{array}{c}-0.618^{* * *} \\
(0.028)\end{array}$ & $\begin{array}{c}-0.703^{* * *} \\
(0.029)\end{array}$ \\
\hline $\operatorname{lag} 3$ & $\begin{array}{c}-0.074^{* * *} \\
(0.008)\end{array}$ & $\begin{array}{c}-0.158^{* * *} \\
(0.015)\end{array}$ & $\begin{array}{c}-0.271^{* * *} \\
(0.017)\end{array}$ & $\begin{array}{c}-0.389^{* * * *} \\
(0.019)\end{array}$ & $\begin{array}{c}-0.493^{* * *} \\
(0.024)\end{array}$ & $\begin{array}{c}-0.542^{* * * *} \\
(0.027)\end{array}$ & $\begin{array}{c}-0.596^{* * *} \\
(0.033)\end{array}$ \\
\hline $\operatorname{lag} 4$ & $\begin{array}{c}-0.035^{* * * *} \\
(0.009)\end{array}$ & $\begin{array}{c}-0.132^{* * * *} \\
(0.015)\end{array}$ & $\begin{array}{c}-0.216^{* * * *} \\
(0.016)\end{array}$ & $\begin{array}{c}-0.324 * * * \\
(0.019)\end{array}$ & $\begin{array}{c}-0.408^{* * * *} \\
(0.025)\end{array}$ & $\begin{array}{c}-0.502^{* * *} \\
(0.029)\end{array}$ & $\begin{array}{c}-0.561^{* * *} \\
(0.034)\end{array}$ \\
\hline $\operatorname{lag} 5$ & $\begin{array}{c}-0.045^{* * *} \\
(0.009)\end{array}$ & $\begin{array}{c}-0.123^{* * *} \\
(0.012)\end{array}$ & $\begin{array}{c}-0.209^{* * *} \\
(0.016)\end{array}$ & $\begin{array}{c}-0.288^{* * *} \\
(0.020)\end{array}$ & $\begin{array}{c}-0.364^{* * *} \\
(0.025)\end{array}$ & $\begin{array}{c}-0.409^{* * *} \\
(0.030)\end{array}$ & $\begin{array}{c}-0.459^{* * *} \\
(0.033)\end{array}$ \\
\hline \multicolumn{8}{|l|}{$\Delta \ln q$} \\
\hline $\operatorname{lag} 1$ & & $\begin{array}{c}0.004^{* * *} \\
(0.001)\end{array}$ & $\begin{array}{c}0.003^{* *} \\
(0.001)\end{array}$ & $\begin{array}{c}0.002 \\
(0.002)\end{array}$ & $\begin{array}{c}0.001 \\
(0.002)\end{array}$ & $\begin{array}{c}0.007^{* * *} \\
(0.002)\end{array}$ & $\begin{array}{c}0.008^{* * *} \\
(0.003)\end{array}$ \\
\hline $\operatorname{lag} 2$ & $\begin{array}{c}0.003^{* * *} \\
(0.001)\end{array}$ & $\begin{array}{c}0.003^{* *} \\
(0.001)\end{array}$ & $\begin{array}{c}0.002 \\
(0.001)\end{array}$ & $\begin{array}{c}0.000 \\
(0.002)\end{array}$ & $\begin{array}{c}0.001 \\
(0.002)\end{array}$ & $\begin{array}{c}0.006^{* *} \\
(0.003)\end{array}$ & $\begin{array}{c}0.009 * * * \\
(0.003)\end{array}$ \\
\hline $\operatorname{lag} 3$ & $\begin{array}{c}0.001 \\
(0.001)\end{array}$ & $\begin{array}{c}0.001 \\
(0.001)\end{array}$ & $\begin{array}{l}-0.000 \\
(0.002)\end{array}$ & $\begin{array}{l}-0.002 \\
(0.002)\end{array}$ & $\begin{array}{c}0.000 \\
(0.002)\end{array}$ & $\begin{array}{c}0.004 \\
(0.003)\end{array}$ & $\begin{array}{c}0.008^{* *} \\
(0.003)\end{array}$ \\
\hline $\operatorname{lag} 4$ & $\begin{array}{c}0.000 \\
(0.001)\end{array}$ & $\begin{array}{c}0.001 \\
(0.001)\end{array}$ & $\begin{array}{l}-0.001 \\
(0.002)\end{array}$ & $\begin{array}{l}-0.000 \\
(0.002)\end{array}$ & $\begin{array}{l}-0.000 \\
(0.003)\end{array}$ & $\begin{array}{c}0.004 \\
(0.003)\end{array}$ & $\begin{array}{c}0.006^{* *} \\
(0.003)\end{array}$ \\
\hline $\operatorname{lag} 5$ & $\begin{array}{c}0.000 \\
(0.001)\end{array}$ & $\begin{array}{l}-0.001 \\
(0.001)\end{array}$ & $\begin{array}{c}-0.002^{*} \\
(0.001)\end{array}$ & $\begin{array}{c}0.001 \\
(0.002)\end{array}$ & $\begin{array}{c}0.000 \\
(0.002)\end{array}$ & $\begin{array}{l}0.004^{*} \\
(0.003)\end{array}$ & $\begin{array}{c}0.002 \\
(0.003)\end{array}$ \\
\hline \multicolumn{8}{|l|}{$\Delta \ln C P I$} \\
\hline $\operatorname{lag} 1$ & $\begin{array}{c}0.654^{* * *} \\
(0.044)\end{array}$ & $\begin{array}{c}0.883^{* * *} \\
(0.063)\end{array}$ & $\begin{array}{c}1.473^{* * *} \\
(0.091)\end{array}$ & $\begin{array}{c}0.915^{* * *} \\
(0.106)\end{array}$ & $\begin{array}{c}0.633^{* * *} \\
(0.149)\end{array}$ & $\begin{array}{c}-0.231 \\
(0.160)\end{array}$ & $\begin{array}{c}-0.400^{* *} \\
(0.164)\end{array}$ \\
\hline $\operatorname{lag} 2$ & $\begin{array}{c}-0.326^{* * *} \\
(0.054)\end{array}$ & $\begin{array}{c}0.384^{* * *} \\
(0.061)\end{array}$ & $\begin{array}{c}0.033 \\
(0.072)\end{array}$ & $\begin{array}{c}-0.408^{* * * *} \\
(0.084)\end{array}$ & $\begin{array}{c}-0.647^{* * *} \\
(0.111)\end{array}$ & $\begin{array}{c}-0.581^{* * * *} \\
(0.121)\end{array}$ & $\begin{array}{c}0.532^{* * *} \\
(0.116)\end{array}$ \\
\hline $\operatorname{lag} 3$ & $\begin{array}{c}0.632^{* * *} * \\
(0.060)\end{array}$ & $\begin{array}{c}0.590^{* * *} * \\
(0.057)\end{array}$ & $\begin{array}{c}0.225^{* * *} * \\
(0.077)\end{array}$ & $\begin{array}{c}-0.170^{* *} \\
(0.084)\end{array}$ & $\begin{array}{c}-0.663^{* * *} \\
(0.105)\end{array}$ & $\begin{array}{c}-0.078 \\
(0.093)\end{array}$ & $\begin{array}{c}0.301 \\
(0.293)\end{array}$ \\
\hline $\operatorname{lag} 4$ & $\begin{array}{l}-0.068 \\
(0.049)\end{array}$ & $\begin{array}{l}-0.106 \\
(0.065)\end{array}$ & $\begin{array}{c}-0.609 * * * \\
(0.081)\end{array}$ & $\begin{array}{c}-0.465^{* * *} \\
(0.098)\end{array}$ & $\begin{array}{c}0.349^{* * *} \\
(0.109)\end{array}$ & $\begin{array}{c}1.277^{* * *} \\
(0.117)\end{array}$ & $\begin{array}{c}-1.714^{* * *} \\
(0.548)\end{array}$ \\
\hline $\operatorname{lag} 5$ & $\begin{array}{c}0.289^{* * *} \\
(0.036)\end{array}$ & $\begin{array}{c}0.025 \\
(0.058)\end{array}$ & $\begin{array}{c}0.094 \\
(0.069)\end{array}$ & $\begin{array}{l}-0.108 \\
(0.086)\end{array}$ & $\begin{array}{l}-0.091 \\
(0.112)\end{array}$ & $\begin{array}{c}-3.020^{* * *} \\
(0.456)\end{array}$ & $\begin{array}{c}-2.801^{* * * *} \\
(0.513)\end{array}$ \\
\hline $\begin{array}{l}\text { Fixed effects } \\
\text { Item }\end{array}$ & Yes & Yes & Yes & Yes & Yes & Yes & Yes \\
\hline$R$-squared & 0.065 & 0.139 & 0.193 & 0.238 & 0.262 & 0.283 & 0.310 \\
\hline Observations & 45,507 & 42,473 & 39,439 & 36,405 & 33,371 & 30,337 & 27,303 \\
\hline
\end{tabular}

Note: The table presents the full estimation results of model (2) using the sales-weighted exchange rate, $\mathrm{er}^{*}$, for $h=0,2, \cdots, 10,12$ and $L=5$. Robust standard errors (clustered by product) are in parentheses. The notation ${ }^{* * *}$ indicates significance at $1 \%$ level, ${ }^{* *}$ at $5 \%$ level, ${ }^{*}$ at $10 \%$ level. 
Table A6: Large vs small asymmetric ERPTs: imported products with $L=5$

\begin{tabular}{|c|c|c|c|c|c|c|c|}
\hline \multirow[b]{2}{*}{ Variable } & \multicolumn{7}{|c|}{ Horizon (h) } \\
\hline & 0 & 2 & 4 & 6 & 8 & 10 & 12 \\
\hline$\Delta \ln e r$ & $\begin{array}{c}0.032 \\
(0.028)\end{array}$ & $\begin{array}{c}0.088 \\
(0.054)\end{array}$ & $\begin{array}{c}0.078 \\
(0.073)\end{array}$ & $\begin{array}{c}0.348^{* * *} \\
(0.092)\end{array}$ & $\begin{array}{c}0.393^{* * *} \\
(0.103)\end{array}$ & $\begin{array}{c}0.639 * * * \\
(0.104)\end{array}$ & $\begin{array}{c}1.034^{* * *} \\
(0.129)\end{array}$ \\
\hline $\operatorname{lag} 1$ & $\begin{array}{c}0.038^{* * *} \\
(0.010)\end{array}$ & $\begin{array}{c}0.165^{* * *} \\
(0.014)\end{array}$ & $\begin{array}{c}0.210^{* * *} \\
(0.015)\end{array}$ & $\begin{array}{c}0.282^{* * *} \\
(0.017)\end{array}$ & $\begin{array}{c}0.268^{* * *} \\
(0.015)\end{array}$ & $\begin{array}{c}0.266^{* * *} \\
(0.018)\end{array}$ & $\begin{array}{c}0.189^{* * * *} \\
(0.018)\end{array}$ \\
\hline $\operatorname{lag} 2$ & $\begin{array}{c}0.105 * * * \\
(0.010)\end{array}$ & $\begin{array}{c}0.223^{* * *} * \\
(0.012)\end{array}$ & $\begin{array}{c}0.237 * * * \\
(0.011)\end{array}$ & $\begin{array}{c}0.275^{* * *} * \\
(0.013)\end{array}$ & $\begin{array}{c}0.257^{* * *} \\
(0.012)\end{array}$ & $\begin{array}{c}0.204^{* * *} \\
(0.015)\end{array}$ & $\begin{array}{c}0.023 \\
(0.021)\end{array}$ \\
\hline $\operatorname{lag} 3$ & $\begin{array}{c}0.055^{* * *} \\
(0.009)\end{array}$ & $\begin{array}{c}0.135^{* * *} \\
(0.013)\end{array}$ & $\begin{array}{c}0.234^{* * *} \\
(0.018)\end{array}$ & $\begin{array}{c}0.273^{* * *} \\
(0.017)\end{array}$ & $\begin{array}{c}0.399 * * * \\
(0.025)\end{array}$ & $\begin{array}{c}0.383^{* * *} \\
(0.023)\end{array}$ & $\begin{array}{c}0.288^{* * *} \\
(0.026)\end{array}$ \\
\hline $\operatorname{lag} 4$ & $\begin{array}{c}0.059 * * * \\
(0.008)\end{array}$ & $\begin{array}{c}0.113^{* * *} \\
(0.010)\end{array}$ & $\begin{array}{c}0.143^{* * *} \\
(0.011)\end{array}$ & $\begin{array}{c}0.230 * * * \\
(0.012)\end{array}$ & $\begin{array}{c}0.215^{* * *} \\
(0.013)\end{array}$ & $\begin{array}{c}0.220^{* * *} \\
(0.016)\end{array}$ & $\begin{array}{c}0.386 * * * \\
(0.052)\end{array}$ \\
\hline $\operatorname{lag} 5$ & $\begin{array}{c}0.058^{* * *} \\
(0.009)\end{array}$ & $\begin{array}{c}0.155^{* * *} \\
(0.014)\end{array}$ & $\begin{array}{c}0.200^{* * *} \\
(0.013)\end{array}$ & $\begin{array}{c}0.243^{* * *} \\
(0.016)\end{array}$ & $\begin{array}{c}0.258^{* * *} \\
(0.019)\end{array}$ & $\begin{array}{c}0.142^{* * *} \\
(0.018)\end{array}$ & $\begin{array}{c}-0.427^{* * *} \\
(0.064)\end{array}$ \\
\hline$\Delta \ln e r \times d$ & $\begin{array}{l}-0.042 \\
(0.044)\end{array}$ & $\begin{array}{c}0.024 \\
(0.088)\end{array}$ & $\begin{array}{c}0.361^{* * *} \\
(0.122)\end{array}$ & $\begin{array}{l}0.228^{*} \\
(0.136)\end{array}$ & $\begin{array}{c}0.719^{* * *} \\
(0.164)\end{array}$ & $\begin{array}{c}0.515^{* * *} \\
(0.169)\end{array}$ & $\begin{array}{c}0.059 \\
(0.171)\end{array}$ \\
\hline$\Delta \ln e r \times$ Over $10 \%$ & $\begin{array}{c}-0.059 * * \\
(0.024)\end{array}$ & $\begin{array}{c}-0.176^{* * *} \\
(0.045)\end{array}$ & $\begin{array}{c}0.026 \\
(0.053)\end{array}$ & $\begin{array}{c}-0.183^{* * *} \\
(0.062)\end{array}$ & $\begin{array}{c}-0.252^{* * *} \\
(0.068)\end{array}$ & $\begin{array}{c}-0.473^{* * *} \\
(0.071)\end{array}$ & $\begin{array}{c}-0.812^{* * *} \\
(0.093)\end{array}$ \\
\hline$\Delta \ln e r \times d \times$ Over $10 \%$ & $\begin{array}{c}0.069^{*} \\
(0.036)\end{array}$ & $\begin{array}{c}0.174^{* * *} \\
(0.058)\end{array}$ & $\begin{array}{c}-0.172^{* *} \\
(0.069)\end{array}$ & $\begin{array}{l}-0.019 \\
(0.075)\end{array}$ & $\begin{array}{c}-0.279^{* * *} \\
(0.093)\end{array}$ & $\begin{array}{l}-0.083 \\
(0.107)\end{array}$ & $\begin{array}{c}0.372^{* * *} \\
(0.124)\end{array}$ \\
\hline $\begin{array}{l}\Delta \ln p \\
\quad \operatorname{lag} 1\end{array}$ & $\begin{array}{c}-0.214^{* * *} \\
(0.018)\end{array}$ & $\begin{array}{c}-0.348^{* * *} \\
(0.018)\end{array}$ & $\begin{array}{c}-0.417^{* * *} \\
(0.020)\end{array}$ & $\begin{array}{c}-0.592^{* * *} \\
(0.021)\end{array}$ & $\begin{array}{c}-0.715^{* * *} \\
(0.020)\end{array}$ & $\begin{array}{c}-0.792^{* * *} \\
(0.023)\end{array}$ & $\begin{array}{c}-0.802^{* * *} \\
(0.024)\end{array}$ \\
\hline $\operatorname{lag} 2$ & $\begin{array}{c}-0.129^{* * *} \\
(0.014)\end{array}$ & $\begin{array}{c}-0.209^{* * *} \\
(0.016)\end{array}$ & $\begin{array}{c}-0.333^{* * *} \\
(0.017)\end{array}$ & $\begin{array}{c}-0.539^{* * *} \\
(0.020)\end{array}$ & $\begin{array}{c}-0.648^{* * *} \\
(0.020)\end{array}$ & $\begin{array}{c}-0.730^{* * *} \\
(0.025)\end{array}$ & $\begin{array}{c}-0.737^{* * *} \\
(0.026)\end{array}$ \\
\hline $\operatorname{lag} 3$ & $\begin{array}{c}-0.060 * * * \\
(0.011)\end{array}$ & $\begin{array}{c}-0.130 * * * \\
(0.017)\end{array}$ & $\begin{array}{c}-0.294^{* * *} \\
(0.019)\end{array}$ & $\begin{array}{c}-0.468^{* * *} \\
(0.019)\end{array}$ & $\begin{array}{c}-0.589 * * * \\
(0.021)\end{array}$ & $\begin{array}{c}-0.669^{* * * *} \\
(0.024)\end{array}$ & $\begin{array}{c}-0.708^{* * *} * \\
(0.031)\end{array}$ \\
\hline $\operatorname{lag} 4$ & $\begin{array}{c}-0.026^{* *} \\
(0.011)\end{array}$ & $\begin{array}{c}-0.109 * * * \\
(0.015)\end{array}$ & $\begin{array}{c}-0.270^{* * *} \\
(0.018)\end{array}$ & $\begin{array}{c}-0.393 * * * \\
(0.019)\end{array}$ & $\begin{array}{c}-0.519 * * * \\
(0.019)\end{array}$ & $\begin{array}{c}-0.614^{* * *} \\
(0.022)\end{array}$ & $\begin{array}{c}-0.698^{* * *} * \\
(0.035)\end{array}$ \\
\hline $\operatorname{lag} 5$ & $\begin{array}{c}-0.011 \\
(0.010)\end{array}$ & $\begin{array}{c}-0.107^{* * *} \\
(0.014)\end{array}$ & $\begin{array}{c}-0.227^{* * *} \\
(0.016)\end{array}$ & $\begin{array}{c}-0.339^{* * *} \\
(0.017)\end{array}$ & $\begin{array}{c}-0.431^{* * *} \\
(0.019)\end{array}$ & $\begin{array}{c}-0.499^{* * *} \\
(0.024)\end{array}$ & $\begin{array}{c}-0.575^{* * *} \\
(0.037)\end{array}$ \\
\hline$\Delta \ln q$ & & & & & & & \\
\hline lag 1 & & $\begin{array}{c}-0.003^{*} \\
(0.002)\end{array}$ & $\begin{array}{c}-0.004^{*} \\
(0.002)\end{array}$ & $\begin{array}{c}-0.004^{*} \\
(0.002)\end{array}$ & $\begin{array}{l}-0.003 \\
(0.002)\end{array}$ & $\begin{array}{c}0.000 \\
(0.002)\end{array}$ & $\begin{array}{l}0.004^{*} \\
(0.002)\end{array}$ \\
\hline $\operatorname{lag} 2$ & $\begin{array}{c}-0.002^{*} \\
(0.001)\end{array}$ & $\begin{array}{l}-0.002 \\
(0.002)\end{array}$ & $\begin{array}{l}-0.002 \\
(0.002)\end{array}$ & $\begin{array}{l}-0.001 \\
(0.002)\end{array}$ & $\begin{array}{l}-0.001 \\
(0.002)\end{array}$ & $\begin{array}{c}0.003 \\
(0.002)\end{array}$ & $\begin{array}{c}0.005^{* *} \\
(0.002)\end{array}$ \\
\hline $\operatorname{lag} 3$ & $\begin{array}{l}-0.000 \\
(0.001)\end{array}$ & $\begin{array}{l}-0.002 \\
(0.002)\end{array}$ & $\begin{array}{l}-0.000 \\
(0.002)\end{array}$ & $\begin{array}{l}-0.001 \\
(0.002)\end{array}$ & $\begin{array}{c}0.002 \\
(0.002)\end{array}$ & $\begin{array}{l}0.005^{* *} \\
(0.002)\end{array}$ & $\begin{array}{c}0.004^{* *} \\
(0.002)\end{array}$ \\
\hline $\operatorname{lag} 4$ & $\begin{array}{c}0.000 \\
(0.001)\end{array}$ & $\begin{array}{l}-0.001 \\
(0.002)\end{array}$ & $\begin{array}{c}0.001 \\
(0.002)\end{array}$ & $\begin{array}{l}-0.001 \\
(0.002)\end{array}$ & $\begin{array}{l}0.004^{*} \\
(0.002)\end{array}$ & $\begin{array}{l}0.005^{*} \\
(0.003)\end{array}$ & $\begin{array}{c}0.012^{* * *} \\
(0.002)\end{array}$ \\
\hline $\operatorname{lag} 5$ & $\begin{array}{l}-0.001 \\
(0.001)\end{array}$ & $\begin{array}{c}0.000 \\
(0.001)\end{array}$ & $\begin{array}{l}-0.001 \\
(0.002)\end{array}$ & $\begin{array}{c}0.001 \\
(0.002)\end{array}$ & $\begin{array}{c}0.002 \\
(0.002)\end{array}$ & $\begin{array}{c}0.001 \\
(0.002)\end{array}$ & $\begin{array}{c}0.005^{* * * *} \\
(0.002)\end{array}$ \\
\hline$\Delta \ln C P I$ & & & & & & & \\
\hline $\operatorname{lag} 1$ & $\begin{array}{c}0.797^{* * *} \\
(0.091)\end{array}$ & $\begin{array}{c}1.016^{* * *} \\
(0.165)\end{array}$ & $\begin{array}{c}1.611^{* * *} \\
(0.211)\end{array}$ & $\begin{array}{c}2.063^{* * *} \\
(0.257)\end{array}$ & $\begin{array}{c}3.322^{* * *} \\
(0.358)\end{array}$ & $\begin{array}{c}4.127^{* * *} \\
(0.473)\end{array}$ & $\begin{array}{c}2.958^{* * * *} \\
(0.331)\end{array}$ \\
\hline $\operatorname{lag} 2$ & $\begin{array}{l}-0.094 \\
(0.095)\end{array}$ & $\begin{array}{l}-0.006 \\
(0.114)\end{array}$ & $\begin{array}{c}-0.552^{* * *} \\
(0.156)\end{array}$ & $\begin{array}{c}-0.555^{* * * *} \\
(0.184)\end{array}$ & $\begin{array}{c}-0.677^{* *} \\
(0.285)\end{array}$ & $\begin{array}{c}-1.064^{* * * *} \\
(0.320)\end{array}$ & $\begin{array}{c}-1.839^{* * * *} \\
(0.528)\end{array}$ \\
\hline $\operatorname{lag} 3$ & $\begin{array}{l}-0.026 \\
(0.112)\end{array}$ & $\begin{array}{l}-0.008 \\
(0.154)\end{array}$ & $\begin{array}{c}-0.348^{* *} \\
(0.166)\end{array}$ & $\begin{array}{l}-0.105 \\
(0.168)\end{array}$ & $\begin{array}{c}0.606^{* * *} \\
(0.200)\end{array}$ & $\begin{array}{c}1.177^{* * *} \\
(0.205)\end{array}$ & $\begin{array}{c}0.910^{* * * *} \\
(0.264)\end{array}$ \\
\hline $\operatorname{lag} 4$ & $\begin{array}{c}0.090 \\
(0.086)\end{array}$ & $\begin{array}{c}-0.540^{* * *} \\
(0.111)\end{array}$ & $\begin{array}{c}-0.654^{* * *} \\
(0.138)\end{array}$ & $\begin{array}{c}-0.913^{* * *} \\
(0.220)\end{array}$ & $\begin{array}{c}-1.273^{* * *} \\
(0.250)\end{array}$ & $\begin{array}{c}-1.924^{* * *} \\
(0.290)\end{array}$ & $\begin{array}{c}-3.466^{* * *} \\
(0.493)\end{array}$ \\
\hline $\operatorname{lag} 5$ & $\begin{array}{c}-0.714^{* * *} \\
(0.101)\end{array}$ & $\begin{array}{c}-1.118^{* * *} \\
(0.157)\end{array}$ & $\begin{array}{c}-1.033^{* * *} \\
(0.175)\end{array}$ & $\begin{array}{c}0.206 \\
(0.268)\end{array}$ & $\begin{array}{c}1.798^{* * *} \\
(0.323)\end{array}$ & $\begin{array}{c}2.665^{* * *} \\
(0.415)\end{array}$ & $\begin{array}{c}2.144^{* * *} \\
(0.396)\end{array}$ \\
\hline $\begin{array}{l}\text { Fixed effects } \\
\text { Item }\end{array}$ & Yes & Yes & Yes & Yes & Yes & Yes & Yes \\
\hline $\begin{array}{l}R \text {-squared } \\
\text { Observations }\end{array}$ & $\begin{array}{c}0.092 \\
25,740\end{array}$ & $\begin{array}{c}0.221 \\
24,024\end{array}$ & $\begin{array}{c}0.339 \\
22,308\end{array}$ & $\begin{array}{c}0.438 \\
20,592\end{array}$ & $\begin{array}{c}0.504 \\
18,876\end{array}$ & $\begin{array}{c}0.527 \\
17,160\end{array}$ & $\begin{array}{c}0.555 \\
15,444\end{array}$ \\
\hline
\end{tabular}

Note: The table presents the full estimation results of model (??) for $h=0,2, \cdots, 10,12$ and $L=5$. Robust standard errors (clustered by product) are in parentheses. The notation $* * *$ indicates significance at $1 \%$ level, ${ }^{* *}$ at $5 \%$ level, ${ }^{*}$ at $10 \%$ level. 
Table A7: Large vs small asymmetric ERPTs: domestic products with $L=5$

\begin{tabular}{|c|c|c|c|c|c|c|c|}
\hline \multirow[b]{2}{*}{ Variable } & \multicolumn{7}{|c|}{ "Horizon $(\mathrm{h})$} \\
\hline & 0 & 2 & 4 & 6 & 8 & 10 & 12 \\
\hline$\Delta \ln e r^{*}$ & $\begin{array}{c}0.000 \\
(0.014)\end{array}$ & $\begin{array}{c}0.093^{* * *} \\
(0.033)\end{array}$ & $\begin{array}{c}0.112^{* *} \\
(0.045)\end{array}$ & $\begin{array}{c}0.457^{* * *} \\
(0.048)\end{array}$ & $\begin{array}{c}0.649^{* * *} \\
(0.051)\end{array}$ & $\begin{array}{c}0.713^{* * *} \\
(0.060)\end{array}$ & $\begin{array}{c}0.750^{* * *} \\
(0.057)\end{array}$ \\
\hline $\operatorname{lag} 1$ & $\begin{array}{c}0.028 * * * \\
(0.006)\end{array}$ & $\begin{array}{c}0.051^{* * *} \\
(0.006)\end{array}$ & $\begin{array}{c}0.117^{* * *} \\
(0.007)\end{array}$ & $\begin{array}{c}0.162^{* * *} \\
(0.009)\end{array}$ & $\begin{array}{c}0.140 * * * \\
(0.009)\end{array}$ & $\begin{array}{c}0.119^{* * *} \\
(0.011)\end{array}$ & $\begin{array}{c}0.064 * * * \\
(0.011)\end{array}$ \\
\hline $\operatorname{lag} 2$ & $\begin{array}{c}-0.033^{* * *} \\
(0.006)\end{array}$ & $\begin{array}{c}-0.040^{* * *} \\
(0.010)\end{array}$ & $\begin{array}{c}-0.063^{* * *} \\
(0.011)\end{array}$ & $\begin{array}{l}-0.009 \\
(0.013)\end{array}$ & $\begin{array}{c}-0.032 \\
(0.020)\end{array}$ & $\begin{array}{l}0.038^{*} \\
(0.021)\end{array}$ & $\begin{array}{c}0.030 \\
(0.021)\end{array}$ \\
\hline $\operatorname{lag} 3$ & $\begin{array}{c}0.007 \\
(0.007)\end{array}$ & $\begin{array}{c}0.011 \\
(0.009)\end{array}$ & $\begin{array}{c}0.070^{* * *} \\
(0.014)\end{array}$ & $\begin{array}{c}0.138^{* * *} \\
(0.017)\end{array}$ & $\begin{array}{c}0.180^{* * *} * \\
(0.025)\end{array}$ & $\begin{array}{c}0.132^{* * *} * \\
(0.027)\end{array}$ & $\begin{array}{c}-0.055^{* *} \\
(0.025)\end{array}$ \\
\hline $\operatorname{lag} 4$ & $\begin{array}{l}-0.011 \\
(0.007)\end{array}$ & $\begin{array}{c}0.045^{* * *} \\
(0.009)\end{array}$ & $\begin{array}{c}0.129^{* * *} \\
(0.010)\end{array}$ & $\begin{array}{c}0.211^{* * *} \\
(0.013)\end{array}$ & $\begin{array}{c}0.279^{* * *} \\
(0.018)\end{array}$ & $\begin{array}{c}0.176^{* * *} * \\
(0.017)\end{array}$ & $\begin{array}{c}0.175^{* * *} \\
(0.019)\end{array}$ \\
\hline $\operatorname{lag} 5$ & $\begin{array}{c}0.017^{* * *} \\
(0.005)\end{array}$ & $\begin{array}{c}0.070^{* * *} \\
(0.009)\end{array}$ & $\begin{array}{c}0.088^{* * * *} \\
(0.014)\end{array}$ & $\begin{array}{c}0.074^{* * *} \\
(0.019)\end{array}$ & $\begin{array}{c}-0.080^{* * *} \\
(0.023)\end{array}$ & $\begin{array}{c}-0.182^{* * *} \\
(0.018)\end{array}$ & $\begin{array}{c}-0.237^{* * *} \\
(0.029)\end{array}$ \\
\hline$\Delta \ln e r^{*} \times d$ & $\begin{array}{c}-0.048^{* *} \\
(0.023)\end{array}$ & $\begin{array}{c}-0.181^{* * *} \\
(0.052)\end{array}$ & $\begin{array}{c}-0.243^{* * *} \\
(0.071)\end{array}$ & $\begin{array}{c}-0.562^{* * *} \\
(0.085)\end{array}$ & $\begin{array}{c}-0.744^{* * *} \\
(0.102)\end{array}$ & $\begin{array}{c}-0.948^{* * *} \\
(0.139)\end{array}$ & $\begin{array}{c}-1.058^{* * *} \\
(0.130)\end{array}$ \\
\hline$\Delta \ln e^{*} \times$ Over $10 \%$ & $\begin{array}{c}-0.026^{* *} \\
(0.013)\end{array}$ & $\begin{array}{c}-0.148^{* * *} \\
(0.028)\end{array}$ & $\begin{array}{c}-0.124^{* * *} \\
(0.034)\end{array}$ & $\begin{array}{c}-0.381 * * * \\
(0.031)\end{array}$ & $\begin{array}{c}-0.359^{* * *} \\
(0.032)\end{array}$ & $\begin{array}{c}-0.375^{* * *} \\
(0.034)\end{array}$ & $\begin{array}{c}-0.382^{* * *} \\
(0.041)\end{array}$ \\
\hline$\Delta \ln e r^{*} \times d \times$ Over $10 \%$ & $\begin{array}{c}0.069^{* * *} \\
(0.020)\end{array}$ & $\begin{array}{c}0.307^{* * *} \\
(0.036)\end{array}$ & $\begin{array}{c}0.343^{* * *} \\
(0.047)\end{array}$ & $\begin{array}{c}0.620^{* * *} \\
(0.053)\end{array}$ & $\begin{array}{c}0.584^{* * *} \\
(0.063)\end{array}$ & $\begin{array}{c}0.689^{* * * *} \\
(0.084)\end{array}$ & $\begin{array}{c}0.696^{* * *} \\
(0.077)\end{array}$ \\
\hline \multicolumn{8}{|l|}{$\Delta \ln p$} \\
\hline $\operatorname{lag} 1$ & $\begin{array}{c}-0.131^{* * *} \\
(0.016)\end{array}$ & $\begin{array}{c}-0.292^{* * *} \\
(0.021)\end{array}$ & $\begin{array}{c}-0.360^{* * *} \\
(0.022)\end{array}$ & $\begin{array}{c}-0.482^{* * *} \\
(0.021)\end{array}$ & $\begin{array}{c}-0.585^{* * *} \\
(0.021)\end{array}$ & $\begin{array}{c}-0.696^{* * *} \\
(0.022)\end{array}$ & $\begin{array}{c}-0.733^{* * *} \\
(0.024)\end{array}$ \\
\hline $\operatorname{lag} 2$ & $\begin{array}{c}-0.126^{* * *} \\
(0.012)\end{array}$ & $\begin{array}{c}-0.213^{* * *} \\
(0.017)\end{array}$ & $\begin{array}{c}-0.333^{* * *} \\
(0.020)\end{array}$ & $\begin{array}{c}-0.443^{* * *} \\
(0.020)\end{array}$ & $\begin{array}{c}-0.561^{* * *} \\
(0.023)\end{array}$ & $\begin{array}{c}-0.620^{* * *} \\
(0.028)\end{array}$ & $\begin{array}{c}-0.703^{* * *} \\
(0.029)\end{array}$ \\
\hline $\operatorname{lag} 3$ & $\begin{array}{c}-0.074^{* * *} \\
(0.008)\end{array}$ & $\begin{array}{c}-0.160^{* * *} \\
(0.015)\end{array}$ & $\begin{array}{c}-0.274^{* * *} \\
(0.017)\end{array}$ & $\begin{array}{c}-0.401 * * * \\
(0.020)\end{array}$ & $\begin{array}{c}-0.493^{* * *} \\
(0.023)\end{array}$ & $\begin{array}{c}-0.543^{* * *} \\
(0.027)\end{array}$ & $\begin{array}{c}-0.592^{* * *} \\
(0.033)\end{array}$ \\
\hline $\operatorname{lag} 4$ & $\begin{array}{c}-0.035^{* * *} \\
(0.009)\end{array}$ & $\begin{array}{c}-0.132^{* * *} \\
(0.015)\end{array}$ & $\begin{array}{c}-0.217^{* * *} \\
(0.017)\end{array}$ & $\begin{array}{c}-0.325^{* * *} \\
(0.019)\end{array}$ & $\begin{array}{c}-0.403^{* * *} \\
(0.025)\end{array}$ & $\begin{array}{c}-0.495^{* * *} \\
(0.028)\end{array}$ & $\begin{array}{c}-0.550^{* * *} * \\
(0.033)\end{array}$ \\
\hline $\operatorname{lag} 5$ & $\begin{array}{c}-0.044^{* * *} \\
(0.009)\end{array}$ & $\begin{array}{c}-0.121^{* * *} \\
(0.013)\end{array}$ & $\begin{array}{c}-0.207^{* * *} \\
(0.016)\end{array}$ & $\begin{array}{c}-0.279^{* * *} \\
(0.020)\end{array}$ & $\begin{array}{c}-0.353^{* * *} \\
(0.025)\end{array}$ & $\begin{array}{c}-0.397^{* * *} \\
(0.030)\end{array}$ & $\begin{array}{c}-0.441^{* * *} \\
(0.033)\end{array}$ \\
\hline \multicolumn{8}{|l|}{$\Delta \ln q$} \\
\hline $\operatorname{lag} 1$ & & $\begin{array}{c}0.004^{* * *} \\
(0.001)\end{array}$ & $\begin{array}{c}0.003^{* *} \\
(0.001)\end{array}$ & $\begin{array}{c}0.002 \\
(0.002)\end{array}$ & $\begin{array}{c}0.001 \\
(0.002)\end{array}$ & $\begin{array}{c}0.006^{* * *} \\
(0.002)\end{array}$ & $\begin{array}{c}0.007 * * * \\
(0.003)\end{array}$ \\
\hline $\operatorname{lag} 2$ & $\begin{array}{c}0.003^{* * *} \\
(0.001)\end{array}$ & $\begin{array}{c}0.003^{* *} \\
(0.001)\end{array}$ & $\begin{array}{c}0.002 \\
(0.001)\end{array}$ & $\begin{array}{l}-0.000 \\
(0.002)\end{array}$ & $\begin{array}{c}0.001 \\
(0.002)\end{array}$ & $\begin{array}{c}0.005^{* *} \\
(0.003)\end{array}$ & $\begin{array}{c}0.008 * * * \\
(0.003)\end{array}$ \\
\hline $\operatorname{lag} 3$ & $\begin{array}{c}0.001 \\
(0.001)\end{array}$ & $\begin{array}{c}0.001 \\
(0.001)\end{array}$ & $\begin{array}{l}-0.000 \\
(0.002)\end{array}$ & $\begin{array}{l}-0.002 \\
(0.002)\end{array}$ & $\begin{array}{c}0.000 \\
(0.002)\end{array}$ & $\begin{array}{c}0.004 \\
(0.003)\end{array}$ & $\begin{array}{c}0.008^{* *} \\
(0.003)\end{array}$ \\
\hline $\operatorname{lag} 4$ & $\begin{array}{c}0.000 \\
(0.001)\end{array}$ & $\begin{array}{c}0.001 \\
(0.001)\end{array}$ & $\begin{array}{l}-0.001 \\
(0.002)\end{array}$ & $\begin{array}{l}-0.000 \\
(0.002)\end{array}$ & $\begin{array}{l}-0.000 \\
(0.003)\end{array}$ & $\begin{array}{c}0.004 \\
(0.003)\end{array}$ & $\begin{array}{c}0.006^{* *} \\
(0.003)\end{array}$ \\
\hline $\operatorname{lag} 5$ & $\begin{array}{c}0.000 \\
(0.001)\end{array}$ & $\begin{array}{l}-0.001 \\
(0.001)\end{array}$ & $\begin{array}{l}-0.002 \\
(0.001)\end{array}$ & $\begin{array}{c}0.001 \\
(0.002)\end{array}$ & $\begin{array}{c}0.001 \\
(0.002)\end{array}$ & $\begin{array}{l}0.005^{*} \\
(0.003)\end{array}$ & $\begin{array}{c}0.002 \\
(0.003)\end{array}$ \\
\hline \multicolumn{8}{|l|}{$\Delta \ln C P I$} \\
\hline $\operatorname{lag} 1$ & $\begin{array}{c}0.708^{* * *} \\
(0.051)\end{array}$ & $\begin{array}{c}1.069^{* * *} \\
(0.077)\end{array}$ & $\begin{array}{c}1.760^{* * *} \\
(0.105)\end{array}$ & $\begin{array}{c}1.120^{* * *} \\
(0.110)\end{array}$ & $\begin{array}{c}0.772^{* * *} \\
(0.140)\end{array}$ & $\begin{array}{l}-0.117 \\
(0.125)\end{array}$ & $\begin{array}{c}-0.245^{* *} \\
(0.110)\end{array}$ \\
\hline $\operatorname{lag} 2$ & $\begin{array}{c}-0.377^{* * * *} \\
(0.054)\end{array}$ & $\begin{array}{c}0.178^{* * *} \\
(0.060)\end{array}$ & $\begin{array}{c}-0.243^{* * *} \\
(0.080)\end{array}$ & $\begin{array}{c}-0.785^{* * *} \\
(0.088)\end{array}$ & $\begin{array}{c}-0.933^{* * *} \\
(0.109)\end{array}$ & $\begin{array}{c}-0.699 * * * \\
(0.108)\end{array}$ & $\begin{array}{c}0.403^{* * *} \\
(0.098)\end{array}$ \\
\hline lag 3 & $\begin{array}{c}0.661^{* * *} \\
(0.068)\end{array}$ & $\begin{array}{c}0.645^{* * *} \\
(0.068)\end{array}$ & $\begin{array}{c}0.370^{* * *} \\
(0.089)\end{array}$ & $\begin{array}{c}-0.257^{* * * *} \\
(0.077)\end{array}$ & $\begin{array}{c}-0.853^{* * *} \\
(0.103)\end{array}$ & $\begin{array}{c}-0.516^{* * *} \\
(0.110)\end{array}$ & $\begin{array}{l}-0.356 \\
(0.343)\end{array}$ \\
\hline $\operatorname{lag} 4$ & $\begin{array}{l}-0.077 \\
(0.050)\end{array}$ & $\begin{array}{l}-0.109 \\
(0.069)\end{array}$ & $\begin{array}{c}-0.658^{* * * *} \\
(0.090)\end{array}$ & $\begin{array}{c}-0.404^{* * * *} \\
(0.101)\end{array}$ & $\begin{array}{c}0.460 * * * \\
(0.112)\end{array}$ & $\begin{array}{c}1.639^{* * *} \\
(0.134)\end{array}$ & $\begin{array}{c}-1.352^{* *} \\
(0.590)\end{array}$ \\
\hline $\operatorname{lag} 5$ & $\begin{array}{c}0.319^{* * * *} \\
(0.038)\end{array}$ & $\begin{array}{l}0.109^{*} \\
(0.059)\end{array}$ & $\begin{array}{c}0.238^{* * * *} \\
(0.070)\end{array}$ & $\begin{array}{l}-0.079 \\
(0.081)\end{array}$ & $\begin{array}{l}-0.106 \\
(0.104)\end{array}$ & $\begin{array}{c}-3.284^{* * *} \\
(0.463)\end{array}$ & $\begin{array}{c}-3.027^{* * *} \\
(0.562)\end{array}$ \\
\hline $\begin{array}{l}\text { Fixed effects } \\
\text { Item }\end{array}$ & Yes & Yes & Yes & Yes & Yes & Yes & Yes \\
\hline $\begin{array}{l}R \text {-squared } \\
\text { Observations }\end{array}$ & $\begin{array}{c}0.066 \\
45,507\end{array}$ & $\begin{array}{c}0.143 \\
42,473\end{array}$ & $\begin{array}{c}0.196 \\
39,439\end{array}$ & $\begin{array}{c}0.247 \\
36,405\end{array}$ & $\begin{array}{c}0.268 \\
33,371\end{array}$ & $\begin{array}{c}0.291 \\
30,337\end{array}$ & $\begin{array}{c}0.318 \\
27,303\end{array}$ \\
\hline
\end{tabular}

Note: The table presents the full estimation results of model (??) using the sales-weighted exchange rate, $e r^{*}$, for $h=0,2, \cdots, 10,12$ and $L=5$. Robust standard errors (clustered by product) are in parentheses. The notation ${ }^{* * *}$ indicates significance at $1 \%$ level, ${ }^{* *}$ at $5 \%$ level, ${ }^{*}$ at $10 \%$ level. 
Table A8: ERPT and the retailer size: imported products with $L=5$

\begin{tabular}{|c|c|c|c|c|c|c|c|}
\hline \multirow[b]{2}{*}{ Variable } & \multicolumn{7}{|c|}{ Horizon (h) } \\
\hline & 0 & 2 & 4 & 6 & 8 & 10 & 12 \\
\hline$\Delta \ln e r$ & $\begin{array}{c}-0.008 \\
(0.006)\end{array}$ & $\begin{array}{c}0.035^{* * *} \\
(0.011)\end{array}$ & $\begin{array}{c}0.191^{* * *} \\
(0.019)\end{array}$ & $\begin{array}{c}0.281^{* * *} \\
(0.020)\end{array}$ & $\begin{array}{c}0.404^{* * *} \\
(0.023)\end{array}$ & $\begin{array}{c}0.422^{* * *} \\
(0.022)\end{array}$ & $\begin{array}{c}0.509^{* * *} \\
(0.030)\end{array}$ \\
\hline $\operatorname{lag} 1$ & $\begin{array}{c}0.034^{* * *} \\
(0.009)\end{array}$ & $\begin{array}{c}0.149^{* * *} \\
(0.011)\end{array}$ & $\begin{array}{c}0.200^{* * *} \\
(0.013)\end{array}$ & $\begin{array}{c}0.278^{* * *} \\
(0.014)\end{array}$ & $\begin{array}{c}0.275^{* * *} \\
(0.015)\end{array}$ & $\begin{array}{c}0.288^{* * *} \\
(0.018)\end{array}$ & $\begin{array}{c}0.165^{* * *} \\
(0.012)\end{array}$ \\
\hline $\operatorname{lag} 2$ & $\begin{array}{c}0.104^{* * *} \\
(0.010)\end{array}$ & $\begin{array}{c}0.223^{* * *} \\
(0.012)\end{array}$ & $\begin{array}{c}0.246^{* * *} \\
(0.011)\end{array}$ & $\begin{array}{c}0.289^{* * *} \\
(0.013)\end{array}$ & $\begin{array}{c}0.292^{* * *} \\
(0.014)\end{array}$ & $\begin{array}{c}0.236^{* * *} \\
(0.016)\end{array}$ & $\begin{array}{c}0.050^{* *} \\
(0.020)\end{array}$ \\
\hline $\operatorname{lag} 3$ & $\begin{array}{c}0.052^{* * *} \\
(0.008)\end{array}$ & $\begin{array}{c}0.109 * * * \\
(0.010)\end{array}$ & $\begin{array}{c}0.202^{* * *} \\
(0.013)\end{array}$ & $\begin{array}{c}0.235^{* * *} \\
(0.012)\end{array}$ & $\begin{array}{c}0.298^{* * *} \\
(0.016)\end{array}$ & $\begin{array}{c}0.285^{* * *} \\
(0.014)\end{array}$ & $\begin{array}{c}0.202^{* * *} \\
(0.014)\end{array}$ \\
\hline $\operatorname{lag} 4$ & $\begin{array}{c}0.054^{* * *} \\
(0.008)\end{array}$ & $\begin{array}{c}0.095^{* * *} \\
(0.010)\end{array}$ & $\begin{array}{c}0.140^{* * *} \\
(0.009)\end{array}$ & $\begin{array}{c}0.219^{* * *} \\
(0.011)\end{array}$ & $\begin{array}{c}0.212^{* * *} \\
(0.013)\end{array}$ & $\begin{array}{c}0.220^{* * *} \\
(0.014)\end{array}$ & $\begin{array}{c}0.198^{* * *} \\
(0.032)\end{array}$ \\
\hline $\operatorname{lag} 5$ & $\begin{array}{c}0.058^{* * *} \\
(0.008)\end{array}$ & $\begin{array}{c}0.152^{* * * *} \\
(0.013)\end{array}$ & $\begin{array}{c}0.190^{* * *} \\
(0.013)\end{array}$ & $\begin{array}{c}0.223^{* * *} \\
(0.015)\end{array}$ & $\begin{array}{c}0.202^{* * *} \\
(0.016)\end{array}$ & $\begin{array}{c}0.060^{* * *} \\
(0.013)\end{array}$ & $\begin{array}{c}-0.460^{* * *} \\
(0.056)\end{array}$ \\
\hline$\Delta \ln e r \times$ Large & $\begin{array}{c}0.014 \\
(0.009)\end{array}$ & $\begin{array}{c}0.044^{* *} \\
(0.018)\end{array}$ & $\begin{array}{c}0.127^{* * * *} \\
(0.022)\end{array}$ & $\begin{array}{c}0.122^{* * *} \\
(0.027)\end{array}$ & $\begin{array}{c}0.113^{* * *} \\
(0.030)\end{array}$ & $\begin{array}{c}0.131^{* * * *} \\
(0.028)\end{array}$ & $\begin{array}{c}0.096^{* * *} \\
(0.029)\end{array}$ \\
\hline \multicolumn{8}{|l|}{$\Delta \ln p$} \\
\hline $\operatorname{lag} 1$ & $\begin{array}{c}-0.214^{* * *} \\
(0.017)\end{array}$ & $\begin{array}{c}-0.350^{* * *} \\
(0.018)\end{array}$ & $\begin{array}{c}-0.424^{* * *} \\
(0.020)\end{array}$ & $\begin{array}{c}-0.596^{* * *} \\
(0.021)\end{array}$ & $\begin{array}{c}-0.718^{* * *} \\
(0.020)\end{array}$ & $\begin{array}{c}-0.802^{* * *} \\
(0.023)\end{array}$ & $\begin{array}{c}-0.824^{* * *} \\
(0.024)\end{array}$ \\
\hline $\operatorname{lag} 2$ & $\begin{array}{c}-0.129^{* * *} \\
(0.014)\end{array}$ & $\begin{array}{c}-0.215^{* * *} \\
(0.016)\end{array}$ & $\begin{array}{c}-0.345^{* * *} \\
(0.017)\end{array}$ & $\begin{array}{c}-0.546^{* * *} \\
(0.019)\end{array}$ & $\begin{array}{c}-0.656^{* * *} \\
(0.019)\end{array}$ & $\begin{array}{c}-0.751^{* * *} \\
(0.025)\end{array}$ & $\begin{array}{c}-0.768^{* * *} \\
(0.026)\end{array}$ \\
\hline $\operatorname{lag} 3$ & $\begin{array}{c}-0.060^{* * *} \\
(0.011)\end{array}$ & $\begin{array}{c}-0.139^{* * *} \\
(0.016)\end{array}$ & $\begin{array}{c}-0.311^{* * *} \\
(0.019)\end{array}$ & $\begin{array}{c}-0.481^{* * *} \\
(0.018)\end{array}$ & $\begin{array}{c}-0.612^{* * *} \\
(0.020)\end{array}$ & $\begin{array}{c}-0.709^{* * *} \\
(0.023)\end{array}$ & $\begin{array}{c}-0.761^{* * *} \\
(0.031)\end{array}$ \\
\hline $\operatorname{lag} 4$ & $\begin{array}{c}-0.027^{* *} \\
(0.011)\end{array}$ & $\begin{array}{c}-0.122^{* * *} \\
(0.015)\end{array}$ & $\begin{array}{c}-0.288^{* * *} \\
(0.018)\end{array}$ & $\begin{array}{c}-0.410^{* * *} \\
(0.018)\end{array}$ & $\begin{array}{c}-0.546^{* * *} \\
(0.019)\end{array}$ & $\begin{array}{c}-0.667^{* * *} \\
(0.023)\end{array}$ & $\begin{array}{c}-0.771^{* * *} \\
(0.034)\end{array}$ \\
\hline $\operatorname{lag} 5$ & $\begin{array}{l}-0.013 \\
(0.010)\end{array}$ & $\begin{array}{c}-0.123^{* * *} \\
(0.014)\end{array}$ & $\begin{array}{c}-0.243^{* * *} \\
(0.016)\end{array}$ & $\begin{array}{c}-0.358^{* * *} \\
(0.017)\end{array}$ & $\begin{array}{c}-0.464^{* * *} \\
(0.018)\end{array}$ & $\begin{array}{c}-0.558^{* * *} \\
(0.024)\end{array}$ & $\begin{array}{c}-0.656^{* * *} \\
(0.037)\end{array}$ \\
\hline \multicolumn{8}{|l|}{$\Delta \ln q$} \\
\hline $\operatorname{lag} 1$ & & $\begin{array}{c}-0.002 \\
(0.002)\end{array}$ & $\begin{array}{c}-0.004^{* *} \\
(0.002)\end{array}$ & $\begin{array}{l}-0.004 \\
(0.002)\end{array}$ & $\begin{array}{l}-0.003 \\
(0.002)\end{array}$ & $\begin{array}{l}-0.000 \\
(0.002)\end{array}$ & $\begin{array}{l}0.003^{*} \\
(0.002)\end{array}$ \\
\hline $\operatorname{lag} 2$ & $\begin{array}{c}-0.002^{*} \\
(0.001)\end{array}$ & $\begin{array}{l}-0.002 \\
(0.002)\end{array}$ & $\begin{array}{l}-0.003 \\
(0.002)\end{array}$ & $\begin{array}{l}-0.002 \\
(0.002)\end{array}$ & $\begin{array}{l}-0.003 \\
(0.002)\end{array}$ & $\begin{array}{c}0.001 \\
(0.002)\end{array}$ & $\begin{array}{c}0.004 \\
(0.002)\end{array}$ \\
\hline $\operatorname{lag} 3$ & $\begin{array}{l}-0.000 \\
(0.001)\end{array}$ & $\begin{array}{l}-0.002 \\
(0.002)\end{array}$ & $\begin{array}{l}-0.001 \\
(0.002)\end{array}$ & $\begin{array}{l}-0.001 \\
(0.002)\end{array}$ & $\begin{array}{c}0.001 \\
(0.002)\end{array}$ & $\begin{array}{c}0.004 \\
(0.002)\end{array}$ & $\begin{array}{c}0.003 \\
(0.002)\end{array}$ \\
\hline $\operatorname{lag} 4$ & $\begin{array}{l}-0.000 \\
(0.001)\end{array}$ & $\begin{array}{l}-0.001 \\
(0.002)\end{array}$ & $\begin{array}{l}-0.000 \\
(0.002)\end{array}$ & $\begin{array}{l}-0.002 \\
(0.002)\end{array}$ & $\begin{array}{c}0.002 \\
(0.002)\end{array}$ & $\begin{array}{c}0.002 \\
(0.003)\end{array}$ & $\begin{array}{c}0.008^{* * *} \\
(0.002)\end{array}$ \\
\hline $\operatorname{lag} 5$ & $\begin{array}{l}-0.001 \\
(0.001)\end{array}$ & $\begin{array}{l}-0.001 \\
(0.001)\end{array}$ & $\begin{array}{l}-0.002 \\
(0.002)\end{array}$ & $\begin{array}{c}0.000 \\
(0.002)\end{array}$ & $\begin{array}{c}0.001 \\
(0.002)\end{array}$ & $\begin{array}{l}-0.000 \\
(0.002)\end{array}$ & $\begin{array}{c}0.003 \\
(0.002)\end{array}$ \\
\hline \multicolumn{8}{|l|}{$\Delta \ln C P I$} \\
\hline $\operatorname{lag} 1$ & $\begin{array}{c}0.807^{* * *} \\
(0.090)\end{array}$ & $\begin{array}{c}1.012^{* * *} \\
(0.155)\end{array}$ & $\begin{array}{c}1.444^{* * *} \\
(0.168)\end{array}$ & $\begin{array}{c}1.872^{* * *} \\
(0.201)\end{array}$ & $\begin{array}{c}2.780^{* * *} \\
(0.255)\end{array}$ & $\begin{array}{c}3.645^{* * *} \\
(0.379)\end{array}$ & $\begin{array}{c}2.294^{* * *} \\
(0.218)\end{array}$ \\
\hline $\operatorname{lag} 2$ & $\begin{array}{l}-0.120 \\
(0.092)\end{array}$ & $\begin{array}{l}-0.138 \\
(0.121)\end{array}$ & $\begin{array}{c}-0.501^{* * *} \\
(0.120)\end{array}$ & $\begin{array}{c}-0.323^{* * *} \\
(0.106)\end{array}$ & $\begin{array}{l}-0.060 \\
(0.168)\end{array}$ & $\begin{array}{l}-0.156 \\
(0.140)\end{array}$ & $\begin{array}{c}-0.987^{* * *} \\
(0.277)\end{array}$ \\
\hline $\operatorname{lag} 3$ & $\begin{array}{l}-0.029 \\
(0.103)\end{array}$ & $\begin{array}{l}-0.021 \\
(0.119)\end{array}$ & $\begin{array}{c}-0.402^{* * *} \\
(0.142)\end{array}$ & $\begin{array}{c}-0.366^{* * *} \\
(0.138)\end{array}$ & $\begin{array}{l}-0.153 \\
(0.107)\end{array}$ & $\begin{array}{l}0.119 \\
(0.117)\end{array}$ & $\begin{array}{c}-0.663^{* * * *} \\
(0.131)\end{array}$ \\
\hline $\operatorname{lag} 4$ & $\begin{array}{c}0.109 \\
(0.091)\end{array}$ & $\begin{array}{c}-0.537^{* * *} \\
(0.123)\end{array}$ & $\begin{array}{c}-0.736^{* * *} \\
(0.145)\end{array}$ & $\begin{array}{c}-0.789^{* * *} \\
(0.166)\end{array}$ & $\begin{array}{c}-0.949^{* * *} \\
(0.168)\end{array}$ & $\begin{array}{c}-1.227^{* * *} \\
(0.171)\end{array}$ & $\begin{array}{c}-2.753^{* * *} \\
(0.362)\end{array}$ \\
\hline $\operatorname{lag} 5$ & $\begin{array}{c}-0.802^{* * *} \\
(0.085)\end{array}$ & $\begin{array}{c}-1.344^{* * *} \\
(0.131)\end{array}$ & $\begin{array}{c}-1.022^{* * *} \\
(0.164)\end{array}$ & $\begin{array}{l}-0.246 \\
(0.212)\end{array}$ & $\begin{array}{c}0.957^{* * *} \\
(0.220)\end{array}$ & $\begin{array}{c}1.220^{* * *} \\
(0.240)\end{array}$ & $\begin{array}{c}0.595^{* * *} \\
(0.189)\end{array}$ \\
\hline $\begin{array}{l}\text { Fixed effects } \\
\text { Item }\end{array}$ & Yes & Yes & Yes & Yes & Yes & Yes & Yes \\
\hline$R$-squared & $\begin{array}{c}0.092 \\
25.740\end{array}$ & $\begin{array}{c}0.216 \\
24.024\end{array}$ & $\begin{array}{c}0.338 \\
22.308\end{array}$ & $\begin{array}{c}0.436 \\
20.592\end{array}$ & 0.491 & 0.510 & 0.529 \\
\hline
\end{tabular}

Note: The table presents the full estimation results of model (4) for $h=0,2, \cdots, 10,12$ and $L=5$. Robust standard errors (clustered by product) are in parentheses. The notation *** indicates significance at $1 \%$ level, ${ }^{* *}$ at $5 \%$ level, $*$ at $10 \%$ level. 
Table A9: ERPT and the retailer size: domestic products with $L=5$

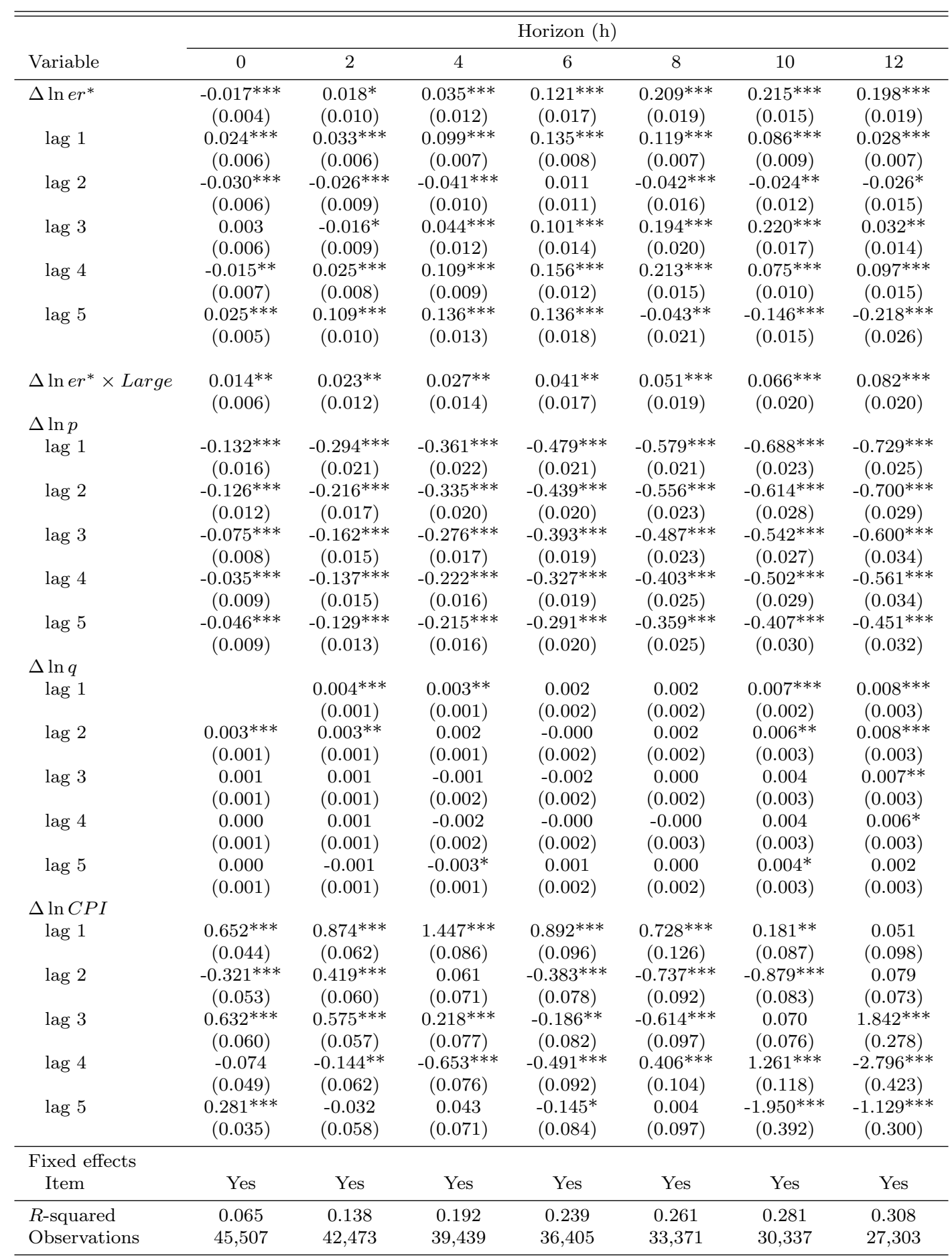

Note: The table presents the full estimation results of model (4) using the sales-weighted exchange rate, $e r^{*}$, for $h=0,2, \cdots, 10,12$ and $L=5$. Robust standard errors (clustered by product) are in parentheses. The notation $* * *$ indicates significance at $1 \%$ level, $* *$ at $5 \%$ level, $*$ at $10 \%$ level. 
Table A10: Asymmetric ERPT and the retailer size: imported products with $L=5$

\begin{tabular}{|c|c|c|c|c|c|c|c|}
\hline \multirow[b]{2}{*}{ Variable } & \multicolumn{7}{|c|}{ Horizon (h) } \\
\hline & 0 & 2 & 4 & 6 & 8 & 10 & 12 \\
\hline$\Delta \ln e r$ & $\begin{array}{c}-0.026^{* *} \\
(0.012)\end{array}$ & $\begin{array}{c}-0.071^{* * *} \\
(0.023)\end{array}$ & $\begin{array}{c}0.101^{* * *} \\
(0.039)\end{array}$ & $\begin{array}{c}0.172^{* * *} \\
(0.041)\end{array}$ & $\begin{array}{c}0.152^{* * *} \\
(0.043)\end{array}$ & $\begin{array}{l}0.094^{* *} \\
(0.045)\end{array}$ & $\begin{array}{c}0.113^{* * * *} \\
(0.040)\end{array}$ \\
\hline $\operatorname{lag} 1$ & $\begin{array}{c}0.037^{* * *} \\
(0.010)\end{array}$ & $\begin{array}{c}0.166^{* * *} \\
(0.014)\end{array}$ & $\begin{array}{c}0.221^{* * *} \\
(0.016)\end{array}$ & $\begin{array}{c}0.299 * * * \\
(0.018)\end{array}$ & $\begin{array}{c}0.321^{* * *} \\
(0.020)\end{array}$ & $\begin{array}{c}0.349^{* * *} \\
(0.024)\end{array}$ & $\begin{array}{c}0.260^{* * * *} \\
(0.022)\end{array}$ \\
\hline $\operatorname{lag} 2$ & $\begin{array}{c}0.104^{* * *} \\
(0.010)\end{array}$ & $\begin{array}{c}0.223^{* * *} \\
(0.012)\end{array}$ & $\begin{array}{c}0.247^{* * *} \\
(0.012)\end{array}$ & $\begin{array}{c}0.294^{* * *} \\
(0.014)\end{array}$ & $\begin{array}{c}0.299^{* * *} \\
(0.015)\end{array}$ & $\begin{array}{c}0.244^{* * *} \\
(0.017)\end{array}$ & $\begin{array}{c}0.075^{* * *} \\
(0.018)\end{array}$ \\
\hline $\operatorname{lag} 3$ & $\begin{array}{c}0.056^{* * *} \\
(0.009)\end{array}$ & $\begin{array}{c}0.137 * * * \\
(0.013)\end{array}$ & $\begin{array}{c}0.227^{* * *} \\
(0.018)\end{array}$ & $\begin{array}{c}0.266^{* * *} \\
(0.016)\end{array}$ & $\begin{array}{c}0.370^{* * * *} \\
(0.021)\end{array}$ & $\begin{array}{c}0.378^{* * *} \\
(0.021)\end{array}$ & $\begin{array}{c}0.305^{* * *} \\
(0.022)\end{array}$ \\
\hline $\operatorname{lag} 4$ & $\begin{array}{c}0.055^{* * *} \\
(0.008)\end{array}$ & $\begin{array}{c}0.104^{* * *} \\
(0.010)\end{array}$ & $\begin{array}{c}0.151^{* * *} \\
(0.011)\end{array}$ & $\begin{array}{c}0.230^{* * *} \\
(0.012)\end{array}$ & $\begin{array}{c}0.236^{* * *} \\
(0.015)\end{array}$ & $\begin{array}{c}0.255^{* * *} \\
(0.019)\end{array}$ & $\begin{array}{c}0.296^{* * *} \\
(0.046)\end{array}$ \\
\hline $\operatorname{lag} 5$ & $\begin{array}{c}0.057^{* * *} \\
(0.009)\end{array}$ & $\begin{array}{c}0.150^{* * *} \\
(0.013)\end{array}$ & $\begin{array}{c}0.190^{* * *} \\
(0.013)\end{array}$ & $\begin{array}{c}0.229^{* * *} * \\
(0.015)\end{array}$ & $\begin{array}{c}0.218^{* * *} \\
(0.016)\end{array}$ & $\begin{array}{c}0.073^{* * *} \\
(0.014)\end{array}$ & $\begin{array}{c}-0.423^{* * *} \\
(0.047)\end{array}$ \\
\hline$\Delta \ln e r \times d$ & $\begin{array}{c}0.030 \\
(0.019)\end{array}$ & $\begin{array}{c}0.179 * * * \\
(0.042)\end{array}$ & $\begin{array}{c}0.150^{* *} \\
(0.063)\end{array}$ & $\begin{array}{c}0.183^{* * *} \\
(0.066)\end{array}$ & $\begin{array}{c}0.417 * * * \\
(0.069)\end{array}$ & $\begin{array}{c}0.535^{* * *} \\
(0.073)\end{array}$ & $\begin{array}{c}0.629 * * * \\
(0.072)\end{array}$ \\
\hline$\Delta \ln e r \times$ Large & $\begin{array}{c}0.024 \\
(0.015)\end{array}$ & $\begin{array}{c}0.004 \\
(0.025)\end{array}$ & $\begin{array}{c}0.034 \\
(0.040)\end{array}$ & $\begin{array}{c}0.044 \\
(0.049)\end{array}$ & $\begin{array}{c}0.044 \\
(0.055)\end{array}$ & $\begin{array}{c}0.075 \\
(0.050)\end{array}$ & $\begin{array}{c}0.048 \\
(0.045)\end{array}$ \\
\hline$\Delta \ln e r \times d \times$ Large & $\begin{array}{l}-0.017 \\
(0.027)\end{array}$ & $\begin{array}{c}0.071 \\
(0.050)\end{array}$ & $\begin{array}{c}0.167^{* *} \\
(0.068)\end{array}$ & $\begin{array}{l}0.138^{*} \\
(0.076)\end{array}$ & $\begin{array}{c}0.121 \\
(0.080)\end{array}$ & $\begin{array}{l}0.096 \\
(0.069)\end{array}$ & $\begin{array}{c}0.079 \\
(0.065)\end{array}$ \\
\hline $\begin{array}{l}\Delta \ln p \\
\quad \operatorname{lag} 1\end{array}$ & $\begin{array}{c}-0.213^{* * *} \\
(0.017)\end{array}$ & $\begin{array}{c}-0.345^{* * *} \\
(0.018)\end{array}$ & $\begin{array}{c}-0.418^{* * *} \\
(0.020)\end{array}$ & $\begin{array}{c}-0.589^{* * *} \\
(0.021)\end{array}$ & $\begin{array}{c}-0.710^{* * *} \\
(0.019)\end{array}$ & $\begin{array}{c}-0.795^{* * *} \\
(0.023)\end{array}$ & $\begin{array}{c}-0.806^{* * *} \\
(0.024)\end{array}$ \\
\hline lag 2 & $\begin{array}{c}-0.128 * * * \\
(0.014)\end{array}$ & $\begin{array}{c}-0.205^{* * *} \\
(0.016)\end{array}$ & $\begin{array}{c}-0.334^{* * *} \\
(0.017)\end{array}$ & $\begin{array}{c}-0.533^{* * *} \\
(0.019)\end{array}$ & $\begin{array}{c}-0.638^{* * *} \\
(0.019)\end{array}$ & $\begin{array}{c}-0.731^{* * *} \\
(0.025)\end{array}$ & $\begin{array}{c}-0.738^{* * *} * \\
(0.026)\end{array}$ \\
\hline $\operatorname{lag} 3$ & $\begin{array}{c}-0.059^{* * *} \\
(0.011)\end{array}$ & $\begin{array}{c}-0.126^{* * *} \\
(0.016)\end{array}$ & $\begin{array}{c}-0.297 * * * \\
(0.019)\end{array}$ & $\begin{array}{c}-0.466^{* * *} \\
(0.019)\end{array}$ & $\begin{array}{c}-0.589^{* * *} \\
(0.020)\end{array}$ & $\begin{array}{c}-0.683^{* * *} \\
(0.023)\end{array}$ & $\begin{array}{c}-0.724^{* * *} \\
(0.030)\end{array}$ \\
\hline $\operatorname{lag} 4$ & $\begin{array}{c}-0.025^{* *} \\
(0.011)\end{array}$ & $\begin{array}{c}-0.106^{* * * *} \\
(0.015)\end{array}$ & $\begin{array}{c}-0.271^{* * *} \\
(0.018)\end{array}$ & $\begin{array}{c}-0.391 * * * \\
(0.018)\end{array}$ & $\begin{array}{c}-0.517^{* * * *} \\
(0.018)\end{array}$ & $\begin{array}{c}-0.633^{* * *} \\
(0.022)\end{array}$ & $\begin{array}{c}-0.726^{* * *} \\
(0.034)\end{array}$ \\
\hline $\operatorname{lag} 5$ & $\begin{array}{c}-0.011 \\
(0.010)\end{array}$ & $\begin{array}{c}-0.107^{* * *} \\
(0.014)\end{array}$ & $\begin{array}{c}-0.226^{* * *} \\
(0.016)\end{array}$ & $\begin{array}{c}-0.339^{* * *} \\
(0.017)\end{array}$ & $\begin{array}{c}-0.434^{* * *} \\
(0.018)\end{array}$ & $\begin{array}{c}-0.522^{* * *} \\
(0.024)\end{array}$ & $\begin{array}{c}-0.611^{* * *} * \\
(0.037)\end{array}$ \\
\hline $\begin{array}{l}\Delta \ln q \\
\quad \operatorname{lag} 1\end{array}$ & & $\begin{array}{l}-0.002 \\
(0.002)\end{array}$ & $\begin{array}{c}-0.004^{*} \\
(0.002)\end{array}$ & $\begin{array}{l}-0.003 \\
(0.002)\end{array}$ & $\begin{array}{l}-0.003 \\
(0.002)\end{array}$ & $\begin{array}{c}0.000 \\
(0.002)\end{array}$ & $\begin{array}{l}0.004^{*} \\
(0.002)\end{array}$ \\
\hline $\operatorname{lag} 2$ & $\begin{array}{c}-0.002 \\
(0.001)\end{array}$ & $\begin{array}{l}-0.001 \\
(0.002)\end{array}$ & $\begin{array}{l}-0.002 \\
(0.002)\end{array}$ & $\begin{array}{l}-0.001 \\
(0.002)\end{array}$ & $\begin{array}{l}-0.001 \\
(0.002)\end{array}$ & $\begin{array}{c}0.003 \\
(0.002)\end{array}$ & $\begin{array}{c}0.006^{* *} \\
(0.002)\end{array}$ \\
\hline $\operatorname{lag} 3$ & $\begin{array}{l}-0.000 \\
(0.001)\end{array}$ & $\begin{array}{c}-0.001 \\
(0.002)\end{array}$ & $\begin{array}{l}-0.001 \\
(0.002)\end{array}$ & $\begin{array}{l}-0.001 \\
(0.002)\end{array}$ & $\begin{array}{c}0.002 \\
(0.002)\end{array}$ & $\begin{array}{c}0.005^{* * *} \\
(0.003)\end{array}$ & $\begin{array}{c}0.005^{* *} \\
(0.002)\end{array}$ \\
\hline $\operatorname{lag} 4$ & $\begin{array}{c}0.000 \\
(0.001)\end{array}$ & $\begin{array}{c}-0.000 \\
(0.002)\end{array}$ & $\begin{array}{c}0.000 \\
(0.002)\end{array}$ & $\begin{array}{l}-0.001 \\
(0.002)\end{array}$ & $\begin{array}{c}0.003 \\
(0.002)\end{array}$ & $\begin{array}{c}0.004 \\
(0.003)\end{array}$ & $\begin{array}{c}0.011^{* * *} \\
(0.002)\end{array}$ \\
\hline $\operatorname{lag} 5$ & $\begin{array}{l}-0.001 \\
(0.001)\end{array}$ & $\begin{array}{c}0.000 \\
(0.001)\end{array}$ & $\begin{array}{l}-0.001 \\
(0.002)\end{array}$ & $\begin{array}{c}0.001 \\
(0.002)\end{array}$ & $\begin{array}{c}0.003 \\
(0.002)\end{array}$ & $\begin{array}{c}0.002 \\
(0.002)\end{array}$ & $\begin{array}{c}0.006^{* * *} \\
(0.002)\end{array}$ \\
\hline $\begin{array}{c}\Delta \ln C P I \\
\operatorname{lag} 1\end{array}$ & $\begin{array}{c}0.805^{* * *} \\
(0.090)\end{array}$ & $\begin{array}{c}1.027^{* * *} \\
(0.158)\end{array}$ & $\begin{array}{c}1.536^{* * *} \\
(0.194)\end{array}$ & $\begin{array}{c}2.008^{* * *} \\
(0.233)\end{array}$ & $\begin{array}{c}3.121^{* * *} \\
(0.306)\end{array}$ & $\begin{array}{c}4.102^{* * *} \\
(0.440)\end{array}$ & $\begin{array}{c}3.163^{* * *} \\
(0.346)\end{array}$ \\
\hline lag 2 & $\begin{array}{c}-0.093 \\
(0.093)\end{array}$ & $\begin{array}{c}0.063 \\
(0.131)\end{array}$ & $\begin{array}{c}-0.284^{*} \\
(0.152)\end{array}$ & $\begin{array}{l}-0.048 \\
(0.128)\end{array}$ & $\begin{array}{c}0.583^{* * *} \\
(0.194)\end{array}$ & $\begin{array}{c}0.729 * * * \\
(0.171)\end{array}$ & $\begin{array}{c}0.116 \\
(0.207)\end{array}$ \\
\hline lag 3 & $\begin{array}{c}-0.048 \\
(0.104)\end{array}$ & $\begin{array}{l}-0.109 \\
(0.130)\end{array}$ & $\begin{array}{c}-0.511 * * * \\
(0.153)\end{array}$ & $\begin{array}{c}-0.480^{* * *} \\
(0.156)\end{array}$ & $\begin{array}{c}-0.346^{* * *} \\
(0.119)\end{array}$ & $\begin{array}{c}-0.174 \\
(0.144)\end{array}$ & $\begin{array}{c}-0.872^{* * *} \\
(0.134)\end{array}$ \\
\hline lag 4 & $\begin{array}{c}0.127 \\
(0.090)\end{array}$ & $\begin{array}{c}-0.403^{* * *} \\
(0.121)\end{array}$ & $\begin{array}{c}-0.577 * * * \\
(0.142)\end{array}$ & $\begin{array}{c}-0.565^{* * *} \\
(0.169)\end{array}$ & $\begin{array}{c}-0.480^{* * *} \\
(0.166)\end{array}$ & $\begin{array}{c}-0.595^{* * *} \\
(0.160)\end{array}$ & $\begin{array}{c}-1.807^{* * *} * \\
(0.287)\end{array}$ \\
\hline $\operatorname{lag} 5$ & $\begin{array}{c}-0.820^{* * *} \\
(0.089)\end{array}$ & $\begin{array}{c}-1.457^{* * * *} \\
(0.138)\end{array}$ & $\begin{array}{c}-1.076^{* * *} \\
(0.165)\end{array}$ & $\begin{array}{c}-0.302 \\
(0.208)\end{array}$ & $\begin{array}{c}0.853^{* * *} \\
(0.216)\end{array}$ & $\begin{array}{c}1.057^{* * *} \\
(0.226)\end{array}$ & $\begin{array}{l}0.350^{*} \\
(0.205)\end{array}$ \\
\hline $\begin{array}{l}\text { Fixed effects } \\
\text { Item }\end{array}$ & Yes & Yes & Yes & Yes & Yes & Yes & Yes \\
\hline $\begin{array}{l}R \text {-squared } \\
\text { Observations }\end{array}$ & $\begin{array}{c}0.092 \\
25,740\end{array}$ & $\begin{array}{c}0.220 \\
24,024\end{array}$ & $\begin{array}{c}0.340 \\
22,308\end{array}$ & $\begin{array}{c}0.438 \\
20,592\end{array}$ & $\begin{array}{c}0.497 \\
18,876\end{array}$ & $\begin{array}{c}0.520 \\
17,160\end{array}$ & $\begin{array}{c}0.542 \\
15,444\end{array}$ \\
\hline
\end{tabular}

Note: $\overline{\text { The table presents the full estimation results of model (5) for } h=0,2, \cdots, 10,12 \text { and } L=5 \text {. Robust }}$ standard errors (clustered by product) are in parentheses. The notation $* * *$ indicates significance at $1 \%$ level, ${ }^{* *}$ at $5 \%$ level, ${ }^{*}$ at $10 \%$ level. 
Table A11: Asymmetric ERPT and the retailer size: domestic products with $L=5$

\begin{tabular}{|c|c|c|c|c|c|c|c|}
\hline \multirow[b]{2}{*}{ Variable } & \multicolumn{7}{|c|}{ Horizon (h) } \\
\hline & 0 & 2 & 4 & 6 & 8 & 10 & 12 \\
\hline$\Delta \ln e r^{*}$ & $\begin{array}{c}-0.027^{* * *} \\
(0.008)\end{array}$ & $\begin{array}{c}-0.052^{* * *} \\
(0.018)\end{array}$ & $\begin{array}{c}-0.029 \\
(0.024)\end{array}$ & $\begin{array}{c}0.079 * * * \\
(0.028)\end{array}$ & $\begin{array}{c}0.269 * * * \\
(0.033)\end{array}$ & $\begin{array}{c}0.317^{* * *} \\
(0.034)\end{array}$ & $\begin{array}{c}0.335^{* * *} \\
(0.030)\end{array}$ \\
\hline $\operatorname{lag} 1$ & $\begin{array}{c}0.025^{* * *} \\
(0.006)\end{array}$ & $\begin{array}{c}0.040^{* * *} \\
(0.007)\end{array}$ & $\begin{array}{c}0.106^{* * *} \\
(0.007)\end{array}$ & $\begin{array}{c}0.138^{* * *} \\
(0.009)\end{array}$ & $\begin{array}{c}0.110^{* * *} \\
(0.009)\end{array}$ & $\begin{array}{c}0.079^{* * *} \\
(0.010)\end{array}$ & $\begin{array}{c}0.023^{* * *} \\
(0.007)\end{array}$ \\
\hline lag 2 & $\begin{array}{c}-0.030^{* * *} \\
(0.006)\end{array}$ & $\begin{array}{c}-0.030^{* * * *} \\
(0.010)\end{array}$ & $\begin{array}{c}-0.047^{* * *} * \\
(0.011)\end{array}$ & $\begin{array}{c}0.006 \\
(0.013)\end{array}$ & $\begin{array}{l}-0.024 \\
(0.021)\end{array}$ & $\begin{array}{c}0.032 \\
(0.023)\end{array}$ & $\begin{array}{c}0.033 \\
(0.024)\end{array}$ \\
\hline $\operatorname{lag} 3$ & $\begin{array}{c}0.005 \\
(0.007)\end{array}$ & $\begin{array}{c}-0.001 \\
(0.009)\end{array}$ & $\begin{array}{c}0.055^{* * *} \\
(0.013)\end{array}$ & $\begin{array}{c}0.109^{* * *} \\
(0.017)\end{array}$ & $\begin{array}{c}0.170^{* * *} \\
(0.025)\end{array}$ & $\begin{array}{c}0.161^{* * *} \\
(0.025)\end{array}$ & $\begin{array}{c}-0.028 \\
(0.021)\end{array}$ \\
\hline $\operatorname{lag} 4$ & $\begin{array}{c}-0.014^{* *} \\
(0.007)\end{array}$ & $\begin{array}{c}0.026^{* * *} \\
(0.008)\end{array}$ & $\begin{array}{c}0.113^{* * *} \\
(0.009)\end{array}$ & $\begin{array}{c}0.157^{* * *} \\
(0.012)\end{array}$ & $\begin{array}{c}0.210^{* * *} \\
(0.015)\end{array}$ & $\begin{array}{c}0.066^{* * *} \\
(0.010)\end{array}$ & $\begin{array}{c}0.056^{* * *} \\
(0.018)\end{array}$ \\
\hline $\operatorname{lag} 5$ & $\begin{array}{c}0.024^{* * *} \\
(0.005)\end{array}$ & $\begin{array}{c}0.101^{* * *} \\
(0.010)\end{array}$ & $\begin{array}{c}0.126^{* * *} \\
(0.015)\end{array}$ & $\begin{array}{c}0.129^{* * *} \\
(0.020)\end{array}$ & $\begin{array}{l}-0.024 \\
(0.024)\end{array}$ & $\begin{array}{c}-0.099 * * * \\
(0.021)\end{array}$ & $\begin{array}{c}-0.142^{* * *} \\
(0.036)\end{array}$ \\
\hline$\Delta \ln e r^{*} \times d$ & $\begin{array}{c}0.017 \\
(0.011)\end{array}$ & $\begin{array}{c}0.118^{* * * *} \\
(0.028)\end{array}$ & $\begin{array}{c}0.108^{* * * *} \\
(0.036)\end{array}$ & $\begin{array}{c}0.071 \\
(0.043)\end{array}$ & $\begin{array}{c}-0.103^{*} \\
(0.053)\end{array}$ & $\begin{array}{c}-0.200^{* * *} \\
(0.063)\end{array}$ & $\begin{array}{c}-0.289^{* * *} \\
(0.060)\end{array}$ \\
\hline$\Delta \ln e r^{*} \times$ Large & $\begin{array}{c}0.013 \\
(0.012)\end{array}$ & $\begin{array}{l}0.048^{*} \\
(0.025)\end{array}$ & $\begin{array}{c}0.075^{* *} \\
(0.032)\end{array}$ & $\begin{array}{c}0.075^{* *} \\
(0.035)\end{array}$ & $\begin{array}{c}0.120^{* * *} \\
(0.042)\end{array}$ & $\begin{array}{c}0.090^{* *} \\
(0.040)\end{array}$ & $\begin{array}{c}0.081^{* *} \\
(0.033)\end{array}$ \\
\hline$\Delta \ln e r^{*} \times d \times$ Large & $\begin{array}{c}0.003 \\
(0.018)\end{array}$ & $\begin{array}{l}-0.041 \\
(0.035)\end{array}$ & $\begin{array}{c}-0.081^{*} \\
(0.044)\end{array}$ & $\begin{array}{l}-0.058 \\
(0.043)\end{array}$ & $\begin{array}{c}-0.121^{* *} \\
(0.048)\end{array}$ & $\begin{array}{l}-0.048 \\
(0.046)\end{array}$ & $\begin{array}{l}-0.005 \\
(0.034)\end{array}$ \\
\hline $\begin{array}{l}\Delta \ln p \\
\quad \operatorname{lag} 1\end{array}$ & $-0.131^{* * *}$ & $-0.290 * * *$ & $-0.359 * * *$ & $-0.477 * * *$ & $-0.584^{* * *}$ & $-0.694^{* * *}$ & $-0.735^{* * *}$ \\
\hline $\operatorname{lag} 2$ & $\begin{array}{c}-0.126^{* * *} \\
(0.012)\end{array}$ & $\begin{array}{c}-0.212^{* * *} \\
(0.017)\end{array}$ & $\begin{array}{c}-0.331^{* * *} \\
(0.020)\end{array}$ & $\begin{array}{c}-0.436^{* * *} \\
(0.020)\end{array}$ & $\begin{array}{c}-0.562^{* * *} \\
(0.023)\end{array}$ & $\begin{array}{c}-0.617^{* * *} \\
(0.028)\end{array}$ & $\begin{array}{c}-0.702^{* * *} \\
(0.029)\end{array}$ \\
\hline $\operatorname{lag} 3$ & $\begin{array}{c}-0.074^{* * *} \\
(0.008)\end{array}$ & $\begin{array}{c}-0.158^{* * *} \\
(0.015)\end{array}$ & $\begin{array}{c}-0.272^{* * *} \\
(0.017)\end{array}$ & $\begin{array}{c}-0.390^{* * *} \\
(0.019)\end{array}$ & $\begin{array}{c}-0.493^{* * *} \\
(0.024)\end{array}$ & $\begin{array}{c}-0.542^{* * *} \\
(0.027)\end{array}$ & $\begin{array}{c}-0.596^{* * *} \\
(0.033)\end{array}$ \\
\hline $\operatorname{lag} 4$ & $\begin{array}{c}-0.035^{* * *} \\
(0.009)\end{array}$ & $\begin{array}{c}-0.132 * * * \\
(0.015)\end{array}$ & $\begin{array}{c}-0.217 * * * \\
(0.016)\end{array}$ & $\begin{array}{c}-0.324 * * * \\
(0.019)\end{array}$ & $\begin{array}{c}-0.408 * * * \\
(0.025)\end{array}$ & $\begin{array}{c}-0.503 * * * \\
(0.029)\end{array}$ & $\begin{array}{c}-0.561^{* * *} \\
(0.034)\end{array}$ \\
\hline $\operatorname{lag} 5$ & $\begin{array}{c}-0.045^{* * *} \\
(0.009)\end{array}$ & $\begin{array}{c}-0.124^{* * *} \\
(0.012)\end{array}$ & $\begin{array}{c}-0.210^{* * *} \\
(0.016)\end{array}$ & $\begin{array}{c}-0.289^{* * *} \\
(0.020)\end{array}$ & $\begin{array}{c}-0.365^{* * *} \\
(0.025)\end{array}$ & $\begin{array}{c}-0.410^{* * *} \\
(0.030)\end{array}$ & $\begin{array}{c}-0.460^{* * *} \\
(0.033)\end{array}$ \\
\hline$\Delta \ln q$ & & & & & & & \\
\hline $\operatorname{lag} 1$ & & $\begin{array}{c}0.004^{* * *} \\
(0.001)\end{array}$ & $\begin{array}{c}0.003^{* *} \\
(0.001)\end{array}$ & $\begin{array}{c}0.002 \\
(0.002)\end{array}$ & $\begin{array}{c}0.001 \\
(0.002)\end{array}$ & $\begin{array}{c}0.007^{* * *} \\
(0.002)\end{array}$ & $\begin{array}{c}0.008^{* * *} \\
(0.003)\end{array}$ \\
\hline lag 2 & $\begin{array}{c}0.003^{* * *} \\
(0.001)\end{array}$ & $\begin{array}{c}0.003^{* *} \\
(0.001)\end{array}$ & $\begin{array}{c}0.002 \\
(0.001)\end{array}$ & $\begin{array}{c}0.000 \\
(0.002)\end{array}$ & $\begin{array}{c}0.001 \\
(0.002)\end{array}$ & $\begin{array}{c}0.006^{* *} \\
(0.003)\end{array}$ & $\begin{array}{c}0.008 * * * \\
(0.003)\end{array}$ \\
\hline $\operatorname{lag} 3$ & $\begin{array}{c}0.001 \\
(0.001)\end{array}$ & $\begin{array}{c}0.001 \\
(0.001)\end{array}$ & $\begin{array}{l}-0.000 \\
(0.002)\end{array}$ & $\begin{array}{c}-0.002 \\
(0.002)\end{array}$ & $\begin{array}{c}0.000 \\
(0.002)\end{array}$ & $\begin{array}{c}0.004 \\
(0.003)\end{array}$ & $\begin{array}{c}0.008^{* *} \\
(0.003)\end{array}$ \\
\hline $\operatorname{lag} 4$ & $\begin{array}{c}0.000 \\
(0.001)\end{array}$ & $\begin{array}{c}0.001 \\
(0.001)\end{array}$ & $\begin{array}{c}-0.001 \\
(0.002)\end{array}$ & $\begin{array}{l}-0.000 \\
(0.002)\end{array}$ & $\begin{array}{l}-0.000 \\
(0.003)\end{array}$ & $\begin{array}{c}0.004 \\
(0.003)\end{array}$ & $\begin{array}{c}0.006^{*} \\
(0.003)\end{array}$ \\
\hline $\operatorname{lag} 5$ & $\begin{array}{c}0.000 \\
(0.001)\end{array}$ & $\begin{array}{l}-0.001 \\
(0.001)\end{array}$ & $\begin{array}{c}-0.003^{*} \\
(0.001)\end{array}$ & $\begin{array}{c}0.001 \\
(0.002)\end{array}$ & $\begin{array}{c}0.000 \\
(0.002)\end{array}$ & $\begin{array}{l}0.004^{*} \\
(0.003)\end{array}$ & $\begin{array}{c}0.002 \\
(0.002)\end{array}$ \\
\hline IIICI & & & & & & & \\
\hline $\operatorname{lag} 1$ & $\begin{array}{c}0.654^{* * *} \\
(0.044)\end{array}$ & $\begin{array}{c}0.883^{* * *} \\
(0.063)\end{array}$ & $\begin{array}{c}1.473^{* * *} \\
(0.091)\end{array}$ & $\begin{array}{c}0.917^{* * *} \\
(0.106)\end{array}$ & $\begin{array}{c}0.634^{* * *} \\
(0.150)\end{array}$ & $\begin{array}{l}-0.221 \\
(0.160)\end{array}$ & $\begin{array}{c}-0.384^{* *} \\
(0.163)\end{array}$ \\
\hline lag 2 & $\begin{array}{c}-0.327^{* * *} \\
(0.054)\end{array}$ & $\begin{array}{c}0.383^{* * *} \\
(0.061)\end{array}$ & $\begin{array}{c}0.033 \\
(0.072)\end{array}$ & $\begin{array}{c}-0.410^{* * * *} \\
(0.084)\end{array}$ & $\begin{array}{c}-0.648^{* * * *} \\
(0.111)\end{array}$ & $\begin{array}{c}-0.589^{* * * *} \\
(0.121)\end{array}$ & $\begin{array}{c}0.516^{* * *} \\
(0.116)\end{array}$ \\
\hline lag 3 & $\begin{array}{c}0.632^{* * *} \\
(0.060)\end{array}$ & $\begin{array}{c}0.590^{* * *} \\
(0.057)\end{array}$ & $\begin{array}{c}0.225^{* * *} \\
(0.077)\end{array}$ & $\begin{array}{c}-0.169^{* *} \\
(0.084)\end{array}$ & $\begin{array}{c}-0.663^{* * *} \\
(0.105)\end{array}$ & $\begin{array}{l}-0.075 \\
(0.093)\end{array}$ & $\begin{array}{c}0.310 \\
(0.294)\end{array}$ \\
\hline $\operatorname{lag} 4$ & $\begin{array}{l}-0.068 \\
(0.049)\end{array}$ & $\begin{array}{l}-0.106 \\
(0.065)\end{array}$ & $\begin{array}{c}-0.610^{* * * *} \\
(0.081)\end{array}$ & $\begin{array}{c}-0.466^{* * * *} \\
(0.098)\end{array}$ & $\begin{array}{c}0.348^{* * *} \\
(0.110)\end{array}$ & $\begin{array}{c}1.275^{* * *} \\
(0.117)\end{array}$ & $\begin{array}{c}-1.727^{* * *} \\
(0.548)\end{array}$ \\
\hline $\operatorname{lag} 5$ & $\begin{array}{c}0.290^{* * * *} \\
(0.036)\end{array}$ & $\begin{array}{c}0.026 \\
(0.059)\end{array}$ & $\begin{array}{c}0.095 \\
(0.069)\end{array}$ & $\begin{array}{l}-0.106 \\
(0.086)\end{array}$ & $\begin{array}{l}-0.089 \\
(0.112)\end{array}$ & $\begin{array}{c}-3.001^{* * * *} \\
(0.455)\end{array}$ & $\begin{array}{c}-2.767^{* * * *} \\
(0.512)\end{array}$ \\
\hline $\begin{array}{l}\text { Fixed effects } \\
\text { Item }\end{array}$ & Yes & Yes & Yes & Yes & Yes & Yes & Yes \\
\hline $\begin{array}{l}R \text {-squared } \\
\text { Observations }\end{array}$ & $\begin{array}{c}0.066 \\
45,507\end{array}$ & $\begin{array}{c}0.140 \\
42,473\end{array}$ & $\begin{array}{c}0.193 \\
39,439\end{array}$ & $\begin{array}{c}0.239 \\
36,405\end{array}$ & $\begin{array}{c}0.263 \\
33,371\end{array}$ & $\begin{array}{c}0.283 \\
30,337\end{array}$ & $\begin{array}{c}0.311 \\
27,303\end{array}$ \\
\hline
\end{tabular}

Note: $\overline{\bar{T}}$ The table presents the full estimation results of model (5) using the sales-weighted exchange rate,$e r^{*}$, for $h=0,2, \cdots, 10,12$ and $L=5$. Robust standard errors (clustered by product) are in parentheses. The notation ${ }^{* * *}$ indicates significance at $1 \%$ level, ${ }^{* *}$ at $5 \%$ level, ${ }^{*}$ at $10 \%$ level. 
Figure A1: Average unit price trend from unbalanced panel

Beer

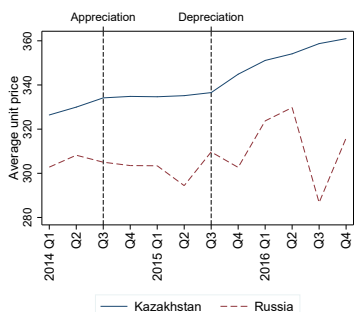

Draught beer

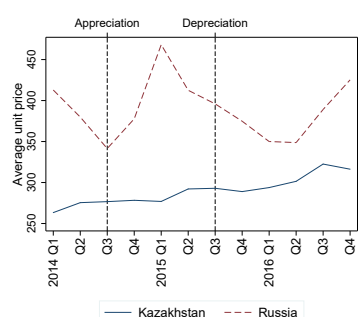

Fresh refrigerated

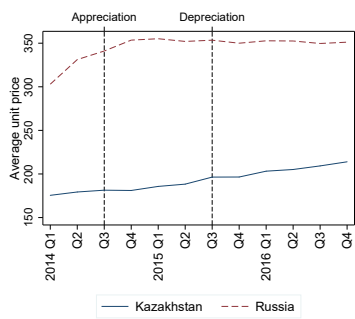

Liquid milk products

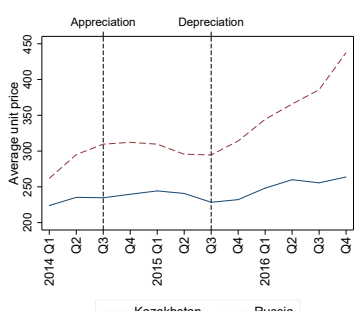

Cheese curds

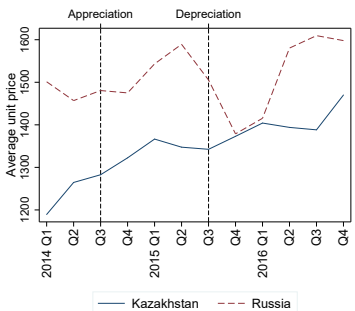

Drink yogurt refrigerate

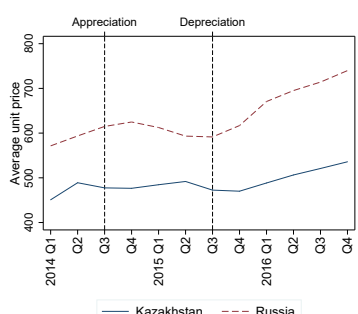

Fruit \& vegetable juice

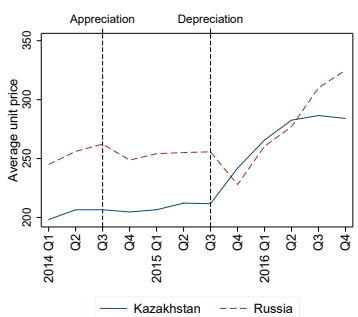

Mineral water

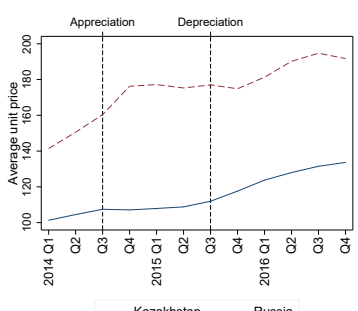

Cultured milk

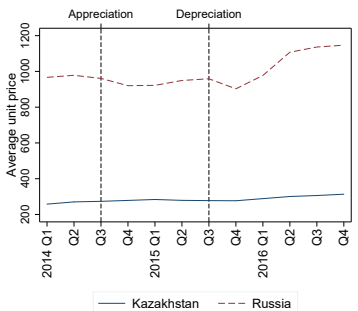

Energy drink

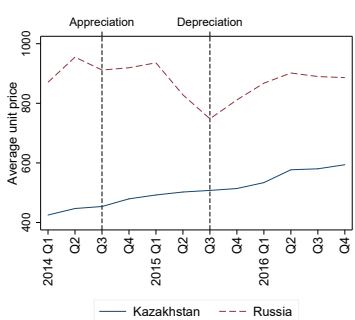

Glazed curds

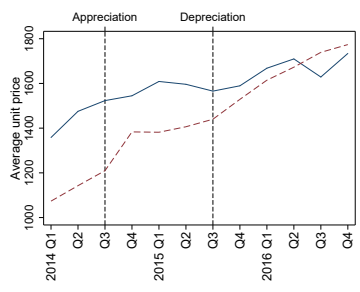

- Kazakstan --- Russia

Noodles extra flavouring

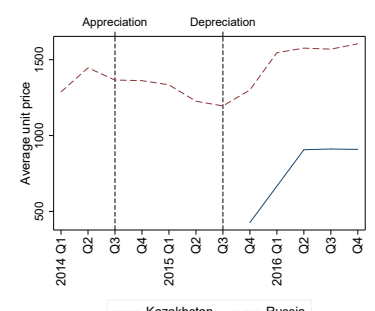

Dessert \& pudding

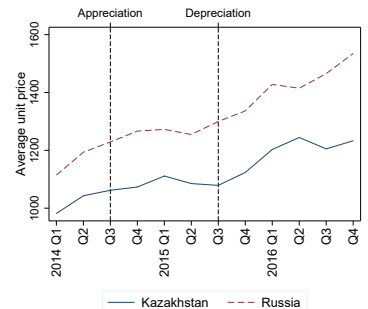

Evaporated milk

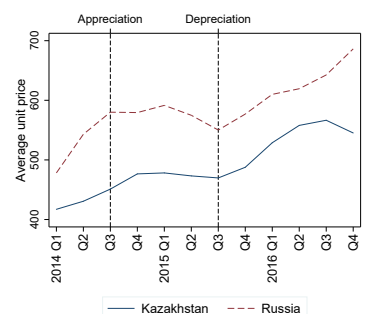

Juice with milk additive

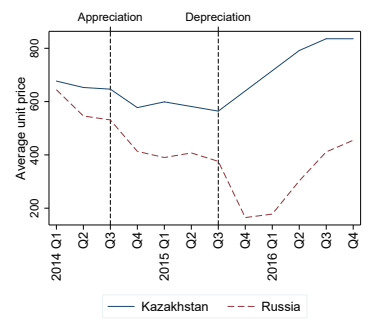

Pasta dry

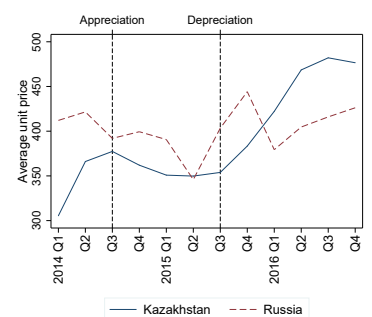

Soft drinks

Sour cream

Yogurt spoonable
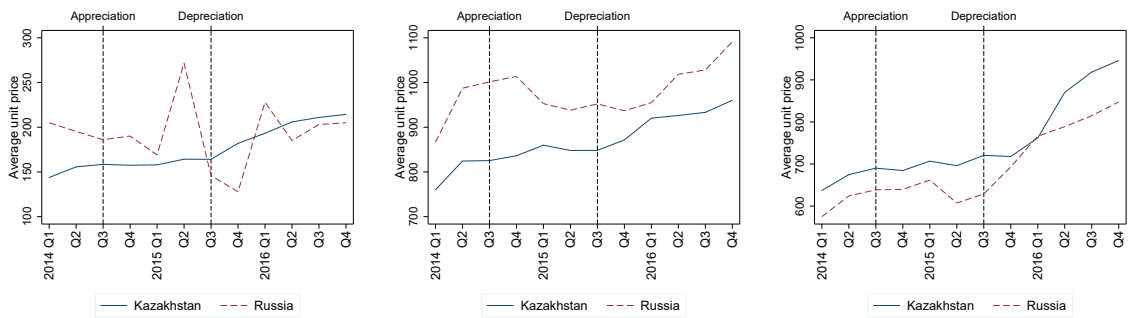

Note: Each panel of the figure compares the average unit price of domestic and Russian products in a product category. Unbalanced panel is used to draw the price trends. 
Figure A2: Average unit price trend from balanced panel

Beer

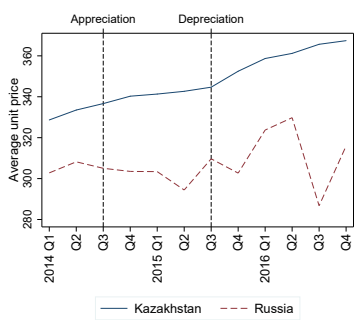

Draught beer

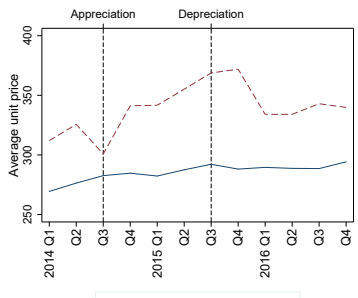

Fresh refrigerated

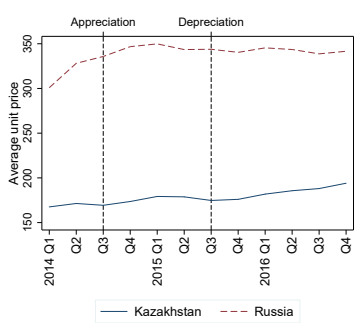

Mineral water

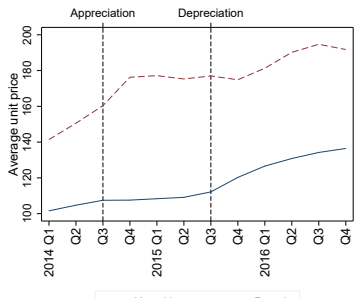

Cheese curds

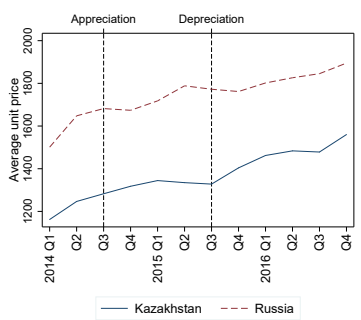

Drink yogurt refrigerate

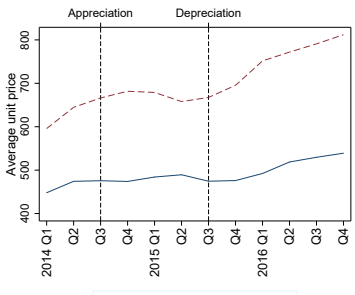

— Kazakhstan --- Russia

Fruit \& vegetable juice

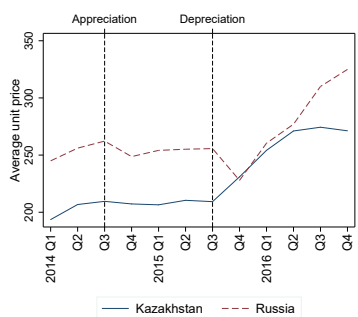

Pasta dry

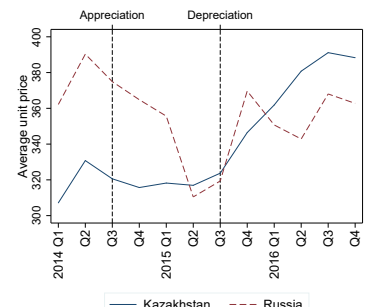

Cultured milk

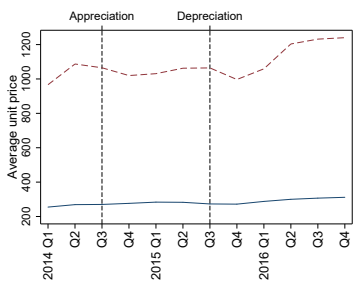

- Kazakhstan --- Russ

Energy drink

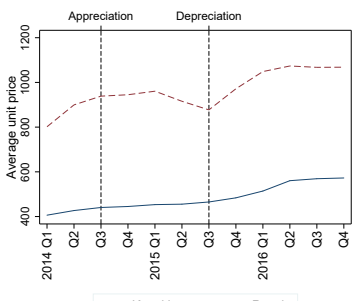

Glazed curds

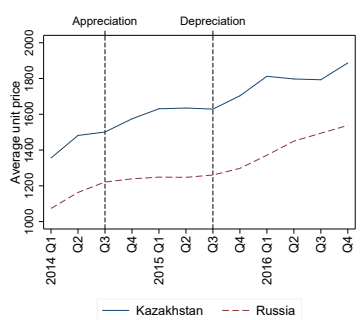

Sour cream

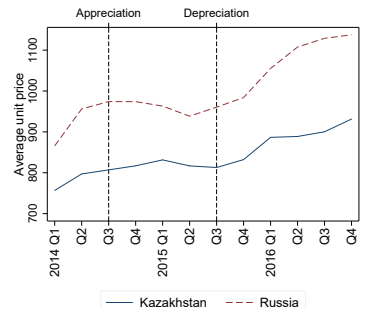

Dessert \& pudding

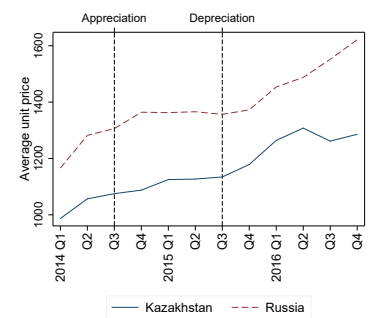

Evaporated milk

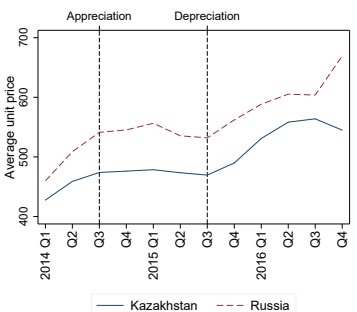

Liquid milk products

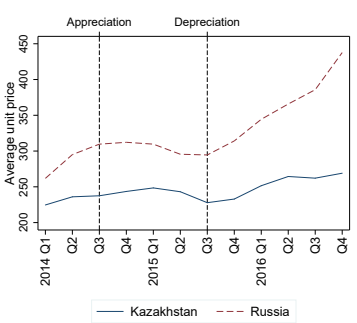

Yogurt spoonable

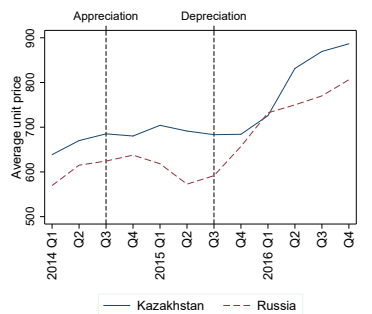

Note: Each panel of the figure compares the average unit price of domestic and Russian products in a product category. Balanced panel is used to draw the price trends. 
Figure A3: Monthly change in the effective exchange rate

Beer

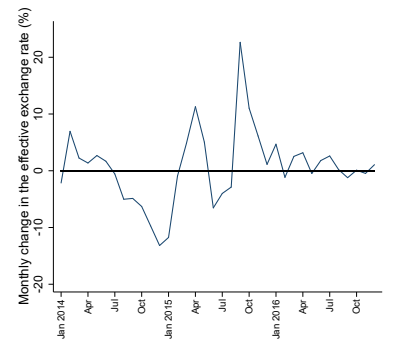

Dessert \& pudding

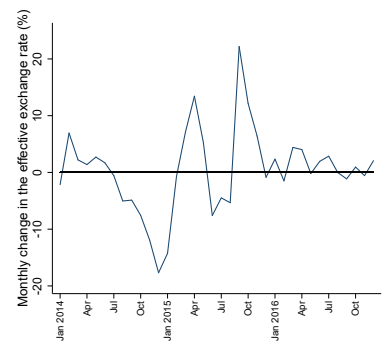

Evaporated milk

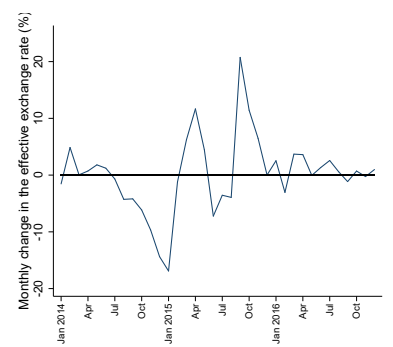

Juice with milk additive

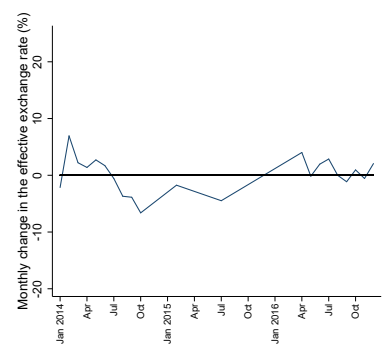

Pasta dry

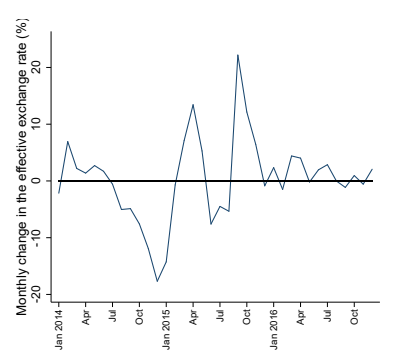

Cheese curds

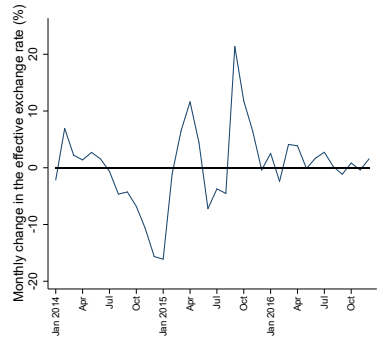

Draught beer

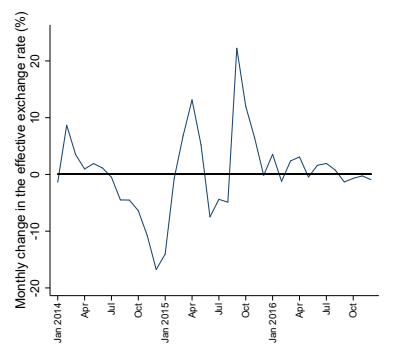

Fresh refrigerated

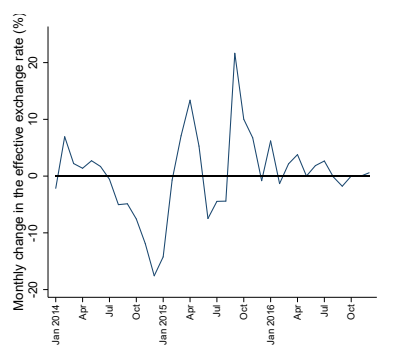

Liquid milk products

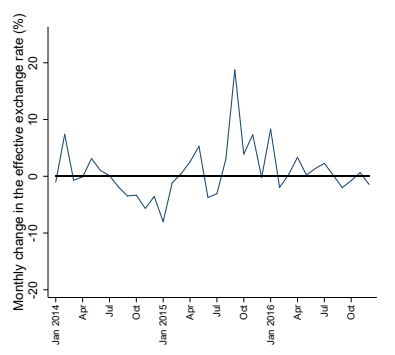

Soft drinks

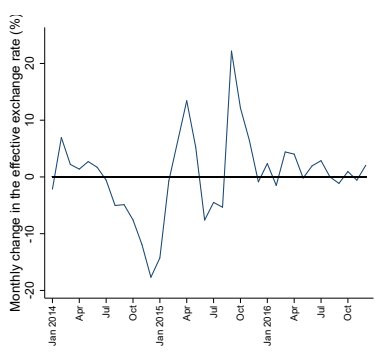

Cigarette

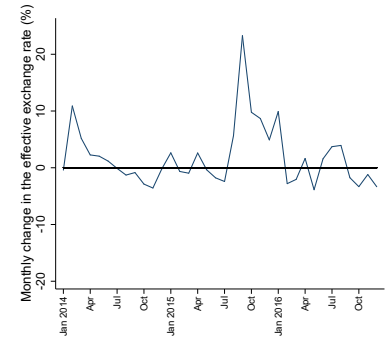

Drink yogurt refrigerate

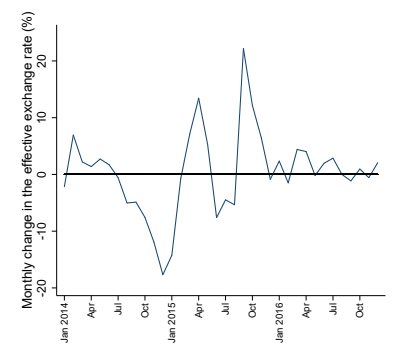

Fruit \& vegetable juice

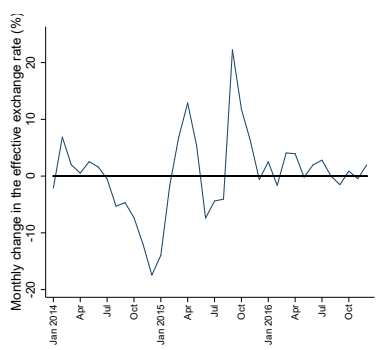

Mineral water

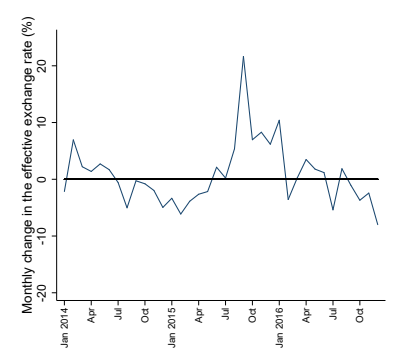

Sour cream

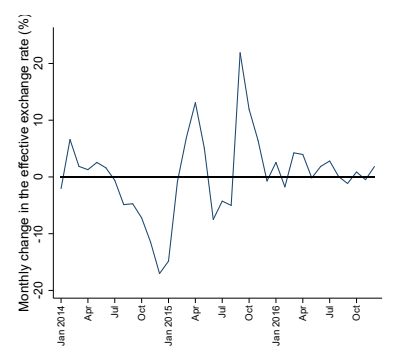

Cultured milk

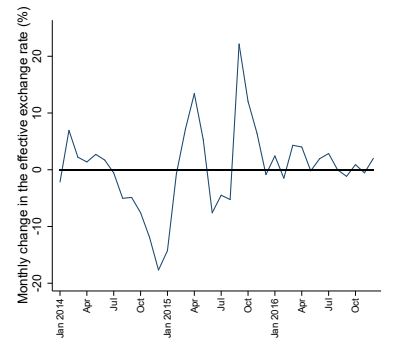

Energy drink

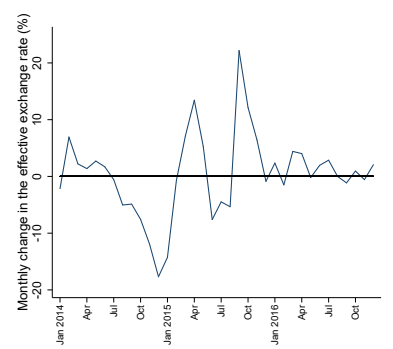

Glazed curds

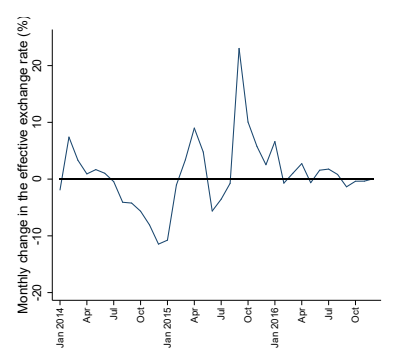

Noodles dry with extra flavouring

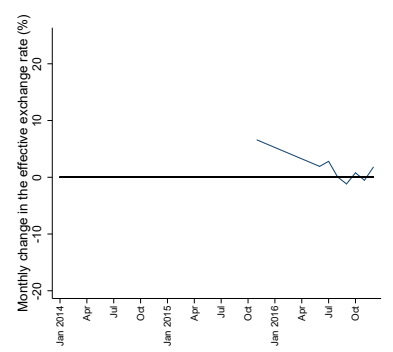

Yogurt spoonable

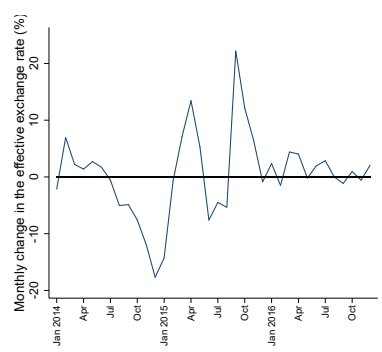

Note: Each panel of the Figure presents the monthly change in the sales-weighted exchange rate in each of the 20 product category. 
Figure A4: ERPT with $L=4$ and 6

Panel A: Imported products

$$
L=4
$$

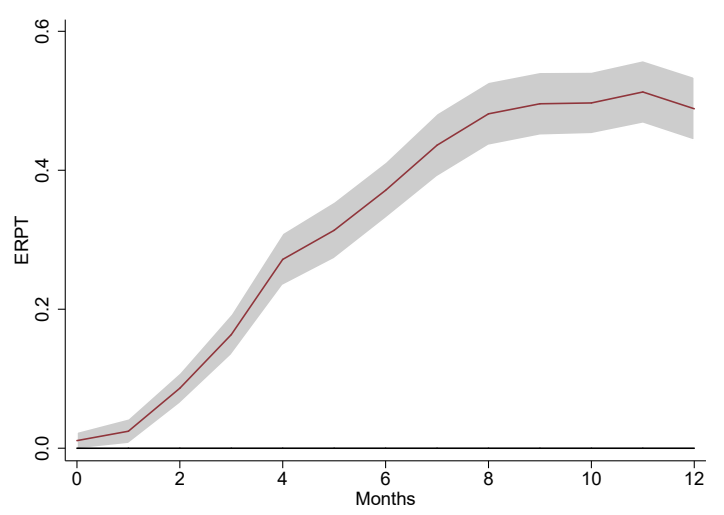

$$
L=6
$$

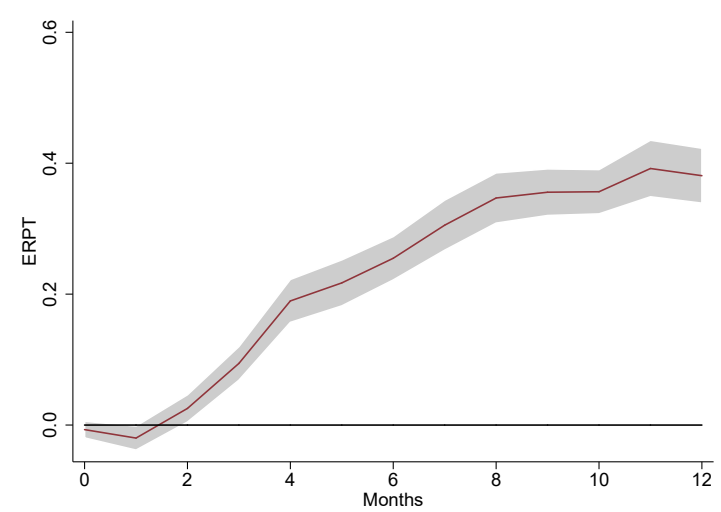

Panel B: Domestic products

$$
L=4
$$

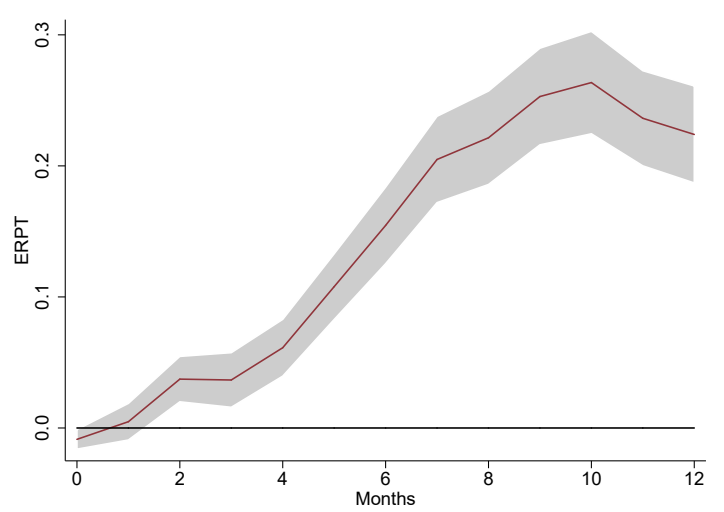

$L=6$

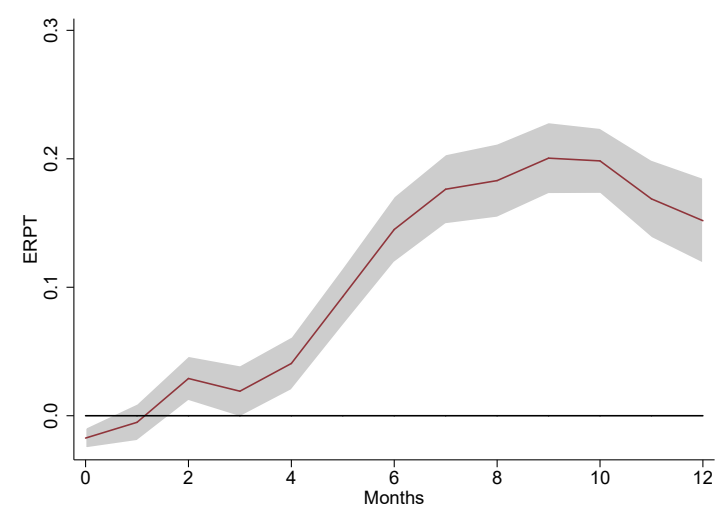

Note: The two panels of the figure graphically present the estimates of the ERPTs for imported and domestic products up to $h=12$, that is, up to one year after the shock, for $L=4$ and 6 along with 95 percent confidence bands. 
Figure A5: Asymmetric ERPT with $L=4$ and 6

Panel A: Imported products

$$
L=4
$$

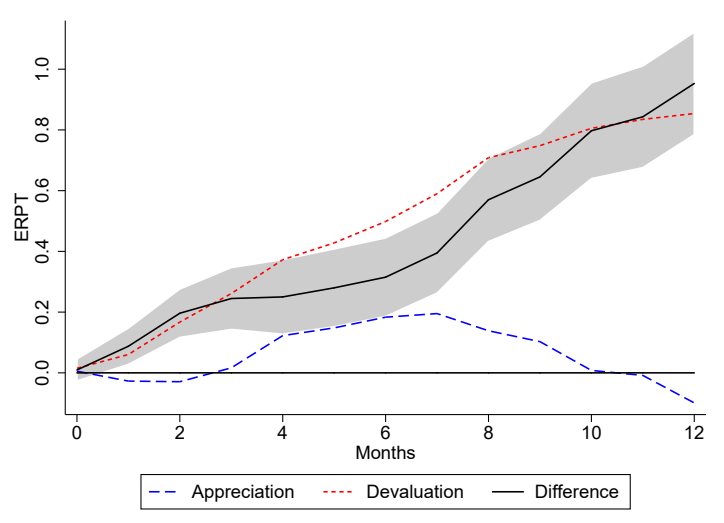

$$
L=6
$$

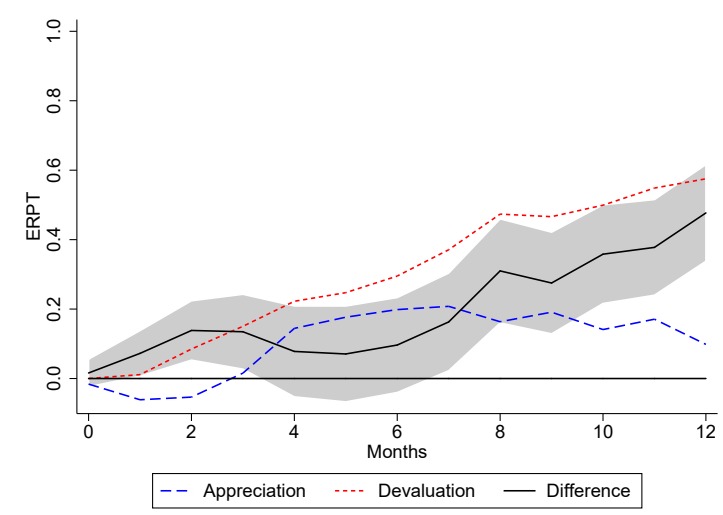

Panel B: Domestic products

$$
L=4
$$

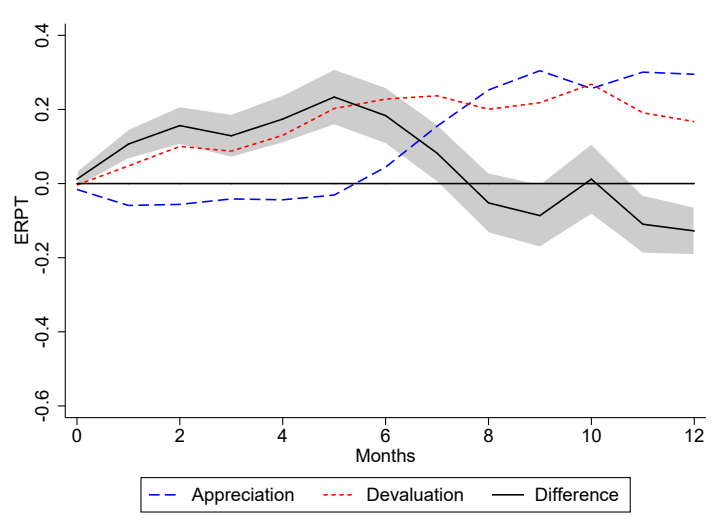

$$
L=6
$$

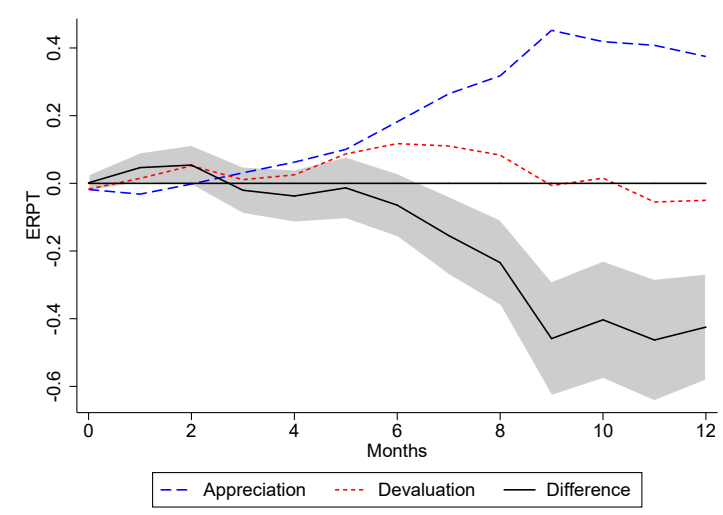

Note: The two panels of the figure present the estimated cumulative price responses under depreciation and appreciation when $L=4$ and 6 , and the gap between them along with 95 percent confidence bands. 
Figure A6: ERPT and the retailer size with $L=4$ and 6

Panel A: Imported products

$$
L=4
$$

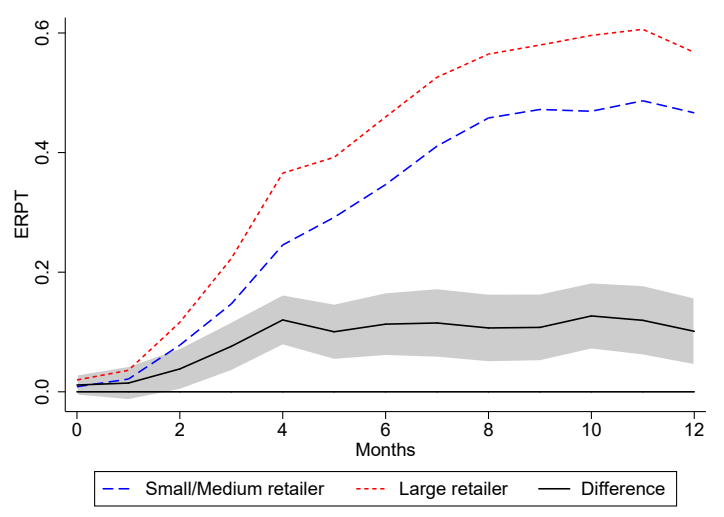

$$
L=6
$$

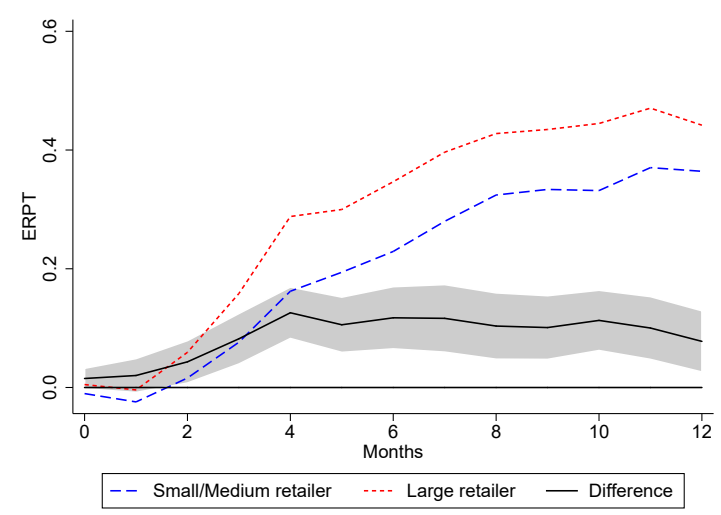

Panel B: Domestic products

$$
L=4
$$

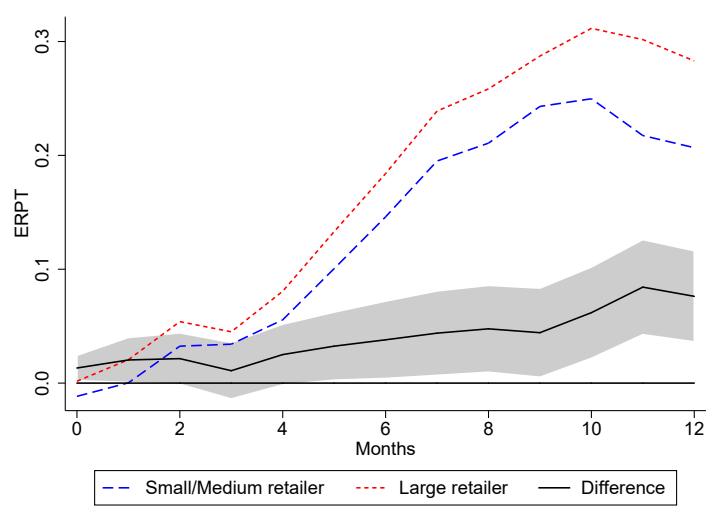

$$
L=6
$$

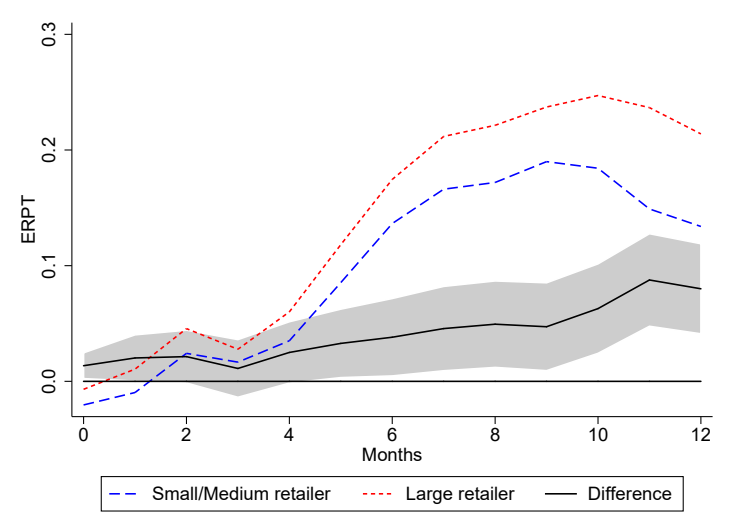

Note: The two panels of the figure present the estimates of the ERPTs at large and small/medium retailers, and the gap between them up to $h=12$ for $L=4$ and 6 along with 95 percent confidence bands. 
Figure A7: Asymmetric ERPT and the retailer size with $L=4$ and 6

Panel A: Imported products

$$
L=4
$$

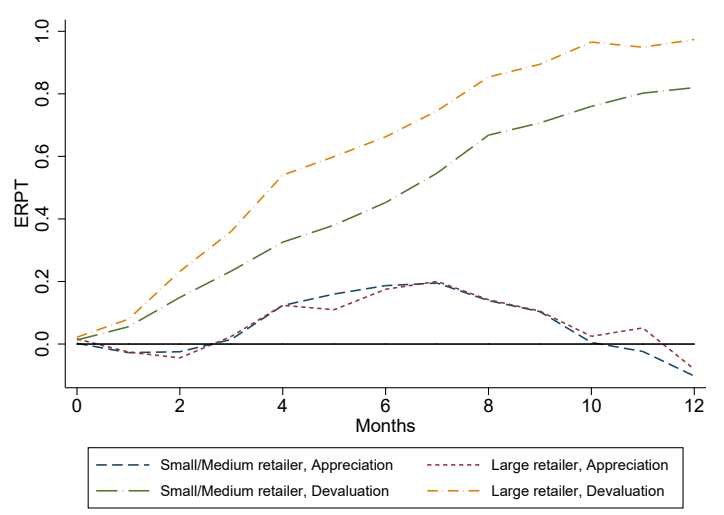

$L=6$

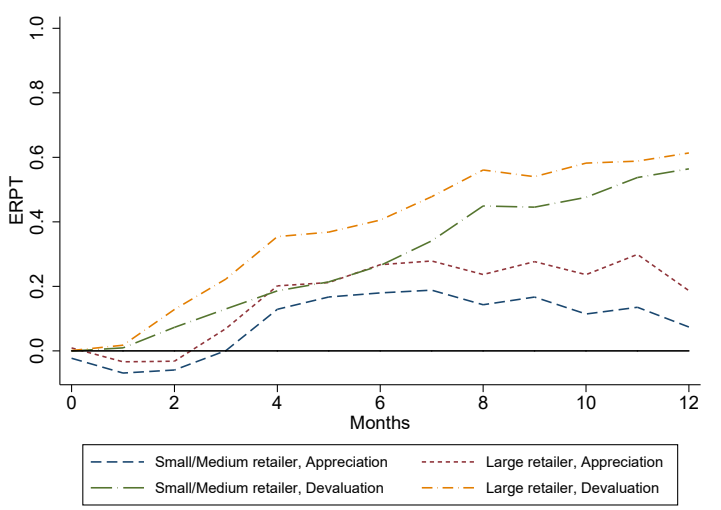

Panel B: Domestic products

$L=4$

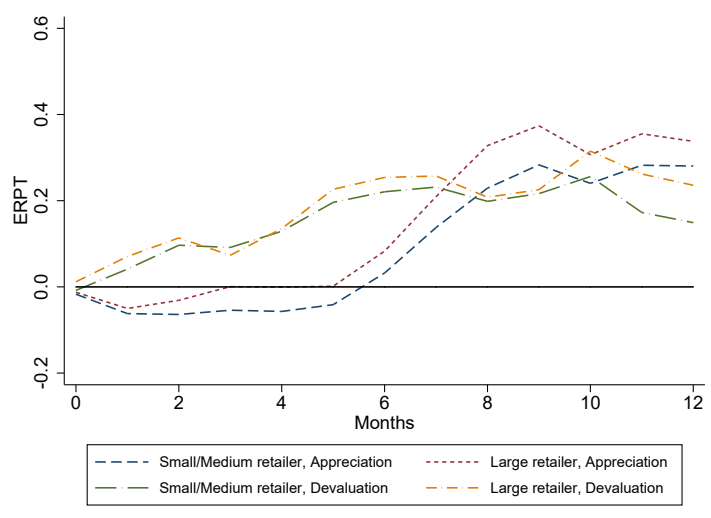

$L=6$

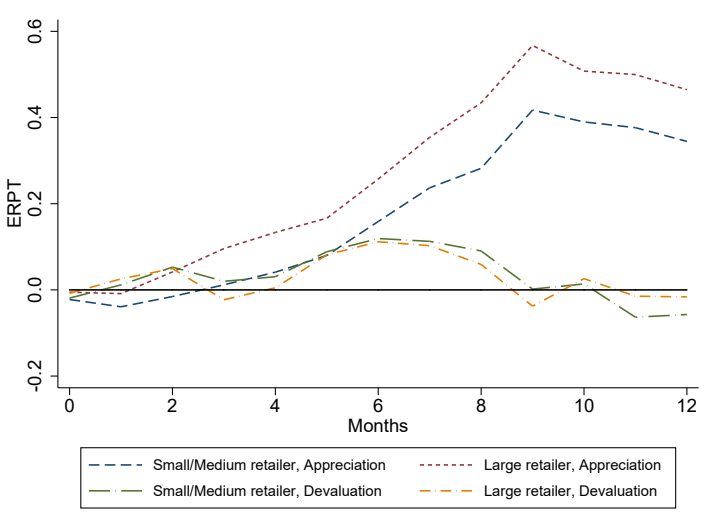

Note: The two panels of the figure present the estimates of the asymmetric ERPTs when $L=4$ and 6 at large and small/medium retailers. 
Figure A8: Estimating ERPTs for imported products with Driscoll and Kraay standard errors Panel A: ERPTs

$$
L=4
$$

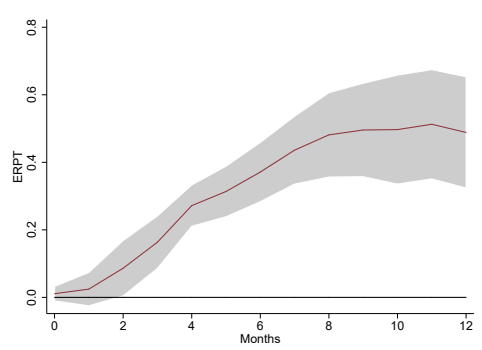

$$
L=4
$$

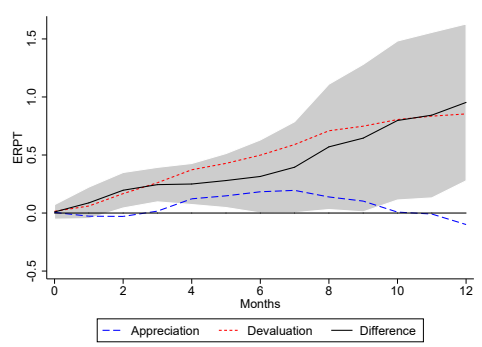

$L=5$

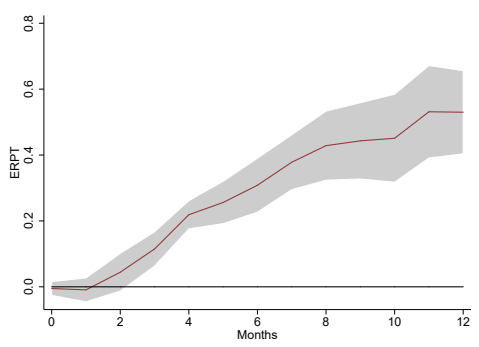

Panel B: Asymmetric ERPTs

$$
L=5
$$

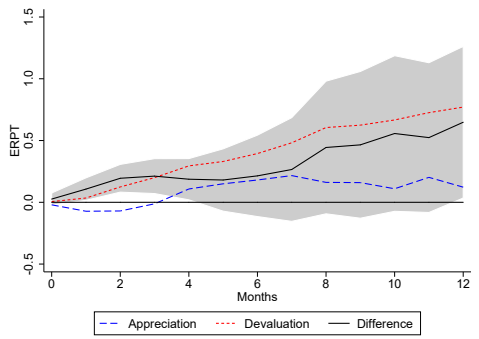

Panel C: ERPT and the retailer size

$$
L=4
$$

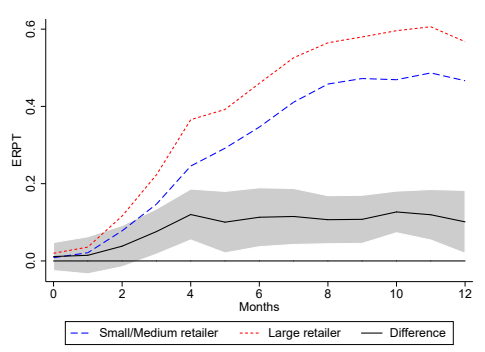

$L=5$

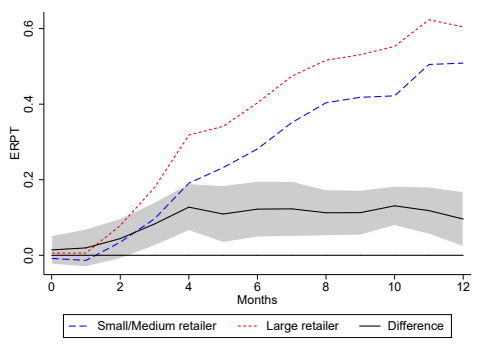

$L=6$

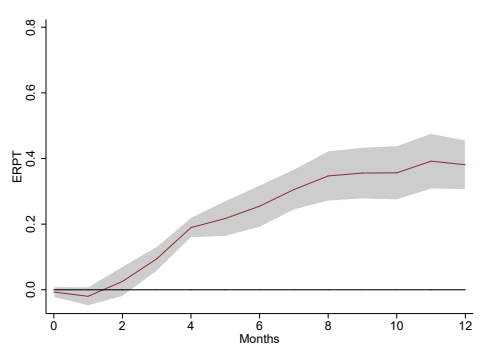

$$
L=6
$$
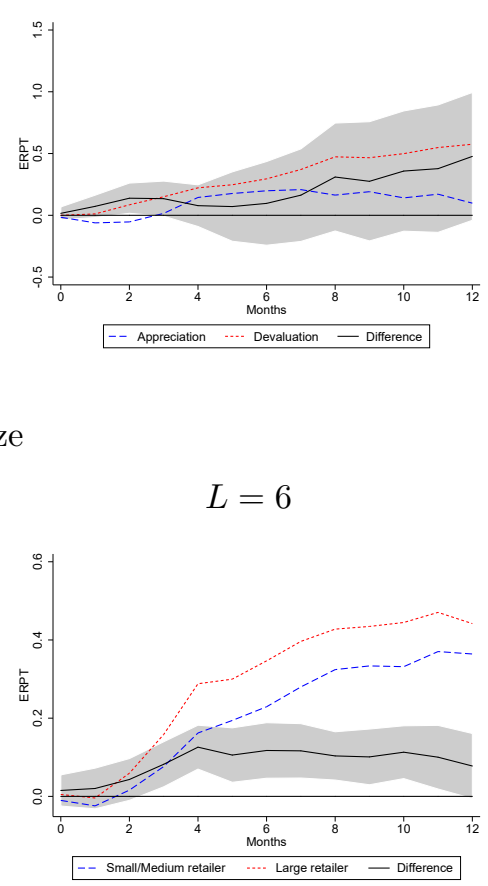

Note: The figure presents the estimates of the ERPTs for imported products along with 95 percent confidence bands that are derived using Driscoll and Kraay (1998)'s nonparametric covariance matrix estimator. 
Figure A9: Estimating ERPTs for domestic products with Driscoll and Kraay standard errors Panel A: ERPTs

$$
L=4
$$

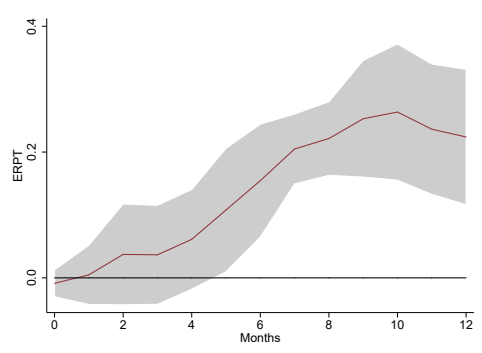

$$
L=4
$$

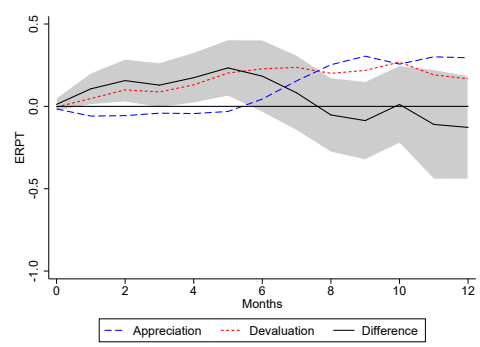

$L=5$

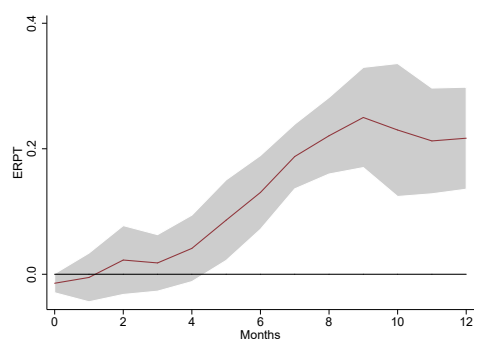

Panel B: Asymmetric ERPTs

$L=5$

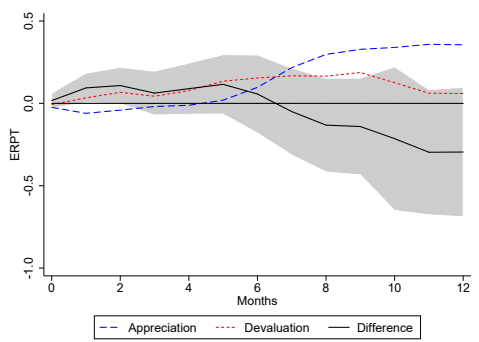

$L=6$

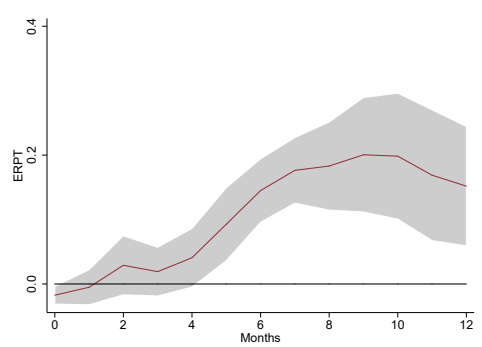

$$
L=6
$$

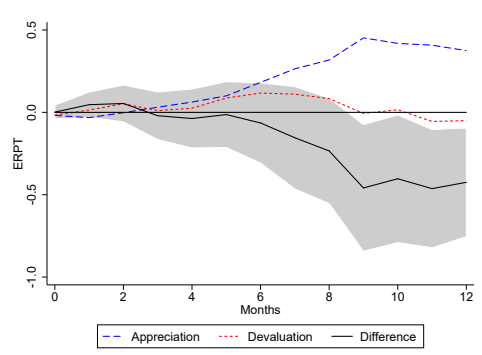

Panel C: ERPT and the retailer size

$$
L=4
$$

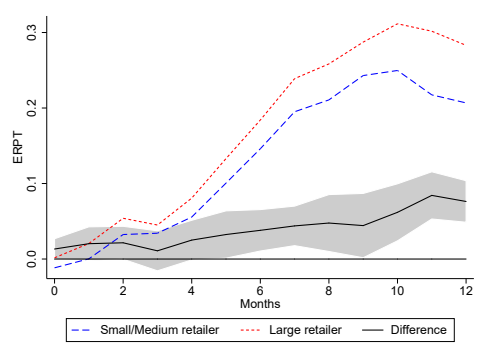

$L=5$

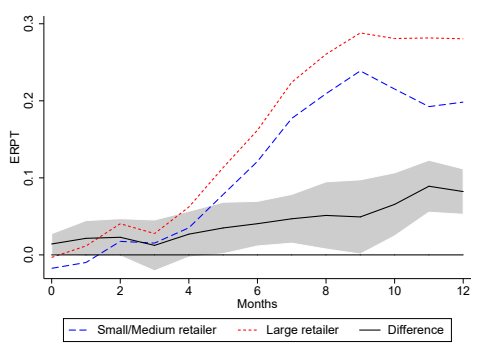

$L=6$

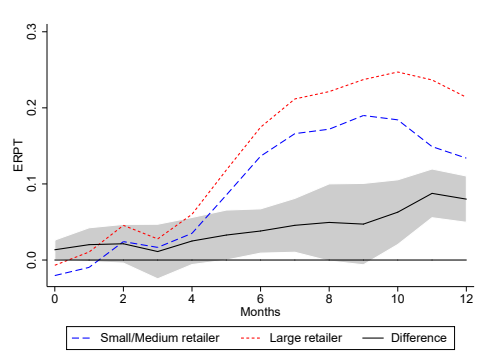

Note: The figure presents the estimates of the ERPTs for domestic products along with 95 percent confidence bands that are derived using Driscoll and Kraay (1998)'s nonparametric covariance matrix estimator. 
Figure A10: Estimating ERPTs for imported products with unbalanced panel Panel A: ERPTs
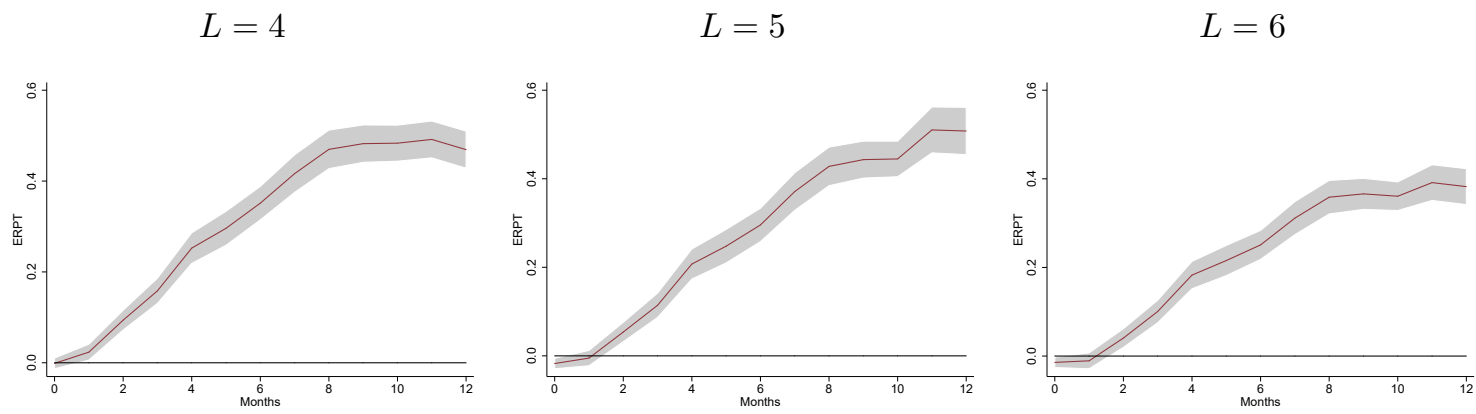

Panel B: Asymmetric ERPTs

$$
L=4
$$
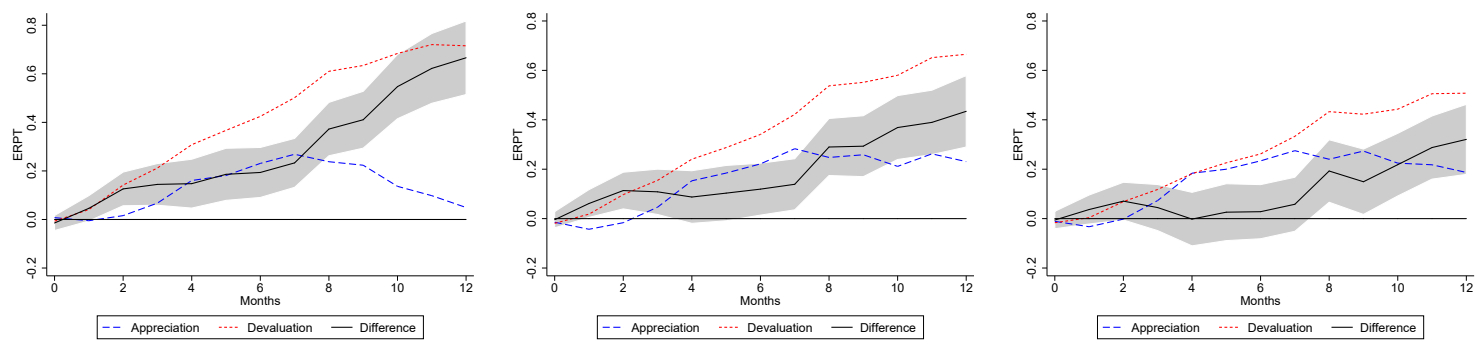

Panel C: ERPT and the retailer size

$$
L=4
$$

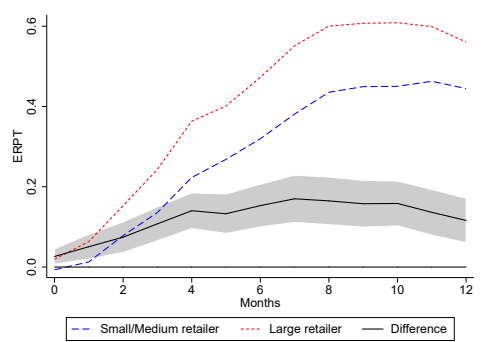

$L=5$

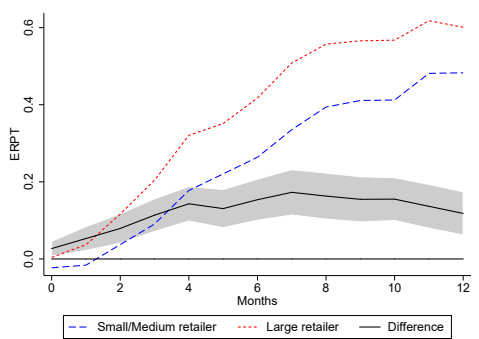

$L=6$

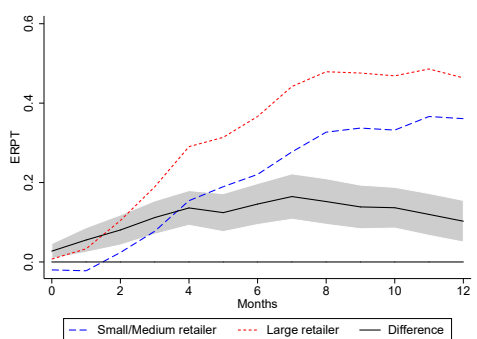

Note: The figure presents the estimates of the ERPTs for imported products along with 95 percent confidence bands using unbalanced panel. 
Figure A11: Estimating ERPTs for domestic products with unbalanced panel Panel A: ERPTs
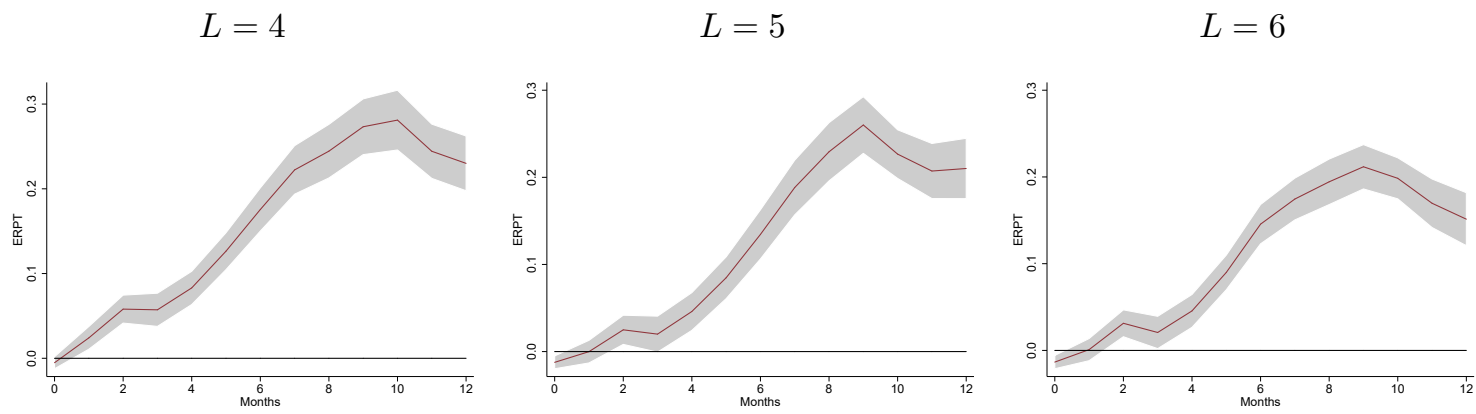

Panel B: Asymmetric ERPTs

$$
L=4
$$
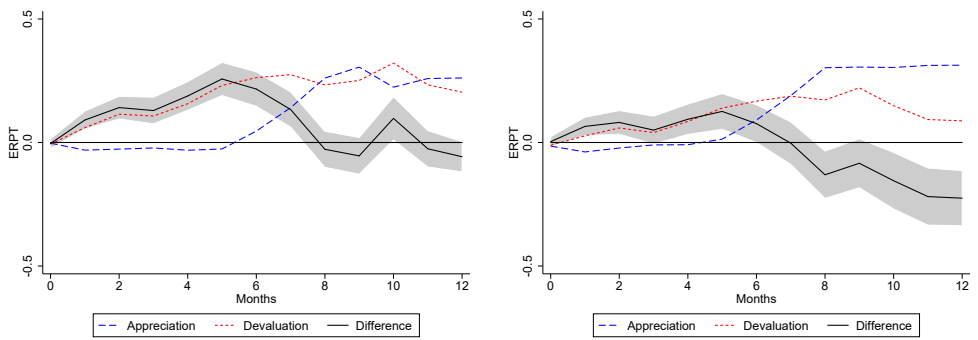

- - Appreciation $\cdots$ Devaluation - Difference
$L=6$

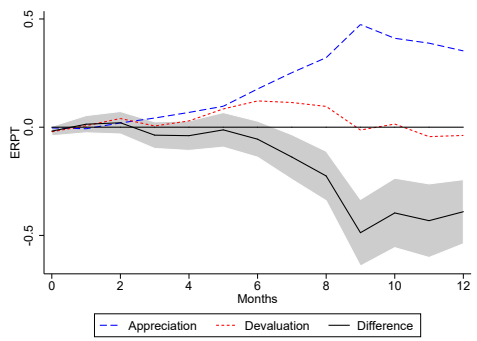

Panel C: ERPT and the retailer size

$$
L=4
$$

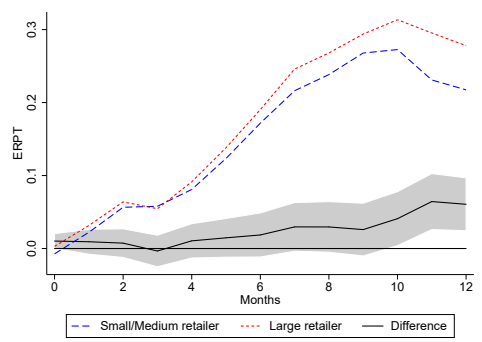

$L=5$

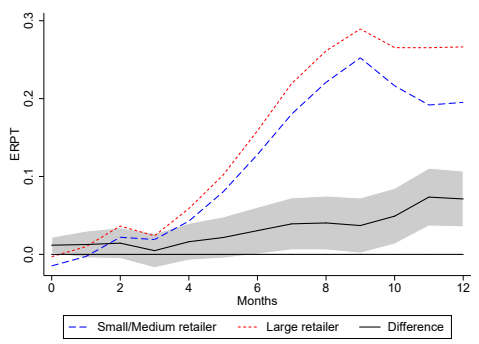

$L=6$

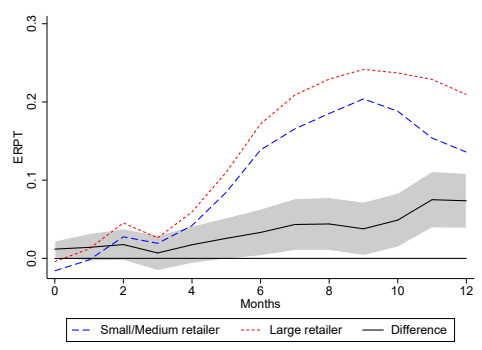

Note: The figure presents the estimates of the ERPTs for domestic products along with 95 percent confidence bands using unbalanced panel. 


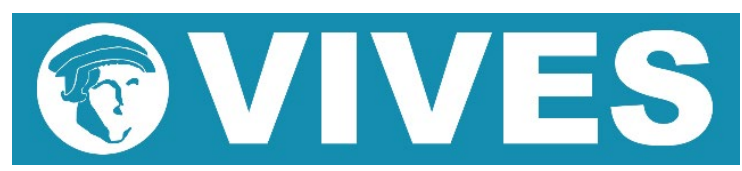

Research Center for Regional Economics

Vlamingenstraat 83 - Box 03550

3000 Leuven, Belgium

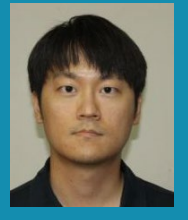

\section{Kim In Kyung}

Nazarbayev University, Department of Economics

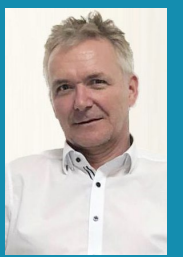

Jozef Konings

VIVES (KU Leuven)

Nazarbayev University

University of Liverpool Management School

CEPR

jozef.konings@kuleuven.be

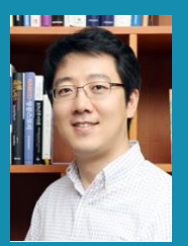

\section{Lee Jinhyuk}

Korea University, Department of Economics

Copyright $\odot 2021$

Discussion papers are in draft form. This discussion paper is distributed for purposes of comment and discussion only. It may not be reproduced without permission of the copyright holder.

An electronic version of the discussion paper is available on the VIVES website:

http://www.feb.kuleuven.be/VIVES 